\title{
Processing of novelty and familiarity in the aging brain
}

Citation for published version (APA):

Tóth, M. (2022). Processing of novelty and familiarity in the aging brain. [Doctoral Thesis, Maastricht University]. Maastricht University. https://doi.org/10.26481/dis.20220201mt

Document status and date:

Published: 01/01/2022

DOI:

10.26481/dis.20220201mt

Document Version:

Publisher's PDF, also known as Version of record

\section{Please check the document version of this publication:}

- A submitted manuscript is the version of the article upon submission and before peer-review. There can be important differences between the submitted version and the official published version of record.

People interested in the research are advised to contact the author for the final version of the publication, or visit the DOI to the publisher's website.

- The final author version and the galley proof are versions of the publication after peer review.

- The final published version features the final layout of the paper including the volume, issue and page numbers.

Link to publication

\footnotetext{
General rights rights.

- You may freely distribute the URL identifying the publication in the public portal. please follow below link for the End User Agreement:

www.umlib.nl/taverne-license

Take down policy

If you believe that this document breaches copyright please contact us at:

repository@maastrichtuniversity.nl

providing details and we will investigate your claim.
}

Copyright and moral rights for the publications made accessible in the public portal are retained by the authors and/or other copyright owners and it is a condition of accessing publications that users recognise and abide by the legal requirements associated with these

- Users may download and print one copy of any publication from the public portal for the purpose of private study or research.

- You may not further distribute the material or use it for any profit-making activity or commercial gain

If the publication is distributed under the terms of Article $25 \mathrm{fa}$ of the Dutch Copyright Act, indicated by the "Taverne" license above, 


\title{
Processing of Novelty and Familiarity in the Aging Brain
}

\author{
Mónika Tóth
}

Department of Neuropsychology \& Psychopharmacology Faculty of Psychology and Neuroscience I Maastricht University 

Cover design: Manó Szentesi, Manodesign (manodesign@t-online.hu)

Printing: Gildeprint - The Netherlands

ISBN: 978-94-6419-409-8

Copyright: (C) 2021 Mónika Tóth

All rights reserved. No part of this publication may be reproduced or transmitted in any form or by any means, without written permission from the author or, when applicable, by the publisher from the published articles. The copyright of the published articles has been transferred to the respective journals.

Printing of this dissertation was kindly supported by Maastricht University. 



\title{
Processing of Novelty and Familiarity in the Aging Brain
}

\author{
DISSERTATION
}

To obtain the degree of doctor at Maastricht University, on the authority of Rector Magnificus, Prof. dr. Pamela Habibović, in accordance with the decision of the Board of Deans, to be defended in public on Tuesday 01 February 2022 at 13:00 hours

by

Mónika Tóth

born on the $25^{\text {th }}$ of November 1979, in Beregovo 


\section{Promotor}

Prof. dr. A. Blokland

\section{Co-promotor}

Dr. A. Sambeth

\section{Assessment Comitee}

Prof. dr. W. Riedel (chair)

Prof. dr. M. Meeter, Vrije Universiteit Amsterdam

Prof. dr. J.L. Kenemans, Utrecht University

Dr. C. Quaedflieg

The research described in this thesis was conducted at the Department of Neuropsychology and Psychopharmacology, Faculty of Psychology and Neuroscience, Maastricht University, The Netherlands. 


\section{Table of Contents}

I. Chapter: General Introduction and Outline 11

I.1. Background 12

I.1.1. Old/new Recognition 14

I.1.2. The Impact of the Stimulus on old/new Recognition 16

I.1.3. Modelling Age-related Memory Impairments with Biperiden 18

I.2. The Aim and Approach of the current Dissertation 19

I.3. Outline of the Dissertation 19

II. Chapter: The Role of pre-experimental Familiarity and Memory Strength in old/new Recognition 21

II.1. Abstract 22

II.2. Introduction 23

II.3. Methods 26

II.3.1. Participants 27

II.3.2. Procedure _ 27

II.3.3. Data Analysis_ 30

II.4. Results ___ 33

II.4.1. Visual Stimuli _ 34

II.4.2. Verbal Stimuli _ 36

II.5. Discussion _ 37

II.5.1. The Impact of Memory Strength __ 38

II.5.2. The Role of pre-experimental Familiarity___ 39

II.5.3. Response Times __ 41

II.6. Appendix: The Effect of Item Proportions: Control Experiment _ 44

II.6.1. Rationale _ 44

II.6.2. Methods 45

II.6.2.1. Participants _ 45

II.6.2.2. Procedure _ 45

II.6.3. Data Analyses — 46

II.6.4. Results 46

II.6.4.1. Abstract Figures _ 46

II.6.4.2. Non-words 49

II.6.5. Brief discussion of the results___ 49

III. Chapter: EEG Correlates of old/new Discrimination

Performance involving Abstract Figures and Non-Words 
III.2. Introduction ___ 55

III.3. Materials and Methods___ 60

III.3.1. Participants _ 60

III.3.2. Procedure 60

III.3.3. Data Analysis _ 64

III.4. Results ___ 67

III.4.1. Behavioral 67

III.4.1.1. Abstract Figures _ 68

III.4.1.2. Non-Words_ 70

III.4.2. EEG_ 71

III.4.2.1. Abstract Figures _ 71

III.4.2.2. Non-Words__ 74

III.5. Discussion 76

III.5.1. Abstract Figures__ 77

III.5.2. Non-Words 80

III.6. Conclusions __ 83

IV. Chapter: Age Effects on old/new Recognition Performance involving Abstract Figures and Non-Words__ 85

IV.1. Abstract ___ 86

IV.2. Introduction ___ 88

IV.3. Materials and Methods___ 92

IV.3.1. Participants _ 92

IV.3.2. Procedure 93

IV.3.3. Data Analysis _ 95

IV.4. Results ___ 98

IV.4.1. Abstract Figures_ 100

IV.4.2. Non-Words 102

IV.5. Discussion __ 103

IV.5.1. Age effects on new item recognition __ 104

IV.5.2. Age effects on old item recognition 106

IV.5.3. No age effects on the recognition performance as a function of memory strength 109 
V. Chapter: The antimuscarinic Agent, Biperiden selectively impairs Memory of the Abstract Figures without affecting the Processing of Non-Words

V.1. Abstract 112

V.2. Introduction 114

V.3. Methods 117

V.3.1. Participants

V.3.2. Study Design and Medication

V.3.3. Procedure 118

V.3.4. Data Analysis

V.4. Results 124

V.4.1. Abstract Figures 125

V.4.2. Non-Words 128

V.4.3. Complaints and POMS 130

V.5. Discussion and Conclusions 131

VI. Chapter: General Discussion 135

VI.1. Overview of the main Findings 137

VI.2. The current Paradigm in light of well-known Memory Phenomena and Aging 138

VI.3. The Impact of Memory Strength and Age on the Recognition Performance 141

VI.4. The pharmacological Model of healthy Aging 146

VI.5. Implications and Future Directions 149

VI.6. References 153

VI.7. Summary 169

VI.8. Impact Paragraph 173

VI.9. Acknowledgements 177

VI.10. Curriculum Vitae 178 
I. Chapter:

General Introduction and Outline
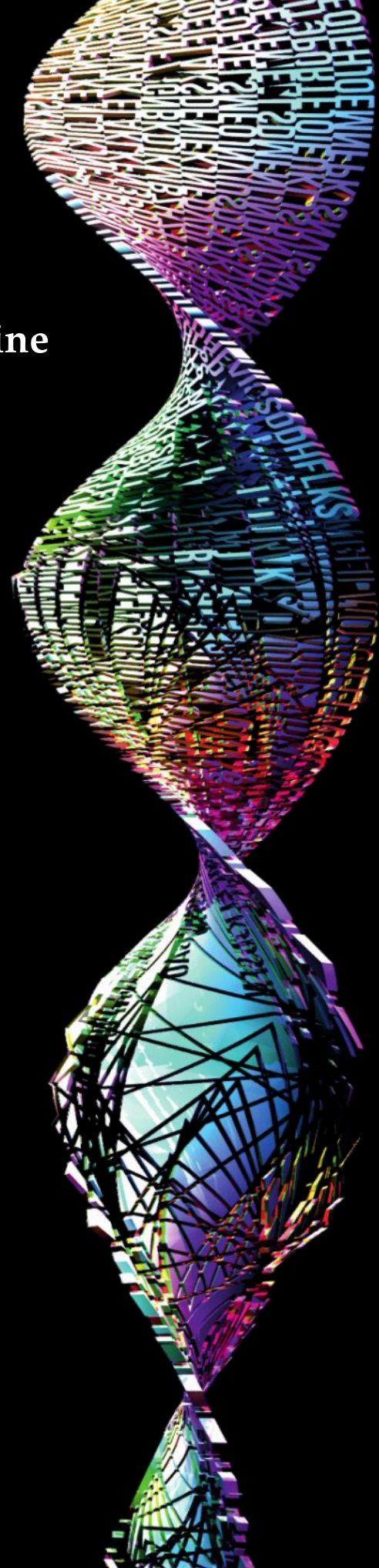

- Par

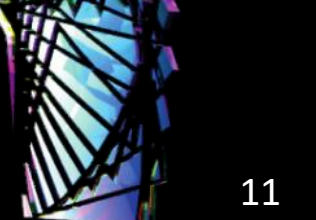




\section{I.1. Background}

Our brains are naturally geared towards novelty. Already as early as in infancy, newborns automatically react to novelty by showing an orienting response (Reynolds, Guy, \& Zhang, 2011), which signifies the importance of novelty processing in the brain. Furthermore, new stimuli and experiences divert attentional resources (Ranganath \& Rainer, 2003) and stimulate the brain's memory centers closely related to reward processing (Wittmann, Bunzeck, Dolan, \& Duzel, 2007). This is why experiencing something new may be more exciting and memorable than regularities. Such observations have been reinforced experimentally by evidence showing that memory can be better for new than previously encountered items (Beisteiner et al., 1996; Habib, McIntosh, Wheeler, \& Tulving, 2003; Tulving \& Kroll, 1995).

In support of this notion, electrophysiological and neuroimaging studies show an increased brain response to novelty. For example, increased brain activity has been reported in response to new compared to familiar stimuli reflected by the greater amplitude of the novelty-related components of the event-related potential (ERP) such as the frontal P300 and FN400 (Daffner et al., 2000; Daffner et al., 2006; Daffner et al., 2003). Also, neuroimaging studies found increased activation in brain areas crucial for stimulus evaluation and memory, the prefrontal cortex (PFC) and the structures of the medial temporal lobe (MTL), during the processing of novel relative to familiar items (Tulving, Markowitsch, Craik, Habib, \& Houle, 1996; Tulving, Markowitsch, Shitij, Habib, \& Sylvain, 1994). It has been suggested that increased processing efforts indicate that new information, relative to familiar information, is preferentially processed during encoding (novelty encoding hypothesis, NEH) (Kormi-Nouri, Nilsson, \& Ohta, 2005; Tulving, Markowitsch, Craik, Habib, \& Houle, 1996).

However, the natural desire for novelty can be counteracted by the fact that our brains become strongly habituated to the well-established and 
convenient "good old" routines, making one less likely to deviate from familiar experiences. The mnemonic advantages of familiarity relative to novelty have also been demonstrated in studies of recognition memory (Dobbins, Kroll, Yonelinas, \& Liu, 1998; Hintzman \& Curran, 1997; Poppenk, Kohler, \& Moscovitch, 2010; Ranganath \& Rainer, 2003). For example, the PFC and MTL showed increased activation in response to familiar items (Horn et al., 2016; Ramon \& Gobbini, 2017). Thus, the NEH is not easily reconciled with established findings showing mnemonic benefits of familiarity, such as more elaborate processing (Craik, 2002; Craik \& Lockhart, 1972; Craik \& Tulving, 1975) or repetition (Hintzman \& Curran, 1997). The proposal on the assumed advantages of novelty over familiarity is also complicated by evidence that the PFC and the MTL structure, the hippocampus, is sensitive to both novelty and familiarity (Bowman \& Dennis, 2015; de Chastelaine, Mattson, Wang, Donley, \& Rugg, 2017; Grady, McIntosh, \& Craik, 2003; Nyberg, 2005; Ranganath \& Rainer, 2003). Despite decades of research, there is still considerable controversy about which type of information, new or familiar, provides processing benefits.

Considering the involvement of the PFC and the MTL in information processing, it is not surprising that both healthy and pathological aging can impair the processing of new and familiar information. It is well-established that novelty detection (Fandakova, Lindenberger, \& Shing, 2014) and memory decline with age (Fraundorf, Hourihan, Peters, \& Benjamin, 2019) and are specifically impaired in conditions such as dementia and Alzheimer's disease (AD) (Ally et al., 2008). The most prominent reasons are brain atrophy and loss of neural connectivity in the PFC and the MTL (Grady, McIntosh, \& Craik, 2003; van Hooren et al., 2007). Although age-related memory deficits in tasks requiring novelty detection, recall, and context memory are well-researched (Fraundorf, Hourihan, Peters, \& Benjamin, 2019), differences in the processes underlying recognition memory and old/new assessments remain unclear. Therefore, the present work intended to further contribute to a better understanding of the 
factors involved in processing old and new information, including the perspective of aging.

\section{I.1.1. Old/new Recognition}

At first, it is essential to review and understand how the brain processes and responds to old and new stimuli. To identify something as new, the brain must distinguish between what is known (old) and what is not known (new). In this context, discrimination performance is typically investigated with recognition memory paradigms in which participants are required to recognize previously studied stimuli as 'old' correctly and identify not presented ones as 'new' (Malmberg, 2008). The recognition/discrimination process generates a cue that induces a sense of familiarity or novelty (Yonelinas, 2002; Yonelinas, Kroll, Dobbins, Lazzara, \& Knight, 1998). Behavioral data indicate that old/new discrimination at least partly depends on assessing the strength of a particular memory. Memory strength is assumed to vary on a continuum ranging from weak to strong, which underlies the subjective perception of stimulus familiarity (Wixted \& Stretch, 2004; Yonelinas, 2002). In other words, the stronger the stimulus memory is, the more robust the sense of familiarity appears, which points towards choosing an 'old' over a 'new' response.

Previous research has shown that memory strength can effectively be manipulated as a function of repetition (Verde \& Rotello, 2007) or the Levels of Processing (LOP) (Gardiner, 1988). Repetition contributes to the ease of access to a particular memory by increasing the subjective sense of familiarity resulting from repeated encoding (Hintzman \& Curran, 1997; Ranganath \& Rainer, 2003). The LOP theory predicts that deep (e.g., meaning-extraction, pattern recognition, activation of prior knowledge) and intermediate processing (e.g., phonetics) lead to superior and faster retrieval when compared to shallow processing (e.g., perceptual analyses, rehearsal) in young participants (Craik, 2002; Craik \& 
Lockhart, 1972; Craik \& Tulving, 1975; Newell \& Andrews, 2004). The processing benefits of repetition and LOP are also evident in the elderly (Park \& Festini, 2017) and in demented individuals (Ally, 2012).

The LOP framework attempted to explain the mnemonic system. Mnemonics, such as verbal (e.g., rhyming), motor (e.g., drawing), or visual (e.g., imagining) techniques, strengthen memory storage and improve subsequent retrieval as a result of deeper and more complex processing (Hulstijn, 1997; JonesGotman, 1986; Paivio \& Desrochers, 1981; Solso, 1995). Although the LOP theory has been criticized, it has changed memory research's direction by showing that encoding and long-term memory (LTM) storage are not simple but rather complex processes. Briefly, the criticism concerned the lack of definition of depth of processing and circularity (deep encoding improved memory performance, but memory itself was the outcome measure) (Ekuni, Vaz, \& Bueno, 2011; Eysenck, 1978a; Eysenck, 1978b). However, the advocates of the LOP argued that the complexity or robustness of encoding assures the creation and activation of multiple cues leading to the same memory trace during encoding and retrieval, respectively, which leads to better performance if stimuli are encoded more deeply (Craik, 2002; Craik \& Lockhart, 1972; Craik \& Tulving, 1975; Lockhart \& Craik, 1978). Nyberg (2002) reviewed neuroimaging studies to evaluate the LOP framework. The presented evidence indicated that certain brain regions that were active during encoding were indeed reactivated during retrieval. Thus, as Craik and Lockhart (1972) suggested, the complexity of encoding defined the memory trace. Consequentially, the LOP provides evidence that the intention to learn is less critical than encoding quality for successful retrieval. Such findings delineate the role of memory strength as a mediator of successful old/new discrimination. 


\section{I.1.2. The Impact of the Stimulus on old/new Recognition}

Over the past, memory researchers have primarily focused on understanding the processing of pre-experimentally well-known stimuli, such as meaningful pictures and words. Such items involve semantics and likely rely on pre-experimentally existing memories, which entails a lifespan of experiences, especially in older people. As such, pre-experimentally familiar stimuli presumably activate existing memory traces, re-encoded in the experimental context, forming slightly different engrams (Poppenk, McIntosh, Craik, \& Moscovitch, 2010). The pre-existing and newly created memory traces can interfere (Poll et al., 2020).

In this context, past experiences or pre-experimental familiarity can facilitate or hinder discrimination performance (Umanath \& Marsh, 2014). The propositional network account can explain the former. According to this theory, prior knowledge may facilitate higher-order processes in LTM (Anderson, 1983). The latter can be the result of either pro- or retroactive interference (Izawa, 1980). As such, when pre-experimental experience exists, separation of internal and external experimental information sources can become difficult. In other words, even in a simple old/new recognition test, source memory for familiar items can lead to confusion as to whether one saw the item in or outside of an experimental setting. Indeed, source confusion has previously been identified as a confounder in tasks involving recognition (Dobbins, Kroll, Yonelinas, \& Liu, 1998). Although aged adults tend to use their existing semantic knowledge more efficiently than the young, they can be even more prone to source confusion reflected by the elderly typically being more biased towards judging an item 'old' even when it is new (Bowman \& Dennis, 2015; Fraundorf, Hourihan, Peters, \& Benjamin, 2019).

In contrast to meaningful, pre-experimentally familiar items, the processing of pre-experimentally unfamiliar stimuli, such as abstract figures and non-words, is poorly understood. Such stimuli are characterized as pre- 
experimentally not seen combinations of simple shapes (abstract figures) and letters (non-words) without a meaning. However, knowing how the brain processes and discriminates pre-experimentally unfamiliar and meaningless stimuli can significantly improve our understanding of visual, lexical, and orthographic memory processing and contribute to designing relevant aging models. Furthermore, the application of such stimuli reduces the potential confounding effects of retroactive interference, and source confusion as memories must be newly formed (Norman, Tepe, Nyhus, \& Curran, 2008). Thus, pure learning and memory effects can be isolated. For example, Koutstaal, Reddy, Jackson, Prince, Cendan, and Schachter (2003) used a recognition memory paradigm with meaningless abstract pictures that were either labeled with familiar words (e.g., bread) or not. They found that false alarms increased, and accuracy decreased when meaningful labels were used compared to when no labels were applied.

Contrasting findings were reported by Belleville, Menard, and Lepage (2011). They tested the discrimination performance of well-known vs. novel verbal (e.g., words and pseudo-words) and auditory stimuli (e.g., well-known and novel melodies) with a two-phase study-recognition memory paradigm in a population of young, healthy elderly, and demented patients with mild cognitive impairment $(\mathrm{MCI})$. The healthy young group's results indicated that in both the verbal and the musical domains, recognition and recollection ratings were superior for the well-known compared to the novel items, which the authors attributed to the well-known stimuli being agreeable to the type of elaborative encoding required for recollection. Their results also showed that healthy aging impaired the recognition of well-known items, whereas MCI impaired novelty detection. Also, healthy older adults compared to the young were impaired in recollection and familiarity, while MCI patients were even more impaired in recollection than healthy older adults. The authors argued that the recollection deficit in healthy aging and MCI aligns with elaborate encoding difficulties. Thus, 
these findings further delineate the differential impact of encoding and preexperimental familiarity on the discrimination process.

\section{I.1.3. Modelling Age-related Memory Impairments with Biperiden}

Previous research has shown that the neurotransmitter acetylcholine (ACh) is essential for memory (Hasselmo, 2006; Hasselmo, Wyble, \& Wallenstein, 1996) and novelty detection (Caldenhove, Borghans, Blokland, \& Sambeth, 2017; Rangel-Gomez \& Meeter, 2016). It is also known that aging and dementia negatively affect old/new recognition performance (Fraundorf, Hourihan, Peters, \& Benjamin, 2019), likely resulting from a combination of poor new (Bastin, Delhaye, Moulin, \& Barbeau, 2019) and old item recognition, especially when recollection type memory is involved (Dalla Barba, 1997). Such processing problems necessitate the establishment of episodic memory impairment models that can aid the development of cognitive enhancers.

Recent studies have proposed a relatively specific cholinergic memory deficit model induced by biperiden (BIP), a medication used as an adjunct treatment in Parkinson's disease (Blokland, Sambeth, Prickaerts, \& Riedel, 2016; Kostelnik, Cegan, \& Pohanka, 2017). Both 2 and 4 mg BIP have previously been shown to induce dementia-like episodic memory impairment in healthy volunteers (Borghans, Sambeth, \& Blokland, 2020; Borghans, Blokland, \& Sambeth, 2017; Borghans, Sambeth, Prickaerts, Ramaekers, \& Blokland, 2018; Sambeth, Riedel, Klinkenberg, Kahkonen, \& Blokland, 2015; Wezenberg, Verkes, Sabbe, Ruigt, \& Hulstijn, 2005). Such findings may suggest that BIP could induce a memory impairment, as seen in aging. Whether the effects of BIP also mimic the age-related deficits on recognition memory has not been investigated yet. 


\section{I.2. The Aim and Approach of the current Dissertation}

This dissertation aimed to investigate further the processes underlying old/new recognition, focusing on the processing of pre-experimentally unfamiliar abstract figures and non-words. It was of specific interest to examine the role of memory strength and age in such processing. However, there are hardly any studies investigating the old/new discrimination processing using abstract figures and non-words especially considering the impact of memory strength. Thus, the forthcoming studies are to a great extent exploratory. In this dissertation, a three-phase old/new recognition memory paradigm was used to characterize the recognition performance of pre-experimentally unfamiliar items in healthy young volunteers. Next, the underlying neural correlates are investigated using EEG methods. Subsequently, the same paradigm was tested in healthy elderly participants to reveal age-related processing effects. Lastly, it was tested whether the selective muscarinic antagonist BIP could model the ageassociated deficit in healthy aging.

\section{I.3. Outline of the Dissertation}

In Chapter II, old/new recognition is characterized in healthy young volunteers. This behavioral study investigated the recognition performance of pre-experimentally familiar (figures and words) and unfamiliar (abstract figures and non-words) items in a sample of 31 healthy young adults. Manipulations involving the LOP and repetition were used to examine the possible role of preexperimental familiarity and memory strength in the recognition process. Additionally, a control experiment was conducted to detect the effects of different item proportions (equal and unequal) on the response bias. Here, 43 participants were tested. In Chapter III, electroencephalography (EEG) was used to understand which physiological processes can explain the behavioral findings. Novelty components and early and late old/new effects were examined for the 
pre-experimentally unfamiliar abstract figures and non-words during recognition in a sample comprising 22 healthy young adults. In Chapter IV, it was examined how aging affects recognition memory using the previously characterized paradigm. In this experiment, the performance of 15 healthy young and 16 healthy elderly was compared to reveal any age effects. The final study, Chapter $V$, tested in 20 volunteers whether $4 \mathrm{mg}$ BIP could be a suitable model to induce memory impairment using a behavioral approach. Lastly, in Chapter VI, an overview of the main findings of this dissertation is given with implications for future research. Of note: there is a partial overlap concerning some of the volunteers assessed in the different experiments described in this dissertation. An overview of the recruited and tested participants without exclusions is given in Table 1.

Table 1: Sample overview for each Chapter/Experiment

\begin{tabular}{|l|l|l|}
\hline Chapter & \multicolumn{2}{|c|}{ Participants } \\
\hline II & Pre-experimentally familiar, $(n=18)$ & $\begin{array}{l}\text { Pre-experimentally unfamiliar, }(n= \\
15)\end{array}$ \\
\hline II (appendix) & Equal proportions, $(n=22)$ & $\begin{array}{l}\text { Unequal proportions, }(n=22, \text { out of } \\
\text { which } 15 \text { the same as in the pre- } \\
\text { experimentally unfamiliar group in } \\
\text { Chapter II })\end{array}$ \\
\hline III & $\begin{array}{l}\text { Healthy young, }(n=22, \text { same as the unequal proportions group in the appendix } \\
\text { of Chapter II })\end{array}$ \\
\hline IV & $\begin{array}{l}\text { Healthy young, }(n=15, \text { same as the } \\
\text { pre-experimentally unfamiliar group in } \\
\text { Chapter II })\end{array}$ & Healthy elderly, $(n=15)$ \\
\hline V & \begin{tabular}{l} 
Healthy young, $(n=21)$ \\
\hline
\end{tabular}
\end{tabular}

$n$ : recruited and tested sample size without exclusions 


\section{Chapter:}

The Role of pre-experimental

Familiarity and Memory Strength in old/new Recognition

Monika Toth, Anke Sambeth \& Arjan Blokland

To be submitted for publication

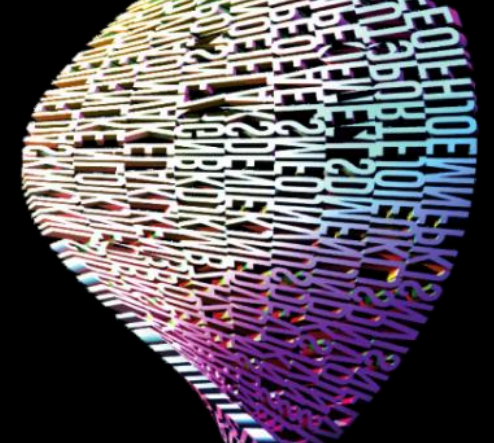




\section{II.1. Abstract}

The mnemonic benefits of stimulus novelty and familiarity in recognition memory tasks are part of considerable debate. Here, we considered the role of memory strength and pre-experimental familiarity using a three-phase old/new recognition memory paradigm (deep memorization, shallow memorization, and recognition). Memory strength was manipulated as a function of the Levels of Processing. Strong memories were induced with deep encoding and repetition, while weak memories were achieved via shallow encoding without repetition. To account for the effects of pre-experimental familiarity, we used preexperimentally familiar and unfamiliar visual and verbal materials (figures and words vs. abstract figures and non-words). We found that recognition performance primarily depends on experimentally induced memory strength. No differences were found between pre-experimental familiar and unfamiliar old item recognition accuracy. However, the new, unfamiliar figures were recognized better than the familiar ones, probably due to the more profound distinctiveness of the former. Additionally, the response times were also faster to the familiar than the abstract figures. Importantly, we found that new item identification exceeded the correct old item recognition when the memory was weakly embedded but not when it was strongly embedded. No differences were found between the pre-experimentally familiar and unfamiliar stimuli. Thus, this study indicates that while pre-experimental familiarity has a differential impact on visual and verbal materials. This is not the case when it comes to memory strength.

Keywords: Levels of Processing; memory strength; recognition memory; preexperimental familiarity; abstract figures; non-words; figures; words 


\section{II.2. Introduction}

A memory system is efficient if it can detect and integrate new information and readily retrieve familiar information (Donaldson, 1992; Kafkas \& Montaldi, 2014; Kafkas \& Montaldi, 2018; Kumaran \& Maguire, 2007). In this context, information-processing theories define recognition as the ability to correctly discriminate between familiar and new materials (Benjamin, Diaz, \& Wee, 2009; Stanislaw \& Todorow, 1999; Verde \& Rotello, 2007; Yonelinas, 2002). Discrimination performance is typically investigated with paradigms that assess recognition memory. In these paradigms, participants are asked to judge a probe item as 'old' if they think they saw it before or as 'new' if they consider the item new to them (Malmberg, 2008). Thus, the probe item generates a cue during decision making, which initiates a sense of familiarity in case there is a match to an existing representation or novelty if there is no match (Yonelinas, 2002; Yonelinas, Kroll, Dobbins, Lazzara, \& Knight, 1998). Thus, novelty/familiarity responses rely on the judgment concerning previous encounters.

Psychological theories of recognition memory have been the subject of considerable debate. For example, some scientists argued that familiarity provides a mnemonic advantage (Poppenk, Kohler, \& Moscovitch, 2010), whereas others advocated the benefits of novelty (Beisteiner et al., 1996; Habib, McIntosh, Wheeler, \& Tulving, 2003). For instance, Poppenk, Kohler, and Moscovitch (2010) used a three-phase old/new recognition paradigm with proverbs. They found that the recognition of the familiarized compared to the new items was superior. In contrast, Beisteiner et al. (1996) used abstract figures and non-words in a continuous recognition paradigm. They found that new items were recognized more accurately than the old ones. Irrespective of these inconsistent findings on recognition, there is a consensus that responses to new compared to old stimuli usually take longer (Delhaye, Bastin, Moulin, Besson, \& Barbeau, 2017; Kafkas \& Montaldi, 2014; Kafkas \& Montaldi, 2018). The present 
study intended to further contribute to a better understanding of the recognition processes, especially concerning the materials used. For this reason, we considered the impact of memory strength and pre-experimental stimulus familiarity.

First, the memory strength framework suggests that, in discrimination tasks, the probe items are compared to the existing memory representations, resulting in a strong, weak, or no match. Memory strength is defined as the ease of access to a particular memory (Benjamin, Diaz, \& Wee, 2009; Hirshman, 1995; Stanislaw \& Todorow, 1999; Verde \& Rotello, 2007; Yonelinas, 2002). Previous studies have shown that memory strength can effectively be manipulated as a function of repetition (Verde \& Rotello, 2007) or the Levels of Processing (LOP) (Gardiner, 1988). Repetition is known to strengthen memory by increasing the subjective sense of familiarity resulting from re-encoding of a particular memory trace (Hintzman \& Curran, 1997; Ranganath \& Rainer, 2003). The LOP theory predicts that deep (e.g., meaning-extraction, pattern recognition, activation of prior knowledge) and intermediate processing (e.g., phonetics) lead to superior and faster retrieval when compared to shallow processing (e.g., perceptual analyses, rehearsal) (Craik, 2002; Craik \& Lockhart, 1972; Craik \& Tulving, 1975; Newell \& Andrews, 2004). Moreover, previous research has shown that mnemonics, such as verbal (e.g., rhyming), motor (e.g., drawing), or visual (e.g., imagining) techniques, strengthen memory storage and improve subsequent retrieval as a result of deeper processing (Hulstijn, 1997; Jones-Gotman, 1986; Paivio \& Desrochers, 1981; Solso, 1995).

Second, past experiences or pre-experimental familiarity can aid or hinder discrimination performance (Umanath \& Marsh, 2014). The former can be explained by the propositional network account according to which prior knowledge may facilitate higher order processes in utilizing long-term memory (LTM) (Anderson, 1983). The latter can be the result of either pro- or retroactive interference (Izawa, 1980). Thus, when pre-experimental experience exists, 
separation of internal and external experimental information sources can become difficult. In other words, even in a simple old/new recognition test, source memory for familiar items can lead to confusion as to whether one saw the item in or outside of an experimental setting. The above can lead to errors such as false alarms (i.e., incorrect identification of new items as 'old'), which decreases the recognition performance (Malmberg, 2008). For example, Belleville, Menard, and Lepage (2011) tested the discrimination performance of well-known vs. novel verbal (e.g., words and pseudo-words) and auditory stimuli (e.g., well-known and novel melodies) with a two-phase study-recognition memory paradigm. The results of the healthy young group indicated that in both the verbal and the musical domains, recognition was superior for the well-known compared to the novel items, which the authors attributed to the well-known stimuli being agreeable to the type of elaborative encoding required for recollection.

Pre-experimental stimulus familiarity can strongly influence memory performance and be problematic when stimuli rely on different degrees of memory strength. For example, high-frequency words, which imply higher preexperimental familiarity due to frequent use in everyday language, were shown to be encoded better than low-frequency words (Kim \& Glanzer, 1993; Reder et al., 2000). Not only the frequency of use but associations and linked experiences to pre-experimentally familiar items cam facilitate memory. For example, the word "holiday" is typically associated with positive emotions. These associations might strengthen the encoding of the word "holiday", while a more neutral word, such as "rock", is not associated with pleasant pre-experimental experiences and is likely to be encoded weaker. When using pre-experimentally familiar words, pictures or faces, pre-experimental familiarity increases the memory performance for those stimuli (Gardiner \& Java, 1990; Yonelinas, 2002).

To evaluate the possible role of these factors, we used a three-phase old/new memory paradigm (Toth, Sambeth, \& Blokland, 2021). To examine whether pre-experimental familiarity affects recognition performance, we used 
pre-experimentally unfamiliar abstract figures and non-words, and preexperimentally familiar figures and words. The latter most probably had an already existing representation in memory, whereas the former likely did not. The use of pre-experimentally unfamiliar stimuli enabled controlling for potential confounding effects of retroactive interference as their memories had to be newly formed (Norman, Tepe, Nyhus, \& Curran, 2008). We chose to investigate visual and verbal stimuli since these are assumed to be processed differently (Kim, Yoon, \& Park, 2004), likely because their processing engages different brain regions (Khateb, Pegna, Michel, Landis, \& Annoni, 2002). Hence, by including both visual and verbal materials, we could test whether the effects of memory strength and pre-experimental familiarity are general or stimulusdependent.

Taking the above into consideration, we anticipated for both the preexperimentally unfamiliar and familiar groups that the deeply encoded and repeated items relying on strong memories would be recognized better and faster than the shallowly encoded ones having weak memory representations. However, since the current paradigm was exploratory and previous results are mixed, we did not have specific expectations regarding the differences in recognition of the pre-experimentally familiar and unfamiliar visual and verbal materials.

\section{II.3. Methods}

The Ethical Committee of the Faculty of Psychology and Neuroscience of Maastricht University granted ethical approval for this experiment. Each participant received monetary compensation or research participation credit points. Ethical Approval Codes: ECP13_02_2012 and ERCPN-211_01_08_2019. 


\section{II.3.1. Participants}

The main inclusion criteria were age (18-30 years) and being fluent in the Dutch and English languages. Participants were recruited by means of advertising. For the pre-experimentally familiar group, a total of 18 participants were recruited. However, two participants were excluded from further analyses due to technical issues during recording. The final sample contains data of 16 participants (9 males) with average age of 22 years. A total of 15 young healthy participants (5 males) with mean age of 23 years were recruited and analyzed for the pre-experimentally unfamiliar group.

\section{II.3.2. Procedure}

After signing informed consent, participants were admitted to the study. Prior to starting the experiment, each participant filled in a demographic questionnaire, including information about sex, age, and handedness. During the test, stimuli were presented via a computer screen, and participants had to respond on two keys of a response pad. Recognition accuracies and reaction times were recorded.

A memory paradigm with visual (abstract figures or figures) and verbal items (non-words or words) was applied in separate tests. See Figure 1 for an example of the stimuli used. Every participant performed each test phase first with the abstract figures and then with the non-words (pre-experimentally unfamiliar group) or the figures and then with the words (pre-experimentally familiar group) to minimize verbalization of the figurative stimuli. The experiment consisted of three phases (see Figure 2). In phase 1 (deep memorization leading to 'strong' memory), participants were familiarized with a series of 30 visual or monosyllabic verbal stimuli in separate tests (list 1: L1). Participants were asked to manually redraw the visual items on an answer sheet to induce deep LOP. They had to mention existing English or Dutch rhyming 
words for each non-word to induce intermediate LOP. Stimuli were presented for $1 \mathrm{~s}$, and the participants were given $14 \mathrm{~s}$ to execute the mnemonic encoding task. If they were ready earlier, they could press a button, and $2 \mathrm{~s}$ later, the next stimulus appeared. Stimuli were extracted from previous studies (Glosser, Friedman, Grugan, Lee, \& Grossman, 1998; Redoblado, Grayson, \& Miller, 2003; Seidenberg, Plaut, Petersen, McClelland, \& McRae, 1994).

\begin{tabular}{|c|c|c|c|c|c|}
\hline \multicolumn{3}{|c|}{ Familiar Figures } & \multicolumn{3}{|c|}{ Familiar Words } \\
\hline$\Leftrightarrow$ & 3 & $\mathscr{N}$ & LEAF & PRIDE & MEAL \\
\hline \multicolumn{3}{|c|}{ Abstract Figures } & \multicolumn{3}{|c|}{ Non-Words } \\
\hline r & $\tilde{0}$ & *ै & WAPS & KNANT & ZEIG \\
\hline
\end{tabular}

Fig. 1. Examples of the stimuli used. 


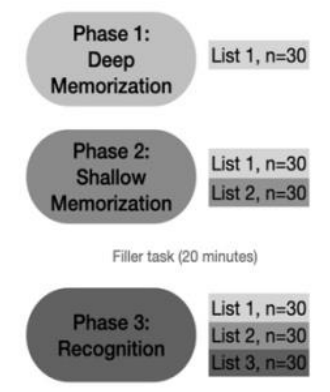

Fig. 2. Schematic overview of the experimental design. Phase 1: deep memorization with the preexperimentally unfamiliar abstract figures and non-words or with pre-experimentally familiar figures and words in separate tests using a mnemonic encoding task (redrawing the visual and mentioning rhyming words for the verbal stimuli). The 30 stimuli used here form List 1 (drawn/semantically processed stimuli). Phase 2: shallow memorization with the instruction to remember as many stimuli as possible. This phase contained items from List 1 and 30 new ones (List 2, studied stimuli). Phase 3: recognition of the stimuli including List 1, List 2, and 30 new (List 3). n: number of stimuli presented.

During phase 2 (shallow memorization leading to 'weak' memory), participants were instructed to remember as many stimuli as possible. In this phase, 60 stimuli (visual or verbal items) were used: 30 stimuli from L1 were randomly mixed with 30 new ones (L2). All stimuli were shown for $1 \mathrm{~s}$ with an inter-stimulus interval (ISI) of $2 \mathrm{~s}$.

During phase 3, participants were asked to decide if they had seen the presented stimulus in the previous series (L1 and L2) or whether the stimulus was new to them (L3: new, $n=30$ ). The 90 visual or verbal stimuli were presented for a duration of $1 \mathrm{~s}$, or less in the case of faster button press; the ISI was $2.5 \mathrm{~s}$. Participants had to press the corresponding buttons ('old' for L1 and L2, or 'new' for L3 stimuli) on a response box as quickly and accurately as possible. 
A filler paper-and-pencil task of $10 \mathrm{~min}$ and another non-verbal task were given between phase 2 and 3 . The filler task consisted of the localization of number sequences, vertically or horizontally placed within a field of numbers (10 min). The other task consisted of watching a silent cartoon while auditory stimuli were presented $(10 \mathrm{~min})$.

\section{II.3.3. Data Analysis}

Before analysis, all data were evaluated for having normal distribution and homogeneity of variance. Additionally, raw data were checked for outliers. Outlier values were replaced with their regression estimates produced by the missing value analyses (IBM SPSS Statistics for Macintosh, Version 27.0. Armonk, NY: IBM Corporation). Additionally, due to technical issues, 1-2 responses per participant were missing (e.g., the button press was not recorded). In these cases, values were replaced with their regression estimates. Effect sizes are reported based on partial eta-squared ( $\eta p 2)$ data. Furthermore, Mauchly's test of sphericity was applied. In case the assumption of sphericity was violated, a GreenhouseGeisser correction was used. In all cases, degrees of freedom of assumed sphericity were reported. Levene's test was used to assess the equality of variances. In case the assumption of equal variances was violated, median-based independent samples non-parametric tests were applied. Post-hoc comparisons and simple effects were investigated using paired-samples and independents samples t-tests, applying adjustments for multiple comparisons; the observed pvalues were multiplied by the number of comparisons, which was tested against the set significance level of 0.05 . Values of unequal variances are reported if the assumption of equal variances was violated.

For the behavioral data, Signal Detection Theory (SDT) was applied to investigate the discrimination performance (Benjamin \& Bawa, 2004; Benjamin, Diaz, \& Wee, 2009; Stanislaw \& Todorow, 1999; Verde \& Rotello, 2007). 
Discrimination accuracy was defined as the ability to distinguish the different types of stimuli (drawn/semantically processed, studied and new). Recognition accuracy was defined as the ability to distinguish the different types of stimuli (drawn/semantically processed, studied, and new). Correct responses included an 'old' response to the drawn/semantically processed items, and the studied stimuli, and a 'new' response to the new items. Incorrect responses involved a 'new' response to the drawn/semantically processed items and the studied stimuli and an 'old' response to the new stimuli. See Table 1 for an overview.

Table 1. Overview of the different types of responses as a function of stimulus type.

\begin{tabular}{ccc}
\hline & Stimulus Type & Response \\
\hline Hit (H) & $\begin{array}{c}\text { Drawn or semantically } \\
\text { processed/Studied }\end{array}$ & 'Old' \\
\hline Miss (M) & $\begin{array}{c}\text { Drawn or semantically } \\
\text { processed/Studied }\end{array}$ & 'New' \\
\hline Correct Rejection (CR) & New & 'Old' \\
\hline False Alarm (FA) & New & H/(H + M) \\
\hline Hit Rate (HR) & Drawn or semantically \\
\hline processed/Studied & New & $\mathrm{CR} /(\mathrm{CR}+\mathrm{FA})$ \\
\hline
\end{tabular}

Given the memory strength manipulation in the current design (deep memorization, shallow memorization, and recognition), the correct response rates, being hit rates (HR) for the drawn/semantically processed, and the studied items and correct rejection rates (CRR) for the new, were used to evaluate the discrimination accuracy. Furthermore, in order to investigate discriminability, non-parametric $\mathrm{A}^{\prime}$ statistics were computed for the drawn/semantically processed and the studied stimuli using Equations (1) or (2) (see below). A' varies from 0 to 1 , with 0.5 indicating chance performance. Higher values are indicative of improved performance (Snodgrass \& Corwin, 1988; Stanislaw \& Todorow, 1999). 


$$
\begin{aligned}
& \mathrm{A}^{\prime}=0.5+\frac{(H R-F A R)(1+H R-F A R)}{4 H R(1-F A R)}, \text { if } H R \geq F A R \\
& \mathrm{~A}^{\prime}=0.5-\frac{(H R-F A R)(1+H R-F A R)}{4 H R(1-F A R)}, \text { if } H R<F A R
\end{aligned}
$$

A': discriminability index, HR: hit rate, FAR: false alarm rate.

During recognition, the a priori probabilities of old and new items and the quality of the match between a test item and the memory for studied items can influence the bias parameter (Huang \& Ferreira, 2020; Stanislaw \& Todorow, 1999). Such a model does not fit the current paradigm due to the memory strength manipulation used and the equivalent proportion and intended comparison of the strong $(n=30)$, weak $(n=30)$, and new items $(n=30)$ [44]. After all, the final proportion of 'old' and 'new' responses was 2:1. Therefore, we calculated the total amount of 'old' $(\mathrm{H}+\mathrm{FA})$ and 'new' $(\mathrm{M}+\mathrm{CR})$ responses given by the participants. This was done to examine whether there was a preference for either the 'old' or 'new' responses. Results were compared using paired samples t-tests with Bonferroni corrections.

RT data of the hits were evaluated, as well. To be able to use parametric tests, RT-s were transformed into $|\log (1 / \mathrm{RT})|$ to obtain a normal distribution of the data (Osborne, 2002). Moreover, the median RT data are reported as central tendency parameters, together with the corresponding first and third interquartile ranges (Ratcliff, 1993).

Statistical analysis was conducted using SPSS 27.0. A repeated measures analysis of variance (ANOVA) was used to investigate discrimination accuracy scores and RT-s for the different stimuli in the different categories as assessed in Phase 3. The within-subject variables were stimulus type (drawn/semantically processed, studied stimuli, and new) for the abstract figures and non-words analyzed in separate tests, and the between subject variable was preexperimental familiarity (pre-experimentally familiar and pre-experimentally unfamiliar). 


\section{II.4. Results}

Although there was an unequal number of old-responses over newresponses (2:1), we found that there was no response bias except for the preexperimentally familiar old figures (see Table 2). However, it is essential to mention that $2 \%$ of the old responses in this experiment were missing because no button press was recorded. The mean signal-detection parameter estimates are displayed in Table 3.

Table 2. The total number of old and new responses during the recognition test. Data represent the means (SEM) of the total 'old' and 'new' responses and the corresponding \% compared to the 90 items/stimulus category (visual and verbal), and the t-statistics for the pre-experimentally familiar and pre-experimentally unfamiliar groups.

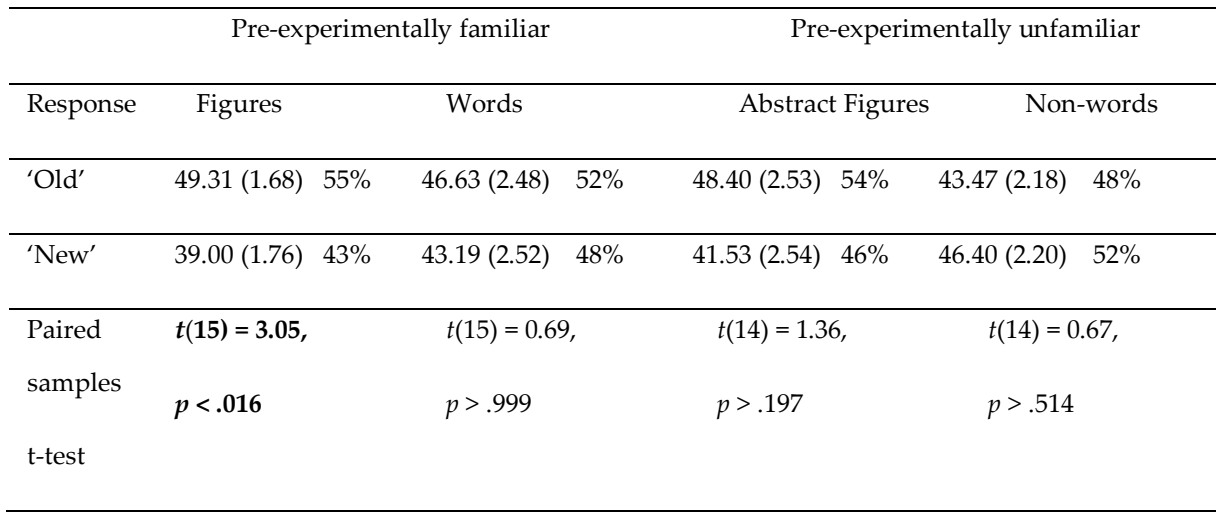


Table 3. Means (SEMs) of the signal-detection measures of the recognition performance of the visual and verbal materials according to stimulus type (drawn/semantically processed, studied, and new) for the pre-experimentally familiar and pre-experimentally unfamiliar groups.

\begin{tabular}{|c|c|c|c|c|c|}
\hline \multirow[b]{2}{*}{ Stimulus } & \multirow[b]{2}{*}{ Parameters } & \multicolumn{2}{|c|}{ Pre-experimentally familiar } & \multicolumn{2}{|c|}{ Pre-experimentally unfamiliar } \\
\hline & & Figures & Words & Abstract Figures & Non-words \\
\hline \multicolumn{6}{|l|}{ Type } \\
\hline \multirow{2}{*}{$\begin{array}{l}\text { Drawn/Se } \\
\text { mantically } \\
\text { processed }\end{array}$} & HR & $0.96(0.01)^{a a, b b}$ & $0.77(0.05)^{a a}$ & $0.98(0.01)^{a a, b b}$ & $0.80(0.04)^{a a}$ \\
\hline & $\mathrm{A}^{\prime}$ & $0.88(0.01)^{a a}$ & $0.67(0.02)^{a a}$ & $0.90(0.10)^{a a}$ & $0.69(0.02)^{a a}$ \\
\hline \multirow[t]{2}{*}{ Studied } & HR & $0.60(0.05)$ & $0.58(0.04)$ & $0.57(0.06)$ & $0.50(0.04)^{b b}$ \\
\hline & $\mathrm{A}^{\prime}$ & $0.62(0.02)$ & $0.55(0.01)$ & $0.62(0.02)$ & $0.55(0.01)$ \\
\hline New & CRR & $0.89(0.02) a a$ & $0.85(0.02)$ & $0.95(0.01) * a a$ & $0.84(0.02)$ \\
\hline
\end{tabular}

HR: hit rate, CRR: correct rejection rate, $\mathrm{A}^{\prime}$ : discriminability index. Pre-experimental familiarity effects: ${ }^{*}: p<.05$. Stimulus type effects: different from the studied items: aa $p<.001$, different from the new items: bb $p<.001$.

\section{II.4.1. Visual Stimuli}

When analyzing the accuracy performance with the figures and the abstract figures the ANOVA revealed a main effect of stimulus type $[F(2,58)=$ 90.59, $\eta p^{2}=0.76, p<.001$; see Fig. $3 \mathrm{~A}$ and Table 3]. Post-hoc tests showed the drawn items were recognized more accurately than the studied $(p<.001)$ and new $(p<.001)$. Also, more new stimuli were endorsed correctly compared to the studied $(p<.001)$. Levene's test indicated unequal variances for the new abstract figures $[F(1,29)=7.91, p<.009]$. The non-parametric test revealed that the preexperimentally unfamiliar new abstract figures $(M d n=0.93)$ were recognized better than the pre-experimentally familiar new ones $(M d n=0.90)[U=192.50, p$ $<.004$; see Fig. 3A]. This was not found with the drawn $[U=149.00, p>.222]$ or studied visual items $[U=110.00, p>.692]$. Finally, the interaction term pre- 
experimental familiarity $\mathrm{x}$ stimulus type was not significant $\left[F(2,28)=1.64, \eta p^{2}=\right.$ $0.11, p>.212]$.

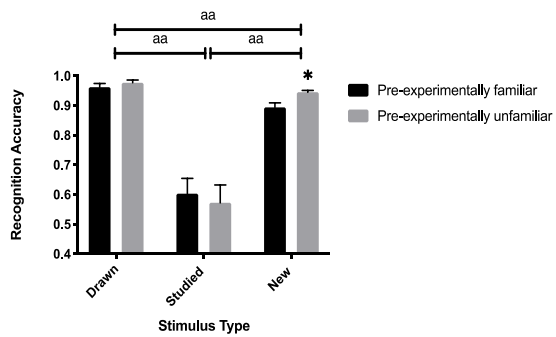

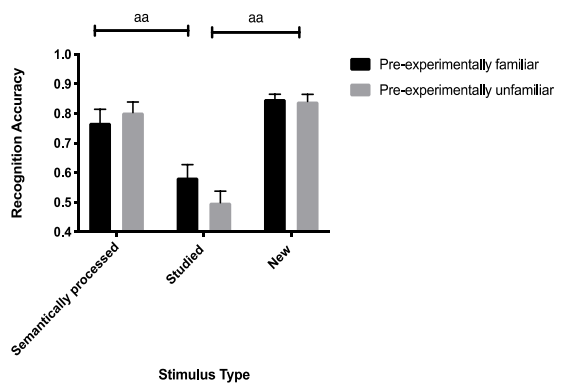

Fig 3. Recognition accuracy performance of the visual (A) and the verbal items (B) according to stimulus type and pre-experimental familiarity. The bars represent the means of the hit rates of the drawn/semantically processed and the studied items, and the means of the correct rejection rates for the new items. Pre-experimental familiarity effects: ${ }^{*}: p<.05$. Stimulus type effects: aa: $p<.001$.

The analyses performed on the $\mathrm{A}^{\prime}$ scores revealed that the drawn figures were discriminated significantly better than the studied $\left[F(1.29)=324.61, \eta p^{2}=\right.$ 0.92, $p<.001$; see Table 3]. The pre-experimental familiarity $\mathrm{x}$ stimulus type interaction was not significant $\left[F(1,29)=3.10, \eta p^{2}=0.10, p>.089\right]$, and there was no effect of pre-experimental familiarity $\left[F(1,29)=1.51, \eta p^{2}=0.05, p>.229\right]$.

When analyzing the reaction time performance a main effect of preexperimental familiarity was revealed indicating that overall reactions were faster to the pre-experimentally familiar compared to the unfamiliar visual stimuli $\left[F(1,29)=6.23, \eta p^{2}=0.18, p<.018\right.$; see Table 4]. Another main effect of stimulus type was found $\left[F(2,28)=84.65, \eta p^{2}=0.86, p<.001\right.$; see Table 4]. Posthoc tests showed that response times were significantly faster to the drawn stimuli compared to the studied $(p<.001)$ and new items $(p<.001)$. No such difference was found between the studied and new stimuli $(p>$.999). Finally, the 
stimulus type $\mathrm{x}$ pre-experimental familiarity interaction was statistically not meaningful $\left[F(2,28)=2.00, \eta p^{2}=0.13 p>.154\right]$.

Table 4. Median reaction times (middle 50\% range; in milliseconds in response to the drawn/semantically processed, studied, and new abstract figures and non-words) and their corresponding first and third interquartile ranges for the pre-experimentally familiar and unfamiliar groups.

\begin{tabular}{lcccc}
\hline \multicolumn{2}{c}{ Pre-experimentally familiar } & \multicolumn{2}{c}{ Pre-experimentally unfamiliar } \\
\hline Stimulus Type & Figures & Words & Abstract Figures & Non-words \\
\hline Drawn/Semanti & $638^{*}$ & 621 & 661 & 648 \\
cally processed & $(568-706)$ & $(574-654)$ & $(661-742)$ & $(636-665)$ \\
\hline Studied & $722^{*}, a a$ & 609 & $794 a a$ & $(575-719)$ \\
& $(650-802)$ & $(573-650)$ & $(718-1011)$ & 689 \\
\hline New & $701^{*}, a a$ & 645 & $811^{a a}$ & $(637-734)$ \\
& $(676-805)$ & $(589-688)$ & $(734-953)$ & \\
\hline
\end{tabular}

Pre-experimental familiarity effects: ${ }^{*} p<.05$. Stimulus type effects: different from the drawn/semantically processed: aa $p<.001$, different from the studied: $b b p<.001$.

\section{II.4.2. Verbal Stimuli}

The ANOVA analysis for the verbal stimuli revealed a main effect of stimulus type $\left[F(2,28)=48.06, \eta p^{2}=0.77, p<.001\right.$; see Fig. 3B]. Post-hoc tests showed that overall, the semantically processed items were recognized better than the studied $(p<.001)$ but not compared to the new ones $(p>.283)$. Also, more new stimuli were identified correctly compared to the studied $(p<.001)$. Neither pre-experimental familiarity $\left[F(1,29)=0.35, \eta p^{2}=0.01, p>.558\right]$ nor the interaction term pre-experimental familiarity $\mathrm{x}$ stimulus type were found to be significant $\left[F(2,28)=2.10, \eta p^{2}=0.13, p>.144\right]$. 
The analyses performed on the $\mathrm{A}^{\prime}$ scores revealed that the semantically processed verbal items were discriminated significantly better than the studied $\left[F(1.29)=77.51, \eta p^{2}=0.73, p<.001\right.$; see Table 3]. The pre-experimental familiarity $\mathrm{x}$ stimulus type interaction was not significant $\left[F(1,29)=2.15, \eta p^{2}=0.07, p>.154\right]$, and there was no effect of pre-experimental familiarity $\left[F(1,29)=0.02, \eta p^{2}=0.01\right.$, $p>$.887]

In the session with the non-words the reaction times were similar for all stimulus types $\left[F(2,28)=3.46, \eta p^{2}=0.20 p>.05\right]$, and this was unaffected by preexperimental familiarity $\left[F(1,28)=3.35, \eta p^{2}=0.10 p>.077\right]$. Finally, the stimulus type $\mathrm{x}$ pre-experimental familiarity interaction was statistically not meaningful $\left[F(2,28)=0.16, \eta p^{2}=0.01 p>.854\right]$.

\section{II.5. Discussion}

The current study aimed to investigate how pre-experimental familiarity and memory strength affect recognition memory performance. To account for the former, we used pre-experimentally familiar and unfamiliar visual and verbal items. As for the latter, we applied repetition and LOP. The present findings indicate that recognition performance is affected by memory strength. Furthermore, pre-experimental familiarity does not seem to impact old item recognition accuracy. However, the responses to the pre-experimentally familiar figures were faster compared to the abstract ones. Another interesting discovery was that the new abstract figures were identified more accurately than the preexperimentally familiar ones. Furthermore, our results on the hit rates and the discriminability indexes $\left(\mathrm{A}^{\prime}\right)$ confirmed that deep encoding compared to shallow improves visual and verbal memory of both the pre-experimentally familiar and unfamiliar items (Craik, 2002; Craik \& Lockhart, 1972; Craik \& Tulving, 1975; Newell \& Andrews, 2004). 


\section{II.5.1. The Impact of Memory Strength}

As we argued, deeper LOP and repetition are known to overall improve memory and discrimination performance (Craik \& Lockhart, 1972; Craik \& Tulving, 1975; Hintzman \& Curran, 1997; Newell \& Andrews, 2004). Indeed, and as expected, deeper encoding and re-encoding enhanced recognition accuracy and led to faster reactions to the drawn/semantically processed items compared to the studied ones. These factors denote stronger memories (Marzi \& Viggiano, 2010; Wixted \& Stretch, 2004; Yonelinas, 2002).

Moreover, participants could more accurately recognize the new than the weakly embedded studied items. This finding was consistent regardless of pre-experimentally familiarity or the stimulus being visual or verbal. In other words, old/new decisions seem to be less accurate when they concern weakly embedded memories. An explanation could be the absent-mindedness bias, which occurs when less attention and mental resources are devoted to stimulus processing (i.e., shallow encoding), leading to more prominent false recognition or forgetting (Schacter \& Dodson, 2001). In contrast to shallow processing, deeper LOP engages higher levels of attention and other mental resources, which conjointly create multiple memory traces leading to each deeply encoded representation (Craik, 2002). This notion is supported by our findings on the low discriminability indexes of the studied items.

In contrast to the studied stimuli, recognition of the drawn visual material exceeded the correct identification of the corresponding new ones. Accordingly, and in line with previous research (Delhaye, Bastin, Moulin, Besson, \& Barbeau, 2017; Kafkas \& Montaldi, 2014; Kafkas \& Montaldi, 2018), we observed faster reactions to the drawn than the new visual stimuli. However, the results differed slightly for the verbal items since the semantically processed items were recognized as 'old' just as accurately as the new ones were identified as 'new' and the reaction times did not differ. The difference in the recognition 
performance of the verbal and visual items was likely due to differences in LOP of the deep drawing and intermediate semantic encoding task. Previous research has demonstrated that drawing compared to writing (Wammes, Meade, \& Fernandes, 2016) or phonological encoding reaches deeper during processing and establishes stronger memories (Matlin, 2005). Consequently, the drawing task likely improved the encoding and recognition of the visual items more than the rhyming word task did for the verbal stimuli.

In sum, the differential effect of deep and shallow LOP and repetition on recognition performance designates an apparent memory strength effect independently of pre-experimental familiarity. As such, our results help to resolve previous mixed observations on the assumed advantages of novelty over mnemonic benefits of familiarity. Therefore, we argue that stimulus novelty (i.e., no existing memory representation) can provide processing benefits when contrasted with items relying on weak representations. However, when old item memory is strongly embedded, this processing advantage dissipates.

\section{II.5.2. The Role of pre-experimental Familiarity}

Although we argued that pre-experimental stimulus familiarity could be a relevant factor affecting recognition performance, the present findings do not fully support this notion. Our findings on the correct recognition of the drawn and studied visual items indicated that pre-experimental familiarity does not affect the recognition accuracy of the old items. These findings are partly at variance with those reported by Koutstaal, Reddy, Jackson, Prince, Cendan, and Schacter (2003). In this experiment, well-known and abstract figures were first learned incidentally via a shallow encoding task (i.e., rate how much one liked a figure), which was followed by an old/new recognition test. Results revealed that true recognition of well-known figures exceeded that of the abstract. Certainly, their two-phase design was different from our three-phase paradigm. 
Nevertheless, we also could not find any differences in the recognition accuracy of the shallowly encoded figures. Therefore, a plausible explanation could be that Koutstaal, Reddy, Jackson, Prince, Cendan, and Schacter (2003) used more complex three-dimensional figures compared to our two-dimensional line drawings. As such, the abstract figures in our experiment could have been remembered more easily due to less effortful processing. Indeed, Pezdek (1987) reported improved recognition of simple as compared to complex line drawings and argued that the latter likely involves the need to encode and remember more elaborative details, which is more difficult. Thus, it seems that the impact of preexperimental familiarity on old item recognition can be different depending on stimulus complexity or alternatively memory strength as discussed above.

However, the new abstract figures were more accurately identified than the pre-experimentally familiar ones in the current experiment. This could be explained by source confusion (Malmberg, 2008). Thus, the pre-experimentally familiar figures likely had an existing memory representation, which made the participants prone to making more false alarms. On the other hand, the new abstract figures likely did not have any memory representations. Hence, the participants could avoid source confusion. Such a notion aligns with the findings by Koutstaal, Reddy, Jackson, Prince, Cendan, and Schacter (2003). In this recognition memory experiment, meaningless abstract pictures were either labeled with familiar words (e.g., bread) or not. Results revealed that false alarms increased, and accuracy decreased when meaningful labels were used compared to when no labels were applied. Alternatively, it could be that the new abstract figures in the present study were very different from any existing memory. Thus, the distinctiveness of the new abstract figures could improve their correct identification (Dobbins, Kroll, Yonelinas, \& Liu, 1998).

Our findings on the verbal items did not reveal any differences concerning the recognition accuracy. As such, the present results agree with earlier studies suggesting comparable recognition accuracy performance for 
words and non-words (Hintzman, Caulton, \& Curran, 1999; Hintzman \& Curran, 1997). For example, Hintzman and Curran (1997) used a three-phase design to compare the processing of words and non-words. In this experiment, participants first had to study a list of words and non-words. Then, during the instruction phase, the participants were informed that on a subsequent test, they must indicate whether the stimulus is a word or a non-word (lexical decision task) and perform an old/new recognition test. Their findings revealed that recognition of both types of stimuli was comparable. The authors argued that this observation is likely due to comparable stimulus familiarity achieved during learning.

To summarize, when pre-experimental experience exists, separation of internal and external experimental information sources is possible when previously memorized visual or verbal items are judged. However, preexperimental familiarity can lead to decreased recognition performance of visual but not verbal items that do not rely on experimentally induced memory, and thus, are new in the context of an experiment.

\section{II.5.3. Response Times}

Interestingly, participants in our experiment reacted faster to the preexperimentally familiar figures compared to the abstract ones. This observation somewhat corroborates those of Koutstaal, Reddy, Jackson, Prince, Cendan, and Schacter (2003) and supports the notion that processing of pre-experimentally familiar as opposed to unfamiliar abstract figurative material is faster (BellhouseKing \& Standing, 2007; Boucher et al., 2016). The dual-coding theory of picture materials can also provide a plausible explanation (Paivio, 1971, 2007). In this context, it seems likely that the familiar figures were encoded with a pictorial and verbal code. In contrast, it seems less likely that a verbal code was created for the abstract figures since those were meaningless (Voss \& Paller, 2009). Therefore, the connection between the visual and verbal systems could conjointly facilitate 
the activation of the memory trace, which allowed faster discrimination of the well-known figures. Alternatively, this finding corroborates the assumptions of the propositional network theory (Anderson, 1983). According to this theory, retrieval activates cue nodes and the related connections, including the target concepts in long-term memory. Successful retrieval is achieved when the target concept activation exceeds a certain threshold. Thus, it could be that the activation of the pre-experimentally familiar visual memory traces surpassed this threshold faster since their representations were likely used multiple times in the past.

Our findings on the verbal items did not reveal any differences regarding the RT-s. The present results are in contrast to those reported by Perea, Marcet, Vergara-Martinez, and Gomez (2016) and Hintzman and Curran (1997). In the two-block lexical decision experiment by Perea, Marcet, Vergara-Martinez, and Gomez (2016), the processing of words and non-words was investigated. The findings on the RT-s revealed faster reactions in response to the words than the non-words. However, it should be noted that their experimental design differed from the current one considering the lack of a recognition phase. Similarly, Hintzman and Curran (1997) also found that RT-s were faster to the words than the non-words. However, they reduced their analyses to trials with $100<\mathrm{RT}<$ 350 milliseconds and excluded the trials below and above these values. This explains why the present results might be different. Thus, as mentioned above and based on the present results, it seems likely that the experimentally induced stimulus familiarity during memorization was comparable.

In sum, in the context of the RT-s, pre-experimental familiarity affects visual but not verbal memory. This finding could be explained by the dual coding mechanism of pictures and the likely frequent activation of the preexperimentally existing memory traces. However, it could also be that the quicker RT-s related to the familiar than the unfamiliar figures involved unintentional retrieval relying on implicit memory. This type of memory can be 
distinguished from intentional retrieval, referred to as explicit memory (Tulving \& Schacter, 1990), measured in the current study.

In conclusion, the current study suggests that old item recognition of pre-experimentally familiar and unfamiliar visual and verbal materials is comparable. Recognition performance primarily depends on experimentally induced memory strength. As such, we argue that pre-experimental familiarity does neither mitigate nor lead to marked performance differences in overall old visual and verbal item memory but can contribute to faster processing times when the stimuli are visual. Finally, pre-experimental familiarity may worsen correct new visual item identification by increasing false positive answers given to figures that were pre-experimentally familiar to the participants. 


\section{II.6. Appendix: The Effect of Item Proportions: Control Experiment}

\section{II.6.1. Rationale}

A relevant aspect to recognition memory is item proportion (i.e., the proportion of previously memorized and new stimuli) (Poppenk, Kohler, \& Moscovitch, 2010) and response bias (Estes, 2002). The proportion of old and new items can be equal or unequal, which may affect how the participants respond. The bias parameter can shift towards the liberal end (i.e., favoring an 'old' over a 'new' response) when more previously memorized old than new items are presented during the recognition trial (i.e., unequal item proportions) (Estes \& Maddox, 1995). In contrast, a conservative response style can be preferred when the new and old items are more similar. In this case, the participants respond 'new' more often than 'old' (Brown \& Steyvers, 2005). For example, Hirshman (1995) tested different item proportions and manipulated the duration of item presentation using longer and shorter presentation times, inducing strong and weak item memories, respectively. He showed that compared to the weak items, the items with strong memories were recognized better, and their response criterion shifted towards a liberal bias given their higher memorability. Additionally, there were more hits and fewer false alarms in response to the strong than the weak items. Nevertheless, little research has been done on comparing different item proportions regarding the recognition performance of abstract figures and non-words.

In the experiment above (Experiment 1 in Chapter II), we used a recognition paradigm with unequal old and new item proportions, 60 and 30, respectively. Interestingly, an 'old' response bias was detected when reacting to the pre-experimentally familiar figures. However, as mentioned above, $2 \%$ of the responses were missing as the button presses were not recorded. Therefore, we conducted an exploratory control experiment to account for the effects of item 
proportions and their impact on the response bias. In the present study, different ratios of items were presented to two groups of participants (equal proportion group and unequal proportion group). Based on the results of Hirshman (1995), it was expected that a more liberal response style would be used in response to the drawn/semantically processed items than the studied ones. Furthermore, it was anticipated that a more conservative bias would be detected in the equal as opposed to the unequal item proportion group.

\section{II.6.2. Methods}

The Ethical Committee of the Faculty of Psychology and Neuroscience of Maastricht University granted ethical approval for this experiment. Each participant received monetary compensation or research participation credit points. Ethical Approval Code: ERCPN-211_01_08_2019.

\section{II.6.2.1. Participants}

A total of 44 young, healthy participants (13 males) with a mean age of 24 years were recruited by means of advertising. The main inclusion criteria were identical to Experiment 1. One participant was excluded from the analyses because of a technical failure (no button press was recorded). There were two groups: 1) equal item proportions (22 participants) and 2) unequal item proportions (21 participants). The volunteers in the latter group were partly the same as those analyzed in the pre-experimentally unfamiliar group in Experiment 1 .

\section{II.6.2.2. Procedure}

The procedure was identical to Experiment 1, except that we tested the participants with the abstract figures and non-words only and used equal (22 
deeply memorized, 22 shallowly memorized, and 44 new) and unequal old/new item proportions (30 deeply memorized, 30 shallowly memorized and 30 new). We used 22-22-44 instead of 30-30-30 items in the equal proportion group because we wanted to test the effects of item proportion using the same stimulus set as in Experiment 1.

\section{II.6.3. Data Analyses}

The data were analyzed the same way as described in Experiment 1. However, the between-subject variable was item proportion (equal and unequal) instead of pre-experimental familiarity. Furthermore, to investigate the response criterion, non-parametric B" statistics were computed for the drawn/semantically processed and the studied stimuli using Equations (1) (see below) (Stanislaw \& Todorow, 1999). B" varies from -1 to 1 . A value close to 0 indicates that there is no bias, positive values reflect a liberal response criterion (i.e., a tendency to respond 'old' more often than 'new'), and negative values are indicative of a conservative criterion (i.e., an inclination to favor 'new' over 'old' responses) (Stanislaw \& Todorow, 1999).

$$
\mathrm{B}^{\prime \prime}=\operatorname{sign}(H R-F A R) \frac{H R(1-F A R)-F A R(1-F A R)}{H R(1-H R)+F A R(1-F A R)}
$$

$\mathrm{B}^{\prime \prime}$ : response bias, HR: hit rate, FAR: false alarm rate.

\section{II.6.4. Results}

\section{II.6.4.1. Abstract Figures}

The ANOVA investigating the recognition accuracy yielded a significant item proportion $\mathrm{x}$ stimulus type interaction $\left[F(2,68)=4.09, \eta p^{2}=0.17, p<.024\right.$; see Table 1]. However, simple effects analyses did not indicate any differences between the groups for the drawn, studied, and new abstract figures (all 
associated $t$-values $<2.48$, all $p$-values $>$.060). Moreover, stimulus type resulted in a significant main effect $\left[F(2,82)=97.56, \eta p^{2}=0.70, p<.001\right.$; see Table 1]. Posthoc tests revealed that the drawn items were recognized better than the studied $(p<.001)$ and the new figures $(p<.001)$. Additionally, new items were rejected more accurately than the studied items were recognized as old correctly $(p<.001)$. There was no main effect of item proportion $\left[F(1,41)=1.64, \eta p^{2}=0.04, p>.208\right]$.

Table 1. Means (SEMs) of the signal-detection measures of the recognition performance of the abstract figures and non-words according to stimulus type (drawn/semantically processed, studied, and new) and item proportion (equal and unequal).

\begin{tabular}{|c|c|c|c|c|c|}
\hline \multirow[b]{2}{*}{ Stimulus } & \multirow[b]{2}{*}{ Parameters } & \multicolumn{2}{|c|}{ Equal item proportion } & \multicolumn{2}{|c|}{ Unequal item proportion } \\
\hline & & Abstract & Non-words & Abstract & Non-words \\
\hline Type & & Figures & & Figures & \\
\hline Drawn/ & HR & $0.99(0.00) a a, b b$ & $0.85(0.02)^{a a}$ & $0.96(0.01)^{a a, b b}$ & $0.78(0.04)^{a a}$ \\
\hline \multirow{2}{*}{$\begin{array}{l}\text { Semantically } \\
\text { processed }\end{array}$} & $\mathrm{A}^{\prime}$ & $0.89(0.02){ }^{a a}$ & $0.71(0.02)^{a a}$ & $0.88(0.01)^{a a}$ & $0.67(0.02)^{a a}$ \\
\hline & $\mathrm{B}^{\prime \prime}$ & $-0.55(0.18)^{a a}$ & $-0.18(0.22)$ & $-0.62(0.18)^{a a}$ & $-0.05(0.22)$ \\
\hline \multirow[t]{3}{*}{ Studied } & HR & $0.65(0.05)$ & $0.56(0.04)$ & $0.54(0.05)$ & $0.47(0.04)$ \\
\hline & $\mathrm{A}^{\prime}$ & $0.66(0.03)$ & $0.58(0.01)$ & $0.62(0.02)$ & $0.55(0.01)$ \\
\hline & $\mathrm{B}^{\prime \prime}$ & $-1.00(0.00)$ & $-0.64(0.04)$ & $-0.81(0.13)$ & $-0.33(0.21)$ \\
\hline New & CRR & $0.91(0.01)^{a a}$ & $0.79(0.03)^{a a}$ & $0.93(0.01)^{a a}$ & $0.83(0.02)^{a a}$ \\
\hline
\end{tabular}

HR: hit rate, CRR: correct rejection rate, $\mathrm{A}^{\prime}$ : discriminability index. $\mathrm{B}^{\prime \prime}$ : response criterion. Stimulus type effects: different from the studied items: aa $p<.001$, different from the new items: $b b p<.001$.

With respect to the discriminability index $\left(\mathrm{A}^{\prime}\right)$, the mixed ANOVA yielded a main effect of stimulus type $\left[F(1,41)=257.31, \eta p^{2}=0.86, p<.001\right.$; see Table 1]. Post-hoc tests showed that the drawn items were discriminated better than the studied $(p<.001)$. No main effect of item proportion was detected [F (1, $\left.41)=1.33, \eta p^{2}=0.03, p>.256\right]$. There was also no interaction found between the factors $\left[F(1,41)=1.60, \eta p^{2}=0.04, p>.213\right]$. 
Regarding the response criterion $\left(\mathrm{B}^{\prime \prime}\right)$, the ANOVA resulted in a main effect of stimulus type indicating that a more conservative criterion was used in response the studied than the drawn items $\left[F(1,41)=8.07, \eta p^{2}=0.17, p<.007\right.$; see Table 1]. However, there was no main effect of item proportion $[F(1,41)=0.124$, $\left.\eta p^{2}=0.01, p<.727\right]$ and no interaction between the two factors $\left[F(1,41)=1.35, \eta p^{2}\right.$ $=0.03, p<.251]$.

The ANOVA concerning the recognition speed yielded a significant main effect of stimulus type $\left[F(2,40)=125.42, \eta p^{2}=0.86, p<.001\right.$; see Table 2]. Post-hoc tests revealed that the drawn items were recognized faster than the studied $(p<.001)$ and the new ones $(p<.0 .001)$. There was no difference between the studied and new items $(p>.999)$. Neither item proportion nor the stimulus type $\mathrm{x}$ item proportion interaction were found to be statistically meaningful $[F(1$, $41)=0.78, \eta p^{2}=0.02, p>.727 ; F(2,40)=1.48, \eta p^{2}=0.07, p>.240$, respectively].

Table 2. Median reaction times (middle 50\% range; in milliseconds in response to the drawn/semantically processed, studied, and new abstract figures and non-words) and their corresponding first and third interquartile ranges for the equal and unequal item proportion groups.

\begin{tabular}{|c|c|c|c|c|}
\hline & \multicolumn{2}{|c|}{ Equal items proportion } & \multicolumn{2}{|c|}{ Unequal items proportion } \\
\hline \multirow[t]{2}{*}{ Stimulus Type } & Abstract & Non-words & Abstract & Non-words \\
\hline & Figures & & Figures & \\
\hline \multirow{2}{*}{$\begin{array}{l}\text { Drawn/Semantically } \\
\text { processed }\end{array}$} & $658^{a a, b b}$ & 648 & $649 a a, b b$ & 643 \\
\hline & $(599-752)$ & $(599-682)$ & $(612-739)$ & $(619-667)$ \\
\hline \multirow[t]{2}{*}{ Studied } & 747 & 671 & 794 & 642 \\
\hline & $(697-856)$ & $(607-725)$ & $(714-957)$ & $(575-695)$ \\
\hline \multirow[t]{2}{*}{ New } & 778 & 652 & 810 & 699 \\
\hline & $(709-911)$ & $(604-696)$ & (744-918) & $(644-739)$ \\
\hline
\end{tabular}




\section{II.6.4.2. Non-words}

The ANOVA investigating the recognition accuracy including the factors item proportion and stimulus type yielded a main effect of stimulus type, $\left[F(2,40)=53.62, \eta p^{2}=0.73, p<.001\right.$; see Table 1]. Post-hoc tests revealed that the semantically processed non-words were more accurately recognized than the studied ones $(p<.001)$. The new items were identified more accurately than the studied $(p<.001)$. However, no difference was found between the semantically processed and new items $(p>$.999). Item proportion did not result in a significant main effect $\left[F(1,41)=2.18, \eta p^{2}=0.05, p>.148\right]$, and there was no interaction between the factors $\left[F(2,40)=2.36, \eta p^{2}=0.11, p>.108\right]$.

The analyses of the $\mathrm{A}^{\prime}$ scores yielded a main effect of stimulus type indicating that the semantically processed non-words were discriminated better than the studied $\left[F(1,41)=108.66, \eta p^{2}=0.73, p<.001\right.$; see Table 2]. Neither item proportion $\left[F(1,41)=0.02, \eta p^{2}=0.07, p>.083\right]$ nor the interaction between the factors was statistically meaningful $\left[F(1,41)=0.37, \eta p^{2}=0.01, p>.751\right]$.

Regarding the response criterion $\left(\mathrm{B}^{\prime \prime}\right)$, the ANOVA did not reveal any significant differences for the item proportion, stimulus type or the interaction term between these factors [all associated $F$-values $<4.18, \eta p^{2}<0.09, p>.05$ ].

Finally, the ANOVA concerning the reaction times did not yield any significant results [all associated $F$-values $<2.97, \eta p^{2}<0.13, p>.063$ ]. The medians and interquartile ranges are displayed in Table 2.

\section{II.6.5. Brief discussion of the results}

The present experiment investigated the impact of different item proportions (equal and unequal) on the recognition performance involving abstract figures and non-words. In contrast to our expectations, item proportion did not impact the recognition accuracy, reaction times, discriminability $\left(\mathrm{A}^{\prime}\right)$, or 
the response bias $\left(\mathrm{B}^{\prime \prime}\right)$ of the abstract figures or the non-words. Concerning the response bias, despite an overall more conservative response style, participants seemed to favor an 'old' over a 'new' response more when reacting to the drawn/semantically processed than the studied items. Additionally, and as anticipated, it was easier to discriminate the deeply processed and repeated items than the shallowly encoded ones shown only one time. Our findings on the $\mathrm{A}^{\prime}$ and B" parameters align with previous work (Hirshman, 1995; Schacter \& Dodson, 2001).

When using pre-experimentally familiar stimuli, memory load can influence recognition performance. For example, a higher memory load in face recognition resulted in worse memory accuracy in the young and old participants (Lamont, Stewart-Williams, \& Podd, 2005). In this study, the equal group memorized 44 items (drawn and studied) and the unequal group 60 items. Because of the lower memory load, it would have been reasonable to find better memory performance in the equal group. In contrast, there was no difference between the two groups. Thus, item proportion does not seem to influence recognition accuracy when pre-experimentally unfamiliar items are used.

Furthermore, different item proportions can induce a response bias interacting with the memory load effect. For example, in the unequal group, 60 memorized items were contrasted with 30 new ones, and the participants had to answer more often 'old' to give a correct response. In contrast, 44 memorized items had to be compared to an equal number of new stimuli in the equal group. Nevertheless, no differences were found concerning the response bias for either the abstract figures or the non-words. This observation indicates that both groups likely required a similar amount of memory evidence to favor a particular response independently of the memory strength manipulation. Thus, it seems plausible that the pre-experimentally unfamiliar nature of the stimuli might necessitate higher degrees of mnemonic evidence than pre-experimentally familiar items would require. This notion is supported by the negative B" values 
found in both groups, with all stimuli indicating an overall bias reflecting a conservative response style (Dopkins, Sargent, \& Ngo, 2010).

Finally, different levels of recognition load might have a more dominant effect compared to item proportions. Lamont, Stewart-Williams, and Podd (2005) defined recognition load as the total number of items shown during the recognition trial. For face recognition, they found that memory accuracy decreases with increasing recognition load. Concerning the item proportion groups in the present study, the recognition load for the abstract figures was 90 and 88 items, respectively, but no difference in memory performance was found. Of note, we observed a ceiling effect for the abstract figures in the equal item proportion group. In the future, higher levels of memory load and different levels of recognition load should be examined for pre-experimentally unfamiliar stimuli to see whether there are undiscovered effects. In conclusion, it can be said that item proportion does not influence the recognition performance of abstract figures and non-words. 


\section{Chapter:}

EEG Correlates of old/new

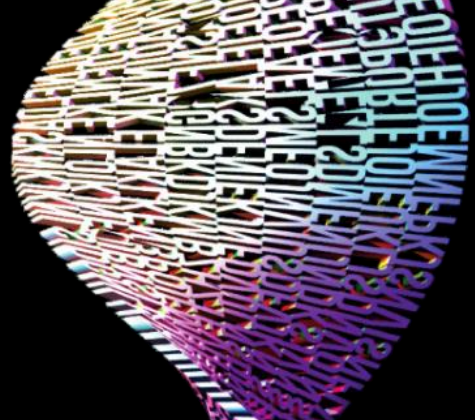

Discrimination Performance involving Abstract Figures and Non-Words

Toth, M.; Sambeth, A.; Blokland, A.

EEG Correlates of Old/New Discrimination

Performance Involving Abstract Figures and Non-Words.

Brain Sci. 2021, 11, 719. https://doi.org/ 10.3390/brainsci11060719 


\section{III.1. Abstract}

The processing of pre-experimentally unfamiliar stimuli such as abstract figures and non-words is poorly understood. Here, we considered the role of memory strength in the discrimination process of such stimuli using a three-phase old/new recognition memory paradigm. Memory strength was manipulated as a function of the Levels of Processing (deep vs. shallow) and repetition. Behavioral results were matched to brain responses using EEG. We found that correct identification of the new abstract figures and non-words was superior to old item recognition when they were merely studied without repetition, but not when they were semantically processed or drawn. EEG results indicated that successful new item identification was marked by a combination of the absence of familiarity (N400) and recollection (P600) for the studied figures. For both the abstract figures and the non-words, the parietal P600 was found to differentiate between the old and new items (late old/new effects). The present study extends current knowledge on the processing of pre-experimentally unfamiliar figurative and verbal stimuli by showing that their discrimination depends on experimentally induced memory strength and that the underlying brain processes differ. Nevertheless, the P600, similarly to pre-experimentally familiar figures and words, likely reflects improved recognition memory of meaningless pictorial and verbal items.

Keywords: discrimination; recognition memory; EEG old/new effects; abstract figures; non-words 


\section{III.2. Introduction}

An efficient memory system requires the ability to detect and incorporate new information and to readily retrieve familiar information (Donaldson, 1992; Kafkas \& Montaldi, 2014; Kafkas \& Montaldi, 2018; Kumaran \& Maguire, 2007). Information-processing theories define recognition as a predicament of discrimination between what is known (familiar) and what is not known (new) (Benjamin, Diaz, \& Wee, 2009; Stanislaw \& Todorow, 1999; Verde \& Rotello, 2007; Yonelinas, 2002). Discrimination performance is typically tested with paradigms that assess recognition memory. In such paradigms, participants are required to recognize previously studied stimuli (e.g., typically well-known and meaningful figures and words) as old correctly and to identify previously not seen items as new (Malmberg, 2008). The recognition/discrimination process generates a cue, which induces either a sense of familiarity or unfamiliarity (novelty) (Yonelinas, 2002; Yonelinas, Kroll, Dobbins, Lazzara, \& Knight, 1998). The decision process determines how we respond: whether a stimulus is judged as old or new.

While the processing of meaningful and pre-experimentally familiar stimuli is well-researched, less is known about how the brain recognizes and discriminates pre-experimentally unfamiliar and meaningless items. These stimuli can be characterized as previously not seen combinations of simple shapes (abstract figures) and letters (non-words). Knowing how the brain processes and discriminates such stimuli can significantly improve our understanding of visual, lexical, and orthographic memory processing while contributing to establishing relevant cognitive models such as age-related memory impairment. As a result, research in this field could aid patients with orthographic dyslexia, lexical deficiencies, or memory impairment, especially if behavioral and EEG results can be combined with brain-computer interface (BCI) approaches (Paszkiel, 2017, 2020; Paszkiel \& Szpulak, 2018). Therefore, the 
current work investigated the behavioral and electrophysiological processing of pre-experimentally unfamiliar figurative and verbal items.

Behavioral data indicate that old/new discrimination at least partly depends on the assessment of the strength of a particular memory. Memory strength is assumed to vary on a continuum ranging from weak to strong, which underlies the subjective perception of stimulus familiarity (Wixted \& Stretch, 2004; Yonelinas, 2002). In other words, the stronger the stimulus memory, the more robust the sense of familiarity appears, and the more likely it is that a stimulus is judged 'old' rather than 'new'.

Previous studies have shown that memory strength, the ease by which a particular memory can be recalled or recognized, can effectively be manipulated as a function of repetition (Verde \& Rotello, 2007) or by using Levels of Processing (LOP) (Gardiner, 1988). Repetition is known to strengthen memory by increasing the subjective sense of familiarity resulting from re-encoding of a particular memory trace (Hintzman \& Curran, 1997; Ranganath \& Rainer, 2003). The LOP theory predicts that deep (e.g., meaning-extraction, pattern recognition, activation of prior knowledge) and intermediate processing (e.g., phonetics) lead to superior and faster retrieval when compared to shallow processing (e.g., perceptual analyses, rehearsal) (Craik, 2002; Craik \& Lockhart, 1972; Craik \& Tulving, 1975; Newell \& Andrews, 2004). Such findings are supported by brain imaging studies that have found more positive late parietal P600 amplitudes for the deeply- over the shallowly-encoded stimuli (Norman, Tepe, Nyhus, \& Curran, 2008; Nyhus \& Curran, 2009), which corroborates an episodic memoryrelated function of this evoked response potential (ERP) (Rugg \& Curran, 2007).

For example, Harris, Cutmore, O'Gorman, Finnigan, and Shum (2013) used an auditory word recognition task in which half of the trials made use of shallow encoding (i.e., determine if an auditory word comprised long vowels) and the other half used deep encoding (i.e., incorporate an auditory word into a meaningful sentence). According to their findings, not only were the deeply 
encoded words more accurately recognized than the shallow, but the related P600 amplitudes of the deeply encoded words were also larger compared to the shallow. Moreover, previous research has shown that mnemonics such as verbal (e.g., rhyming), motor (e.g., drawing), or visual (e.g., imagining) techniques strengthen memory storage and improve subsequent retrieval as a result of deeper processing (Hulstijn, 1997; Jones-Gotman, 1986; Paivio \& Desrochers, 1981; Solso, 1995).

In the present study, we considered the role of memory strength in the discrimination process of meaningless abstract figures and non-words using a three-phase old/new recognition memory paradigm. We chose to include both figurative and verbal stimuli since these are known to be processed differently (Kim, Yoon, \& Park, 2004). For instance, pictures are generally remembered better than words (i.e., picture superiority effect), since the former in contrast to the latter, are assumed to be encoded with dual (image and verbal) as opposed to singular labels (verbal), respectively (Paivio, 1971, 2007; Rajaram, 1996). In the current experiment, we first familiarized the stimuli using mnemonics in order to induce deep processing (deep memorization): we asked the participants to redraw the abstract figures and to come up with existing rhyme words for the non-words (semantic encoding). In the second phase, participants were asked to merely study the stimuli (shallow memorization). Here, the previously deeply encoded items were shown again in combination with some new items. Finally, an old/new recognition test was applied in which stimuli from the first and second phases were intermixed with new ones. We assessed both recognition accuracy and speed.

According to our knowledge, this is the first study examining the impact of memory strength on the discrimination performance of pre-experimentally unfamiliar items using such a design. It is known that identifying something as new compared to it being old usually takes longer than old item recognition (Delhaye, Bastin, Moulin, Besson, \& Barbeau, 2017; Kafkas \& Montaldi, 2014; 
Kafkas \& Montaldi, 2018). Therefore, we assumed that the recognition of the drawn/semantically processed and studied items would be faster compared to the correct identification of the new items. Moreover, we anticipated that the drawn/semantically processed stimuli would be recognized more accurately and faster than the studied ones.

To match the possible effects of new and old item recognition with brain processes, we measured electrophysiological responses during retrieval. Four ERPs of interest were the N200, P300, N400, and P600. Recent research has shown how these different ERPs can be helpful in the context of memory research and BCIs. For example, the P300 has been used to discriminate familiar from unfamiliar faces (Marcel \& Milla 'n, 2007). Based on the N400, it was possible to infer semantic relatedness of verbal stimuli (Dijkstra, Farquhar, \& Desain, 2019; Xie et al., 2018), and with the P600, it was possible to differentiate between target and lure audiovisual number stimuli (Xie et al., 2018).

EEG data on the processing of abstract images are scarce and not well understood. For instance, identification of unusual stimuli in oddball tasks typically elicits larger N200 and P300 amplitudes (Patel \& Azzam, 2005). Interestingly, O'Hare and Goodwin (2018) found that abstract artwork images elicited larger P300 amplitudes than natural ones. At the same time, they failed to detect differences for the N200. Beisteiner et al. (1996) failed to find differences in the P300 amplitude during the recognition of old and new abstract geometric images. Later old/new correlates typically elicit the so-called early (N400) and late (P600) old/new effects, respectively (Rugg \& Curran, 2007). The frontocentral N400 is postulated to represent the effective use of familiarity to discriminate between old and new items (Curran \& Hancock, 2007; Friedman \& Johnson, 2000; Rugg \& Curran, 2007). As such, early old/new effects are seen as more negative amplitudes for new stimuli that were correctly endorsed as 'new' compared to previously seen old stimuli that were recognized as 'old' (Curran \& Hancock, 2007; Friedman \& Johnson, 2000; Rugg \& Curran, 2007). Applying this 
to the current paradigm, more negative amplitudes of the correctly detected new compared to old images would reflect optimal discrimination of these stimuli. The parietal late old/new effect (P600) typically entails larger amplitudes of the pre-exposed compared to the not pre-exposed items (Friedman \& Johnson, 2000; Rugg \& Curran, 2007; Yonelinas, 2002). At the same time, this reflects improved memory performance (Finnigan, Humphreys, Dennis, \& Geffen, 2002; Kim, Yoon, \& Park, 2004). Therefore, we anticipated a more positive late old/new effect for the drawn and repeated figures that relied on strong memories compared to the new ones. Likewise, we expected to find late old/new effects for the studied figures that relied on weak memories.

Electrophysiological processing of non-words is somewhat better understood. For instance, whereas words affect the N400, non-words influence the P300 (Otten, Sveen, \& Quayle, 2007; van Dinteren, Arns, Jongsma, \& Kessels, 2014). The amplitude of the P300 is thought to index context updating as a result of initiated input comparisons to existing representations (Patel \& Azzam, 2005; Polich, 2007; van Dinteren, Arns, Jongsma, \& Kessels, 2014). Its amplitude enhances whether a stimulus does not fit or weakly matches any existing representation (Polich, 2007). Additionally, according to the context-updating hypothesis, increased N200 and decreased P300 amplitudes are indicative of a match to an existing memory template, and the opposite is true if the match is vague (Patel \& Azzam, 2005). Therefore, semantically processed stimuli should elicit the former pattern resulting from deeper LOP and repetition. In contrast, the studied stimuli should show the latter pattern as a result of shallow LOP without repetition. Considering that the P600 has been linked to successful old/new item discrimination and has been found with words (Smith \& Guster, 1993; Van Petten \& Senkfor, 1995) and non-words (Fjell, Walhovd, \& Reinvang, 2005), we assumed we would detect late parietal old/new effects. This was expected to show in a larger peak amplitude for the correctly recognized old than new non-words. 
Finally, in terms of the LOP manipulation, we expected to detect more positive amplitudes for the deeply- over the shallowly memorized abstract figures and non-words (Norman, Tepe, Nyhus, \& Curran, 2008; Nyhus \& Curran, 2009).

\section{III.3. Materials and Methods}

The Ethical Committee of the Faculty of Psychology and Neuroscience of Maastricht University granted ethical approval for this experiment. Each participant received monetary compensation or research participation credit points. On average, the experiment took 1.5 hour/test-session (Ethical Approval Code: ECP13_02_2012).

\section{III.3.1. Participants}

A total of 22 young, healthy participants (eight males) with a mean age of 24 years were recruited by means of advertising. The main inclusion criteria were age (18-30 years) and being fluent in the English or Dutch languages. One participant was excluded from the behavioral analyses because of a technical failure.

\section{III.3.2. Procedure}

After signing an informed consent, participants were admitted to the study. Prior to starting the experiment, each participant filled in a demographic questionnaire including information about sex, age, and handedness. At this point, the EEG caps were installed. During the test, stimuli were presented via a computer screen, and participants had to respond on two keys of a response pad. Recognition accuracies and reaction times were recorded. 
A memory paradigm with abstract figures and non-words was applied in separate tests (see Figure 1 for an example of the stimuli used). Every participant performed each test phase first with the abstract figures and then with the non-words in order to minimize verbalization of the figurative stimuli. The experiment consisted of three phases (see Figure 2). In phase 1 (deep memorization leading to 'strong' memory), participants were familiarized with a series of 30 monosyllabic abstract figures or non-words in separate tests (list 1: L1). Participants were asked to manually redraw the abstract figures on an answer sheet in order to induce deep LOP. They had to mention existing English or Dutch rhyming words for each non-word to induce intermediate LOP. Stimuli were presented for $1 \mathrm{~s}$, and the participants were given $14 \mathrm{~s}$ to execute the mnemonic encoding task. If they were ready earlier, they could press a button, and $2 \mathrm{~s}$ later, the next stimulus appeared. Stimuli were extracted from previous studies (Glosser, Friedman, Grugan, Lee, \& Grossman, 1998; Redoblado, Grayson, \& Miller, 2003; Seidenberg, Plaut, Petersen, McClelland, \& McRae, 1994).

\begin{tabular}{|l|l|l|l|}
\hline \multicolumn{3}{|c|}{ Abstract Figures } & \multicolumn{3}{c|}{ Non-Words } \\
\hline & & \\
\hline
\end{tabular}

Figure 1. Examples of the stimuli used. 


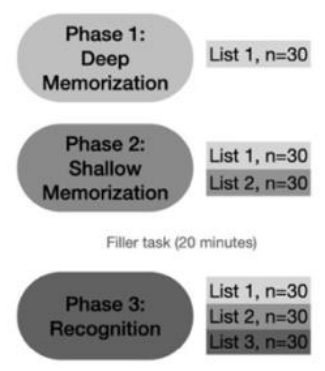

Figure 2. Schematic overview of the experimental design. Phase 1: deep memorization with the preexperimentally unfamiliar abstract figures and non-words in separate tests using a mnemonic encoding task (redrawing the abstract figures and mentioning rhyming words for the non-words). The 30 stimuli used here form List 1 (drawn/semantically processed stimuli). Phase 2: shallow memorization with the instruction to remember as many stimuli as possible. This phase contained items from List 1 and 30 new ones (List 2, studied stimuli). Phase 3: recognition of the stimuli including List 1, List 2, and 30 new (List 3). n: number of stimuli presented.

During phase 2 (shallow memorization leading to 'weak' memory), participants were instructed to remember as many stimuli as possible. In this phase, 60 stimuli (abstract figures or non-words) were used: 30 stimuli from L1 were randomly mixed with 30 new ones (L2). All stimuli were shown for $1 \mathrm{~s}$ with an inter-stimulus interval (ISI) of $2 \mathrm{~s}$.

During phase 3, participants were asked to decide if they had seen the presented stimulus in the previous series (L1 and L2) or whether the stimulus was new to them (L3: new, $n=30$ ). The 90 non-words or abstract figures were presented for a duration of $1 \mathrm{~s}$, or less in the case of faster button press; the ISI was $2.5 \mathrm{~s}$. Participants had to press the corresponding buttons ('old' for L1 and 
L2, or 'new' for L3 stimuli) on a response box as quickly and accurately as possible.

A filler paper-and-pencil task of $10 \mathrm{~min}$ and another non-verbal task were given between phase 2 and 3 . The filler task consisted of the localization of number sequences, vertically or horizontally placed within a field of numbers (10 min). The other task consisted of watching a silent cartoon while auditory stimuli were presented $(10 \mathrm{~min})$.

EEG was recorded simultaneously with the behavioral testing. Recordings were carried out with a standard EEG apparatus using 32 electrodes, placed according to the 10/20 standard international placement of electrodes. Eye movements were monitored through electrooculograms, with electrodes placed above and below the left eye as well as at the corner of the left and right eyes. The ground electrode was placed at FPz. Reference electrodes were applied at the left and right mastoid bones. Data were sampled at $512 \mathrm{~Hz}$ and filtered between 0.05 and $100 \mathrm{~Hz}$ during acquisition. After the recording, data were pre-processed using Brain Vision Analyzer 2 (Brain Products, Munich) including filtering (1-30 $\mathrm{Hz}$ ), ocular correction, segmentation, baseline correction (-100 to $0 \mathrm{~ms})$, artifact rejection (exclusion of trials if signal exceeded +100 or $-100 \mu \mathrm{V}$ ), and calculation of averages and grand averages by averaging the responses between $100 \mathrm{~ms}$ before and $1000 \mathrm{~ms}$ after stimulus onset for the correct responses of each stimulus type separately. Sequentially, the data were clustered into frontal (F3, F4, and Fz), central (C3, C4, and Cz), and posterior (P3, P4, and Pz) sections (Rugg \& Curran, 2007). Based on visual inspection of the grand averages, the N200, P300, N400, and P600 peak amplitudes and latencies were determined using the time windows presented in Table 1. Next, as suggested by Luck (2005), we applied the local peak amplitude detection method available in Brain Vision Analyzer 2. 
Table 1. Overview of the time windows of the analyzed ERP components during recognition (in milliseconds).

\begin{tabular}{ccccc}
\hline $\begin{array}{c}\text { Stimulus } \\
\text { Category/ERP }\end{array}$ & N200 & P300 & N400 & P600 \\
\hline Abstract Figures & $200-320$ & $300-420$ & $400-500$ & $500-700$ \\
\hline Non-words & $150-250$ & $250-400$ & $400-500$ & $500-700$ \\
\hline
\end{tabular}

\section{III.3.3. Data Analysis}

Before analysis, all data were evaluated for having normal distribution and homogeneity of variance. Additionally, raw data were checked for outliers. Outlier values were replaced with their regression estimates produced by the missing value analyses (IBM SPSS Statistics for Macintosh, Version 27.0. Armonk, NY: IBM Corporation). Additionally, due to technical issues, 1-2 responses per participant were missing (e.g., the button press was not recorded). In these cases, values were replaced with their regression estimates. Effect sizes are reported based on partial eta-squared ( $\eta p 2)$ data. Furthermore, Mauchly's test of sphericity was applied. In case the assumption of sphericity was violated, a GreenhouseGeisser correction was used. In all cases, degrees of freedom of assumed sphericity were reported. Post-hoc comparisons and simple effects were investigated using paired-samples t-tests, applying adjustments for multiple comparisons; the observed p-values were multiplied by the number of comparisons, which was tested against the set significance level of 0.05 .

For the behavioral data, signal detection theory (SDT) was applied in order to investigate the discrimination performance (Benjamin \& Bawa, 2004; Benjamin, Diaz, \& Wee, 2009; Stanislaw \& Todorow, 1999; Verde \& Rotello, 2007). Discrimination accuracy was defined as the ability to distinguish the different types of stimuli (drawn/semantically processed, studied, and new). Correct 
responses included an 'old' response to the drawn/semantically processed items, and the studied stimuli, and a 'new' response to the new items. Incorrect responses involved a 'new' response to the drawn/semantically processed items and the studied stimuli and an 'old' response to the new stimuli. See Table 2 for an overview.

Table 2. Overview of the different types of responses as a function of stimulus type.

\begin{tabular}{ccc}
\hline & Stimulus Type & Response \\
\hline Hit (H) & $\begin{array}{c}\text { Drawn or semantically } \\
\text { processed/Studied }\end{array}$ & 'Old' \\
\hline Miss (M) & $\begin{array}{c}\text { Drawn or semantically } \\
\text { processed/Studied }\end{array}$ & 'New' \\
\hline Correct Rejection (CR) & New & 'New' \\
\hline False Alarm (FA) & New & H/(H + M) \\
\hline Hit Rate (HR) & Drawn or semantically \\
processed/Studied & New & $\mathrm{CR} /(\mathrm{CR}+\mathrm{FA})$ \\
\hline Correct Rejection Rate (CRR)
\end{tabular}

Given the memory strength manipulation in the current design (deep memorization, shallow memorization, and recognition), the correct response rates, being hit rates (HR) for the drawn/semantically processed, and the studied items and correct rejection rates (CRR) for the new, were used to evaluate the discrimination accuracy. Furthermore, in order to investigate discriminability, non-parametric $\mathrm{A}^{\prime}$ statistics were computed for the drawn/semantically processed and the studied stimuli using Equations (1) or (2) (see below) (Snodgrass \& Corwin, 1988; Stanislaw \& Todorow, 1999). A' varies from 0 to 1, with 0.5 indicating chance performance. Higher values are indicative of improved performance (Snodgrass \& Corwin, 1988; Stanislaw \& Todorow, 1999). 


$$
\begin{aligned}
& \mathrm{A}^{\prime}=0.5+\frac{(H R-F A R)(1+H R-F A R)}{4 H R(1-F A R)}, \text { if } H R \geq F A R \\
& \mathrm{~A}^{\prime}=0.5-\frac{(H R-F A R)(1+H R-F A R)}{4 H R(1-F A R)}, \text { if } H R<F A R
\end{aligned}
$$

$\mathrm{A}^{\prime}$ : discriminability index, HR: hit rate, FAR: false alarm rate.

During recognition, the a priori probabilities of old and new items and the quality of the match between a test item and the memory for studied items can influence the bias parameter $[6,45]$. Such a model does not fit the current paradigm due to the memory strength manipulation used and the equivalent proportion and intended comparison of the strong $(n=30)$, weak $(n=30)$, and new items $(n=30)$ [44]. After all, the final proportion of 'old' and 'new' responses was 2:1. Therefore, we calculated the total amount of 'old' $(\mathrm{H}+\mathrm{FA})$ and 'new' $(\mathrm{M}$ $+\mathrm{CR})$ responses given by the participants. This was done to examine whether there was a preference for either the 'old' or 'new' responses. Results were compared using paired samples t-tests with Bonferroni corrections.

RT data of the hits were also evaluated. To be able to use parametric tests, RT-s were transformed into $|\log (1 / \mathrm{RT})|$ to obtain a normal distribution of the data [46]. Moreover, the median RT data are reported as central tendency parameters, together with the corresponding first and third interquartile ranges [47].

Statistical analysis was conducted using SPSS 27.0. A repeated-measures analysis of variance (ANOVA) was used to investigate the discrimination accuracy scores and RT-s for the different stimuli in the different categories as assessed in phase 3. The within-subject variables were stimulus category (abstract figures and non-words) and stimulus type (drawn/semantically processed, studied, and new items). 
For the EEG analysis, a within-subjects repeated-measures analysis of variance (ANOVA) was conducted to investigate the amplitude and latency data during recognition (phase 3). The model included the following factors within each stimulus category: stimulus type with three levels (drawn/semantically processed, studied, and new items) and location with three levels (frontal, central, and parietal). Since the aim of the study was to investigate discrimination performance, the main effects and interactions involving stimulus type (amplitudes and latency data) are described in detail.

\section{III.4. Results}

\section{III.4.1. Behavioral}

Although there was an unequal number of old-responses over newresponses (2:1), we found that there was no response bias (see Table 3). The mean signal-detection parameter estimates are displayed in Table 4.

Table 3. The total number of old and new responses during the recognition test. Data represent the means (SEM) of the total 'old' and 'new' responses and the corresponding \% compared to the 90 items/stimulus category (abstract figures and non-words), and the t-statistics.

\begin{tabular}{|c|c|c|c|c|}
\hline \multirow[b]{2}{*}{ Old responses } & \multicolumn{2}{|c|}{ Abstract Figures } & \multicolumn{2}{|c|}{ Non-Words } \\
\hline & $47.24(2.04)$ & $52 \%$ & $43.10(1.95)$ & $48 \%$ \\
\hline New responses & $42.10(2.10)$ & $47 \%$ & $46.52(1.97)$ & $52 \%$ \\
\hline $\begin{array}{c}\text { Paired samples t- } \\
\text { test }\end{array}$ & \multicolumn{2}{|c|}{$t(20)=1.25, p>.450$} & \multicolumn{2}{|c|}{$t(20)=0.88, p>.784$} \\
\hline
\end{tabular}




\section{III.4.1.1. Abstract Figures}

With respect to the accuracy scores (HR and CRR) of the abstract figures, the ANOVA yielded a significant main effect of stimulus type $\left[F(2.40)=70.24, \eta p^{2}\right.$ $=0.78, p<.001$; see Figure 3A and Table 4]. Post-hoc tests revealed that the drawn stimuli were recognized more accurately than the studied $(p<.001)$ and the new $(p<.027)$. Additionally, more new stimuli were correctly identified compared to the studied items $(p<.001)$.

A

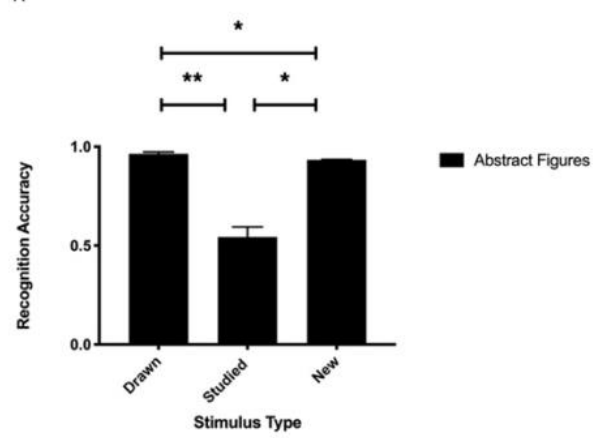

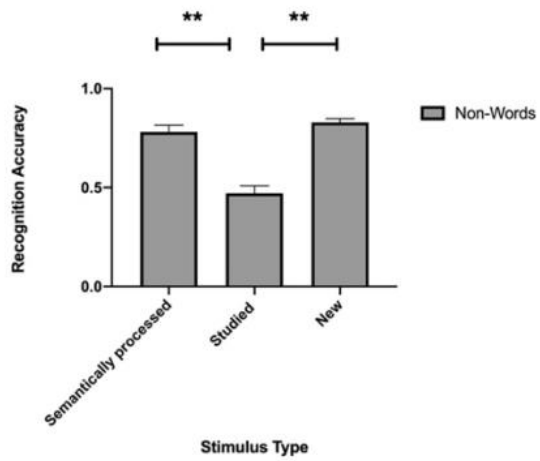

Figure 3. Recognition accuracy performance of the abstract figures (A) and the non-words (B) according to stimulus type. The bars represent the means of the hit rates of the drawn/semantically processed and the studied items, and the means of the correct rejection rates for the new items. Stimulus type effects: **: $p<.001,{ }^{*}: p<.05$ 
Table 4. Means (SEMs) of the signal-detection measures of the recognition performance of the abstract figures and non-words according to stimulus type (drawn/semantically processed, studied, and new).

\begin{tabular}{cccc}
\hline Stimulus Type & Parameters & Abstract Figures & Non-Words \\
\hline $\begin{array}{c}\text { Drawn/Semantically } \\
\text { processed }\end{array}$ & HR & $0.96(0.01)^{* *, *}$ & $0.78(0.04)^{* *}$ \\
\cline { 2 - 4 } & $\mathrm{A}^{\prime}$ & $0.88(0.01)^{* *}$ & $0.67(0.02)^{* *}$ \\
\hline \multirow{2}{*}{\begin{tabular}{c} 
Studied \\
\cline { 2 - 4 }
\end{tabular}} & HR & $0.54(0.05)$ & $0.47(0.04)$ \\
\hline New & $\mathrm{A}^{\prime}$ & $0.62(0.02)$ & $0.55(0.01)$ \\
\hline
\end{tabular}

HR: hit rate, CRR: correct rejection rate, $\mathrm{A}^{\prime}$ : discriminability index. Different from studied: ${ }^{* *}: p<.001$, difference between the drawn and new items: \#: $p<.05$.

The analyses performed on the $\mathrm{A}^{\prime}$ scores revealed that the drawn abstract figures resulted in improved discriminability compared to the studied $\left[F(1.20)=278.10, \eta p^{2}=0.93, p<.001\right.$; see Table 4].

When analyzing the reaction time performance in the session with the abstract figures, a main effect of stimulus type was found $\left[F(2.40)=36.68, \eta p^{2}=\right.$ $0.65, p<.001$; see Table 5]. Post-hoc tests showed that response times were significantly faster to the drawn stimuli compared to the studied $(p<.001)$ and new items $(p<.001)$. No such difference was found between the studied and new abstract figures $(p>.999)$. 
Table 5. Median reaction times (middle 50\% range; in milliseconds in response to the drawn/semantically processed, studied, and new abstract figures and non-words) and their corresponding first and third interquartile ranges.

\begin{tabular}{ccc}
\hline Stimulus Type & Abstract Figures & Non-Words \\
\hline Drawn/Semantically processed & $649(612-739)$ & 643 (619-667) \\
\hline Studied & $794^{* *}(714-957)$ & $642^{*}(575-695)$ \\
\hline New & $810^{* *}(744-918)$ & $699(644-739)$ \\
\hline
\end{tabular}

Different from drawn/semantically processed (stimulus type effects): different from drawn: ${ }^{* *}: p<$ .001 , different from studied: \#: $p<.05$

\section{III.4.1.2. Non-Words}

When investigating the accuracy performance (HR and CRR) of the nonwords, the analyses revealed a significant main effect of stimulus type $[F(2.19)=$ 31.07, $\eta p^{2}=0.77, p<.001$; see Figure 3B and Table 4]. Post-hoc tests revealed that the semantically processed stimuli were recognized more accurately than the studied $(p<.001)$. Additionally, more new stimuli were correctly identified compared to the studied $(p<.001)$. No such difference was found between the semantically processed and new non-words $(p>.849)$.

The analyses of the $\mathrm{A}^{\prime}$ scores revealed that it was easier to discriminate the semantically processed non-words than the studied $\left[F(1.20)=40.05, \eta p^{2}=\right.$ $0.67, p<.001$; see Table 4].

As for the reaction times, stimulus type yielded a significant main effect $\left[F(2.19)=6.45, \eta p^{2}=0.40, p<.007\right.$; see Table 5]. Post-hoc tests showed that response times were significantly faster to the studied than the new non-words $(p<.015)$. No such difference was found between the semantically processed and new items $(p>.069)$, and the semantically processed and studied non-words $(p>$ .999). 
III.4.2. EEG

\section{III.4.2.1. Abstract Figures}

\section{- $\quad$ N200}

The analyses revealed a significant stimulus type $\times$ location interaction $\left[F(4.84)=3.98, \eta p^{2}=0.16, p<.014\right.$; see Figure 4 A-C $]$. Simple effects analyses showed that the amplitude related to the studied abstract figures was more negative than that related to the drawn figures at the posterior location $(p<.039)$. The rest of the combinations were found to be insignificant (all $p$-values $>.105$ ). Stimulus type did not reveal a main effect $\left[F(2.20)=1.31, \eta p^{2}=0.12, p>.292\right]$. The latency of the N200 was not affected by stimulus type or location [all associated F-values $<1.85, \eta p^{2}$-values $<0.17, p$-values $\left.>.183\right]$.
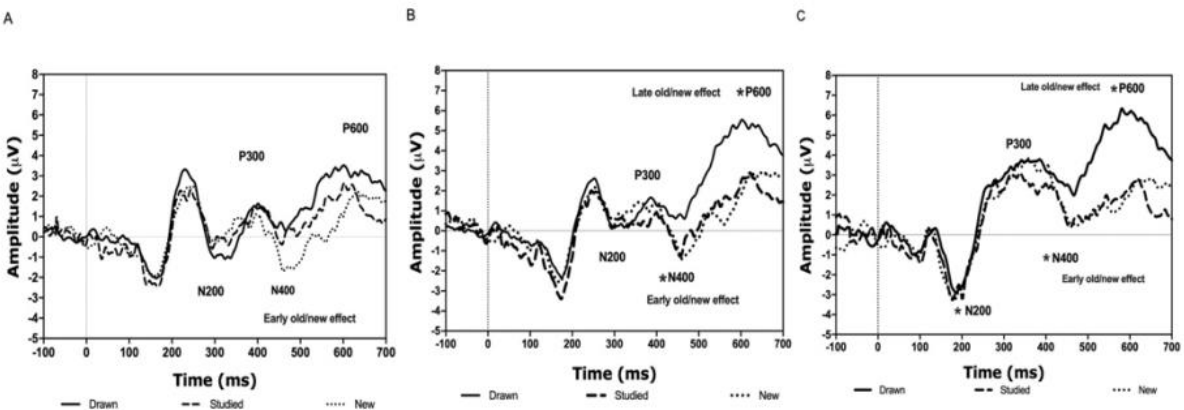

Figure 4. Grand averages across participants for the N200, P300, N400 and P600 during the recognition of the abstract figures according to stimulus type at the frontal (A), central (B), and posterior (C) electrode location clusters. Stimulus type effects: * $p<.05$

\section{- $\quad$ P300}

When analyzing the amplitude data of the P300, a significant stimulus type $x$ location interaction was detected $\left[F(4.84)=5.49, \eta p^{2}=0.21, p<.003\right.$; see Figure 4A-C]. However, simple effects analyses did not reveal any significant 
differences (all $p$-values $>$.168). Stimulus type did not reveal a main effect $[F(2.20)$ $\left.=0.74, \eta p^{2}=0.10, p>.492\right]$.

As for the latency data, neither stimulus type nor the interaction between stimulus type and location was statistically meaningful [all associated $F$-values < $1.42, \eta p^{2}$-values $<0.24, p$-values $\left.>.267\right]$.

\section{- $\quad \mathrm{N} 400$}

According to the analyses, the stimulus type $\mathrm{x}$ location interaction was significant $\left[F(4.84)=6.93, \eta p^{2}=0.25, p<.001\right.$; see Figure $4 \mathrm{~A}-\mathrm{C}$ and Figure 5A]. Simple effects analyses revealed that the amplitude related to the new abstract figures was more negative than that representing the drawn items at the frontal $(p<.003)$, central $(p<.003)$, and posterior locations $(p<.003)$. Additionally, the N400 amplitude related to the studied abstract figures was more negative compared to the drawn at the central $(p<.001)$ and posterior $(p<.001)$ but not the frontal location $(p>.999)$. The rest of the combinations were not significant (all $p$ values $>$.999).

As for the latency, neither the interaction stimulus type $\mathrm{x}$ location nor stimulus type was significant [all associated $F$-values $<2.70, \eta p^{2}$-values $<0.38, p$ values $>.064]$. 

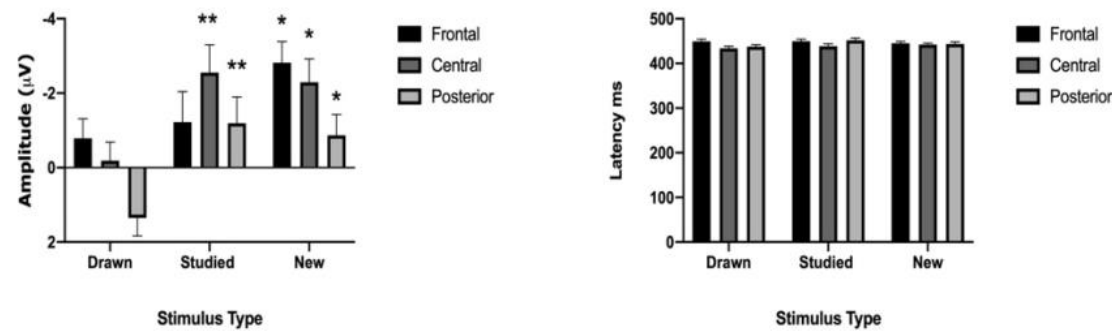

C

D
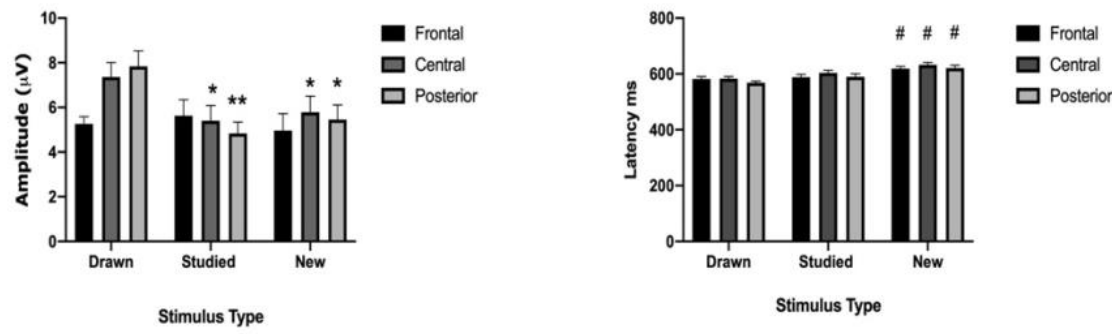

Figure 5. Means of the N400 amplitude (A) and latency (B), and the P600 amplitude (C) and latency (D) during the recognition of the abstract figures. Stimulus type effects: different from the drawn items: ${ }^{* *}: p<.001,{ }^{*}: p<.05$, different from the studied items: \#: $p<.05$

- $\quad$ P600

The analyses showed a significant stimulus type $\times$ location interaction $\left[F(4.84)=13.68, \eta p^{2}=0.40, p<.001\right.$; see Figures $4 \mathrm{~A}-\mathrm{C}$ and $\left.5 \mathrm{C}\right]$. Simple effects analyses revealed that the amplitude related to the drawn abstract figures was larger than that of the studied at the central $(p<.003)$ and posterior locations $(p<$ .003). Additionally, the P600 in response to the drawn items was larger compared to the new at the central $(p<.033)$ and posterior $(p<.006)$ locations. No other differences were detected (all $p$-values $>.968$ ).

As for the latency, there was a significant main effect of stimulus type $\left[F(2,20)=7.47, \eta p^{2}=0.43, p<.004\right.$; see Figures $4 \mathrm{~A}-\mathrm{C}$ and $\left.5 \mathrm{D}\right]$. Post-hoc tests 
showed that the P600 latency related to the new items was shorter compared to the studied $(p<.018)$. No further differences were found (all associated $p$-values $>$.100). The interaction between stimulus type and location was not significant $\left[F(4.84)=0.67, \eta p^{2}=0.03, p>.546\right]$.

\section{III.4.2.2. Non-Words}

- $\quad$ N200

The analyses did not reveal any significant effects for the amplitudes [all associated $F$-values $<2.33, \eta p^{2}<0.19, p>.123$ ] or the latencies [all associated $F$ values $<2.42, \eta p^{2}$-values $<0.20, p$-values $\left.>.114\right]$.

\section{- $\quad$ P300}

The amplitudes were dissimilar for the different stimulus types $[F(2.42)$ $\left.=4.44, \eta p^{2}=0.17, p<.029\right]$. Post-hoc tests revealed that the amplitude related to the studied non-words were marginally larger than those related to the new ( $p=$ 0.053). No further statistically meaningful differences were found (all $p$-values > .165). The stimulus type $\mathrm{x}$ location interaction was not significant $[F(4.84)=1.34$, $\left.\eta p^{2}=0.06, p>.268\right]$.

The latency analyses revealed a significant effect of stimulus type $\left[F(2.42)=9.58, \eta p^{2}=0.31, p<.001\right.$; see Figure $\left.6 \mathrm{~A}-\mathrm{C}\right]$. Post-hoc tests revealed that the P300 in response to the semantically processed stimuli peaked later compared to the studied $(p<.017)$ and the new ones $(p<.006)$. No such difference was found between the studied and semantically processed non-words $(p>.282)$. Finally, neither stimulus type nor the interaction term stimulus type $\mathrm{x}$ location was significant [all associated $F$-values $<1.22, \eta p^{2}$-values < $0.11, p$-values $>.317$ ] 
- $\quad$ N400

The analyses did not reveal any significant effects for the amplitudes [all associated $F$-values $<2.65, \eta p^{2}<0.12, p>.099$ ] or the latencies [all associated $F$ values $<1.41, \eta p^{2}$-values $<0.06, p$-values $\left.>.250\right]$.

\section{- $\quad$ P600}

The analyses showed a significant stimulus type $\mathrm{x}$ location interaction $\left[F(4.84)=8.10, \eta p^{2}=0.28, p<.001\right.$; see Figures $6 \mathrm{~A}-\mathrm{C}$ and $\left.7 \mathrm{~A}\right]$. Simple effects analyses revealed that the P600 amplitude related to the new non-words was smaller than that related to the semantically processed $(p<.009)$ and the studied non-words $(p<.042)$ at the posterior location. No other differences were detected (all $p$-values $>.258)$. Stimulus type did not affect the amplitudes $[F(2.42)=2.05$, $\left.\eta p^{2}=0.09, p>.148\right]$.
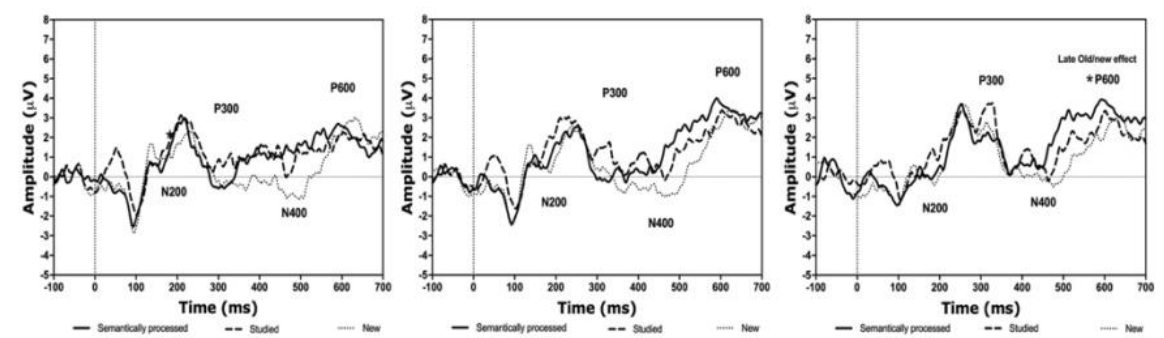

Figure 6. Grand averages across participants for the N200, P300, N400 and P600 during the recognition of the non-words according to stimulus type at the frontal (A), central (B), and posterior (C) electrode location clusters. Stimulus type effects: ${ }^{*}: p<.05$ 

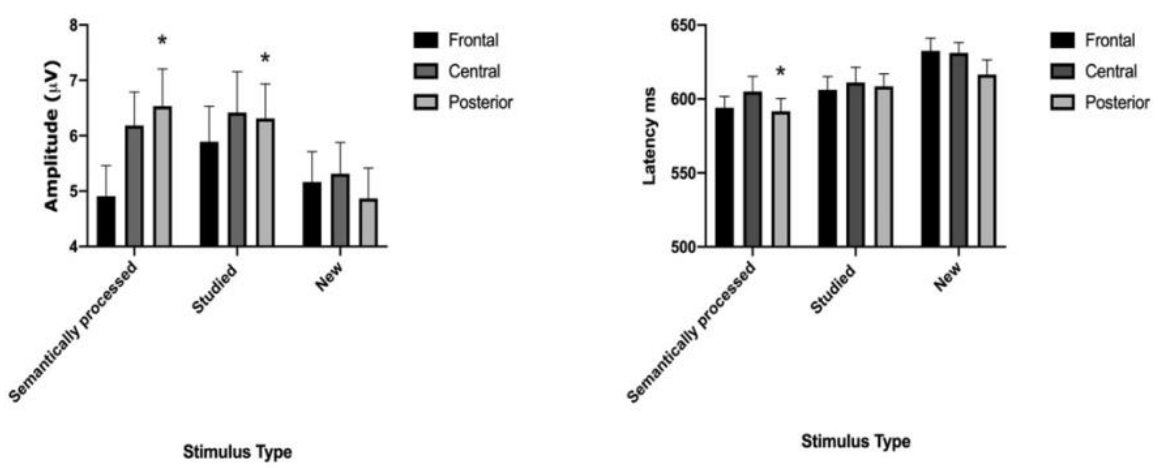

Figure 7. Means of the P600 amplitude (A) and latency (B) during the recognition of the nonwords. Stimulus type effects: different from the semantically processed items: ${ }^{*}: p<.001,{ }^{*}: p<$ .05 , different from the studied items: \#: $p<.05$

As for the latency, there was a significant main effect of stimulus type $[F(2.20)=3.97, \eta p 2=0.28, p<.035$; see Figures $6 \mathrm{~A}-\mathrm{C}$ and $7 \mathrm{~B}]$. Post-hoc tests showed that the P600 latency related to the strong items was shorter compared to the new $(p<.027)$. No other differences were found (all associated $p$-values $>$ .482). Finally, the interaction between stimulus type and location was not significant $[F(4.84)=0.67, \eta p 2=0.03, p>.546]$.

\section{III.5. Discussion}

In the current study, we examined the role of memory strength in the old/new discrimination process involving abstract figures and non-words. To account for the effect of memory strength, we manipulated LOP and used repetition. The present findings, using pre-experimentally unfamiliar and meaningless abstract figures and non-words, are in line with the notion that discrimination performance depends on how strong a particular newly formed memory is. Namely, correct identification of the new abstract figures and nonwords was superior to the recognition of the old items when they were merely 
studied, and thus, relied on weaker memories (shallowly memorized and not repeated). This was not the case when the stimuli were drawn or semantically processed, and thus, relied on stronger memories (deeply memorized and repeated). Additionally, despite the unequal proportion of old and new items (2:1) during the recognition phase, we did not detect an 'old' response bias for either the abstract figures or the non-words.

The EEG analysis indicated that the amplitude of the N200 was more negative during the processing of the studied than the drawn figures at the posterior location. The N400 was more negative for the new than the drawn and the studied figures at the fronto-central FN400 (early old/new effect) and at the posterior location. In addition, the drawn figures elicited a larger P600 amplitude in comparison to the studied and new stimuli at the centro-parietal locations (late old/new effect). Furthermore, the latency related to the processing of the new figures was shorter than that related to the studied. The ERPs of the non-words were not as clear and straightforward as seen with the abstract figures. The semantically processed non-words elicited larger P600 amplitudes than the studied or the new ones, representing a late old/new effect. There was also a large variety in the latencies. The only significant latency effect was detected for the P600. Namely, the latency associated with the processing of the semantically processed non-words was longer than that of the new. Below, we discuss how these ERP findings can explain discrimination performance.

\section{III.5.1. Abstract Figures}

As expected, we found that stronger memories achieved by deeper mnemonic LOP and repetition improved recognition accuracy and decreased the RT-s of the drawn (deeply memorized and repeated) as opposed to the studied (shallowly memorized) figures. These findings align with the notion that deeper LOP improves recognition and discrimination performance (Hulstijn, 1997; 
Paivio \& Desrochers, 1981; Solso, 1995). The current data also show that it was likely to be more difficult to correctly discriminate the studied abstract figures as being old or new since their discriminability indexes $\left(\mathrm{A}^{\prime}\right)$ were lower than those of the drawn. This result is consistent with previous research (Hirshman, 1995; Ratcliff, Clark, \& Shiffrin, 1990).

Furthermore, correct new item identification was superior when abstract image memory was weak (studied items memorized shallowly) but not when the memory was strong (drawn abstract figures that were deeply memorized and repeated). This differential effect of deep vs. shallow LOP and repetition vs. no repetition on the recognition performance indicates a mediating effect of memory strength. Prior research on discrimination performance involving well-known stimuli has typically shown that new items are processed more accurately than old ones (Donaldson, 1992; Kafkas \& Montaldi, 2014; Kafkas \& Montaldi, 2018; Kumaran \& Maguire, 2007). However, when new abstract memories are formed, it seems that familiar (old) abstract figurative items that rely on strong are processed more accurately than the unfamiliar (new) ones. Moreover, in line with previous findings, the drawn figures having the strongest memory were judged 'old' correctly the fastest (Delhaye, Bastin, Moulin, Besson, \& Barbeau, 2017; Kafkas \& Montaldi, 2014; Kafkas \& Montaldi, 2018).

When looking at the brain activity data, we found that the studied figures' processing elicited a more negative N200 amplitude than the drawn ones at the posterior location. In oddball tasks, the N200 amplitude increases in response to an unpredictable stimulus in a pool of frequently repeated items (Patel \& Azzam, 2005). The N200 has also been linked to a mismatch between an existing memory template and the test stimulus, thus perceiving something as new (Daffner et al., 2000). Moreover, this ERP is assumed to be sensitive to perceptual novelty in that a single repetition attenuates its amplitude (Ferrari, Bradley, Codispoti, \& Lang, 2010). Thus, on the one hand, it could be that the weakly embedded abstract figures were regarded as relatively new, which 
explains why their N200 amplitudes increased. On the other hand, the drawn figures were repeated, which could account for their attenuated amplitudes.

Furthermore, in agreement with Beisteiner et al. (1996), and in contrast to O'Hare and Goodwin (2018), we failed to find differences for the P300. This can be explained by the nature of the images and experimental designs used in these experiments. Namely, we used simple black and white abstract line drawings in an old/new recognition memory paradigm. Likewise, Beisteiner et al. (1996) used black and white abstract geometric images and tested old/new recognition. O'Hare and Goodwin (2018) used rather complex and colorful abstract artwork and a viewing task accompanied with discomfort judgements. Thus, while brain responses might differ depending on the complexity of the abstract imagery, it seems that old/new discrimination of simple abstract figures neither affects the P300 amplitude nor the latency.

However, the current data showed a greater negative deflection of the N400 for the new compared to the drawn, and the studied compared to the drawn abstract figures over the fronto-central and posterior regions. This finding supports the notion that the N400 reflects familiarity-based discrimination and correct identification of new or fragilely memorized items (Curran \& Hancock, 2007; Rugg \& Curran, 2007). The more posterior N400 is typically related to semantic processing (Otten, Sveen, \& Quayle, 2007; Ziegler, Besson, Jacobs, \& Nazir, 1995). Thus, the finding that the drawn abstract figures evoked the largest amplitudes at the more posterior locations could indicate that the participants tried to use verbalization during the processing of these stimuli. However, we did not ask which memory strategies the participants used.

The P600 amplitudes of the drawn figures were larger compared to the new and studied ones. Since this ERP component is known to reflect recollection memory and successful old/new discrimination (Curran \& Hancock, 2007; Friedman \& Johnson, 2000; Otten, Sveen, \& Quayle, 2007; Rugg \& Curran, 2007; Ziegler, Besson, Jacobs, \& Nazir, 1995), this could indicate that the drawn figures 
and their encoding context were recollected better, probably as a function of the memory strength manipulation. Our behavioral results also supported such a notion.

To summarize, the superior discrimination performance for the new figures can be explained by a more negative N400 amplitude. Larger P600 peaks can explain the improved recognition of the deeply memorized and repeated (drawn) figures. The N400 amplitude related to the processing of the studied figures was comparable to the new ones and showed a P600 wave similar to them, which elucidates their poorer discrimination performance. Thus, accurate discrimination performance of the abstract figures can be explained by a combination of effective use of familiarity (N400) and recollection (P600) type memory for the deeply encoded and repeated items.

\section{III.5.2. Non-Words}

The behavioral findings regarding the advantages of deeper mnemonic encoding and repetition were very similar for the non-words and the abstract figures. Thus, semantically processed non-words were endorsed correctly as 'old' just as well as the new ones were identified as 'new'. Additionally, deeper LOP and repetition resulted in improved accuracy compared to shallow memorization without repetition. Our findings on the LOP effect with the non-words were comparable to those detected with actual words by Gardiner (1988). Half of the participants encoded well-known words in his experiment by writing down a rhyming word (intermediate encoding); the other half had to come up with a semantic associate for each word (deep encoding). After a delay, an old/new recognition test was applied. Accuracy scores in the deep condition were significantly higher than in the intermediate condition. Thus, it can be said that deeper compared to more shallow LOP does not only improve the discrimination of words, but also of non-words. 
The reaction times in response to the new non-words were slower than to the studied. Similar findings have previously been reported with non-words (Fjell, Walhovd, \& Reinvang, 2005), whereas the opposite was found with words (Van Petten \& Senkfor, 1995). It could be that the nonsense letter combinations of the new non-words in the current experiment were simply more challenging to process due to some sort of confusion or conflict with existing verbal knowledge, which led to more extended processing. In other words, it might be difficult for the brain to make sense of random letter strings upon a first encounter.

Although the behavioral results were similar to the abstract figures, EEG processing appeared to be different due to the diverse nature of the stimuli. Notably, the ERP components visible for the non-words appeared to be less clear than those of the abstract figures or components typically observed with words (Smith \& Guster, 1993; Van Petten \& Senkfor, 1995). Similar visual data can be found in the experiment by Otten, Sveen, and Quayle (2007), who investigated the electrophysiological processing of words and non-words during encoding and recognition. The most feasible explanation for the qualitatively worse representation of the word vs. non-word ERPs could be that only the former has meaning. This enables us to obtain clearer signals during word over non-word processing since actual words make sense.

In contrast to our expectations, we failed to detect significant N200 and P300 effects or find support for the context updating hypothesis with the nonwords (Patel \& Azzam, 2005). We also could not detect differences involving the N400 amplitude or latency. This can be explained by the non-words being semantically incorrect (Ziegler, Besson, Jacobs, \& Nazir, 1995). Indeed, other studies using meaningless stimuli also did not find effects on the N400 (MacKenzie \& Donaldson, 2009; Voss \& Paller, 2009). However, this contrasts with our own findings regarding the abstract figures, which were also meaningless. 
As seen with the abstract figures, the P600 amplitude of the non-words was also different for the semantically processed, studied, and new stimuli. Namely, while the semantically processed and the studied non-words elicited similar ERPs, they were both larger than those of the new. Such late old/new effects align with previous research showing that the later posterior P600 reflects recognition memory and effective old/new discrimination (Curran \& Hancock, 2007; Friedman \& Johnson, 2000; Otten, Sveen, \& Quayle, 2007; Rugg \& Curran, 2007; Ziegler, Besson, Jacobs, \& Nazir, 1995). As such, our results agreed with those by Fjell, Walhovd, and Reinvang (2005), who used both word and nonword stimuli in a two-phase EEG memory experiment. As in the current paradigm, their first phase was meant to induce deeper LOP utilizing a lexical discrimination task (word or non-word), followed by an old/new recognition test. Their finding revealed a clear parietal P600 peak for both the old words and the non-words, which the authors attributed to the quality and quantity of the recollected episodic memory. Thus, it seems that the deep memorization task applied with the semantically processed non-words, similar to the drawn abstract figures, contributed to memory recollection. This notion aligns with our finding, according to which the P600 latency associated with the new non-words was shorter than that related to the semantically processed ones. Accordingly, processing of the semantically processed items might have been more demanding due to recollection of contextual details (pairs with the rhyme words during deep memorization). Our findings also indicate that memory formation and retrieval of the semantically processed and the studied items were likely successful in the brain. Nonetheless, the recognition accuracy of the studied stimuli was worse than that of the semantically processed and new non-words. 


\section{III.6. Conclusions}

The present study shows that the discrimination performance of preexperimentally unfamiliar abstract figures and non-words depends on memory strength induced by deeper LOP and repetition. Specifically, new abstract figures and non-words were more accurately identified than the old ones that relied on weak memories. In contrast to the stimuli relying on weak memories, discrimination of the drawn/semantically processes and new stimuli was improved when their memory was strong or when the memory was absent, respectively. By using pre-experimentally unfamiliar abstract figures and nonword stimuli, the current experiment largely controlled for retroactive interference.

The EEG results of the abstract figures indicated that the N400 effect discriminates between the new and drawn items relying on strong memories, whereas the P600 effect discriminates between the recollected drawn and nonrecollected items (studied items with weak memory and new stimuli). The late parietal P600 likely differentiates between old and new non-words.

In closing, the presented findings fill a gap in the research by providing new insight into how the brain processes and discriminates abstract figures and nonwords and what role memory strength has in these processes. Essentially, our findings improve the understanding of such visual and lexical memory processing that does not rely on semantic knowledge. Moreover, we could identify a pattern of brain activity that defines accurate discrimination performance of such items, which could be useful in developing appropriate BCI technologies.

To further investigate the claims presented in this work, future research could implement spectral power analyses. Moreover, the present paradigm could be extended with the application of confidence judgments and questionnaires that could reveal which specific strategies the participants used during 
memorization. An intriguing follow-up study could examine the relation between recognition and recall using an extended version of the current paradigm. Finally, it would be interesting to define the electrophysiological patterns of pre-experimentally unfamiliar old/new item recognition and combine these results with BCI technology. 
IV. Chapter:

Age Effects on old/new Recognition

Performance involving Abstract Figures

and Non-Words

Monika Toth, Anke Sambeth \& Arjan Blokland

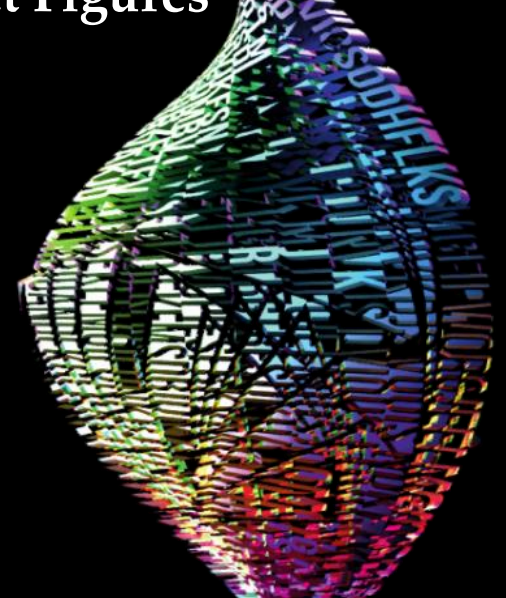

Submitted for publication

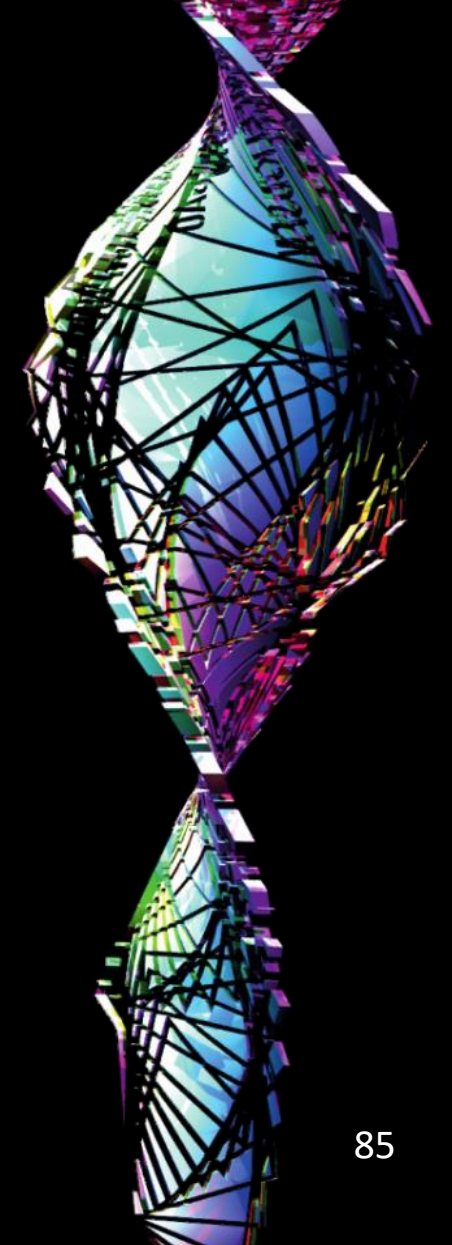




\section{IV.1. Abstract}

Background: Age-related memory problems posit a growing concern in our society. This study investigated the impact of age and memory strength on the recognition performance of pre-experimentally unfamiliar abstract figures and non-words.

Methods: We applied a three-phase old/new recognition memory paradigm and manipulated memory strength as a function of the Levels of Processing (deep vs. shallow) and repetition.

Results: Older adults relative to the young showed impairment in the correct identification of new items. As indicated by the lower discriminability indexes, the elderly also had difficulties discriminating the strongly (drawn) and the weakly (studied) embedded abstract figures but not the non-words. Age-related differences in speed of processing were also only evident with the abstract figures. Additionally, our results revealed that discrimination performance was mediated by memory strength in both age groups.

Discussion: The current findings agree with previous research on age-related impairment in new item recognition, which can be attributed to misrecollection, decreased sensitivity to novelty, and less accurate novelty assessment in the elderly than the young. The detected age effects on the discriminability of the drawn and studied abstract figures agree with the age-related impairment in the perceptual encoding hypothesis and support the notion related to the need for environmental support to reduce age effects.

Conclusion: The present findings delineate differential age effects on the memory processing of visual and verbal materials. Thus, the current study further supports the notion that recognition performance in aging is only impaired under certain conditions. Finally, the current results extend the understanding of the mechanisms underlying age-related changes on the memory of pre-experimentally unfamiliar items. 
Keywords: discrimination; recognition memory; cognitive aging; abstract figures; non-words; Levels of Processing; memory strength 


\section{IV.2. Introduction}

Age-related memory problems are among the most prominent complaints of the elderly (Fraundorf, Hourihan, Peters, \& Benjamin, 2019). For example, it has been shown that older participants compared to young have difficulties with learning new information, especially when the study process requires self-initiated strategies (Bridger et al., 2017; Fraundorf, Hourihan, Peters, \& Benjamin, 2019; Naveh-Benjamin \& Mayr, 2018). Moreover, based on oddball tasks, older people have been found to demonstrate decreased sensitivity to novelty (Fandakova, Lindenberger, \& Shing, 2014), likely resulting from the inability to inhibit irrelevant information (Amenedo \& Diaz, 1998; Hasher, Zacks, \& May, 1999; Weisz \& Czigler, 2006). Also, retrieval processes, such as free recall and recognition are assumed to show age deficits (Bridger et al., 2017; Fraundorf, Hourihan, Peters, \& Benjamin, 2019; Naveh-Benjamin \& Mayr, 2018), which has partly been attributed to set back in processing speed (Levin, Eisenberg, \& Benton, 1992; Salthouse, 1996; van Hooren et al., 2007). Although age-related memory deficits in tasks requiring novelty detection, recall, and memory for context are well understood, differences in the processes underlying recognition memory remain unclear.

Information-processing theories define recognition as a predicament of discrimination between what is known (familiar) and what is not known (new) (Benjamin, Diaz, \& Wee, 2009; Stanislaw \& Todorow, 1999; Verde \& Rotello, 2007; Yonelinas, 2002). Discrimination performance is typically tested with paradigms that assess recognition memory. In such paradigms, participants must recognize previously studied items as old correctly and identify previously not seen ones as new (Malmberg, 2008). The recognition/discrimination process generates a cue, which can prompt either a sense of familiarity or novelty (Yonelinas, 2002; Yonelinas, Kroll, Dobbins, Lazzara, \& Knight, 1998). Thus, the decision process determines whether a stimulus is judged as old or as new. In this context, 
participants rely on the fidelity of their memory and their preference when making the old/new decisions.

A recent meta-analysis by Fraundorf, Hourihan, Peters, and Benjamin (2019) examined age effects on recognition. They found that older participants exhibit poorer recognition accuracy, use more liberal decision criterions, and are more biased towards judging an item 'old' even when it is new. For example, Norman and Schacter (1997) found that the elderly were more susceptible than the young to falsely remember information that was not presented during encoding but was semantically related to the studied words. Such findings could indicate a global memory deficit, which entails an overall memory performance decrease rather than an impairment of a specific memory process (Benjamin, 2010). In contrast, another review by Park and Festini (2017) argues for domainspecific memory deficits and points out that age differences are more evident with complex than easy tasks. This notion aligns with the limited resources theory of cognitive aging (Craik \& Byrd, 1982; Park \& Festini, 2017). According to this hypothesis, age effects on memory are due to the elderly having limited amounts of cognitive resources (i.e., attention or mental energy) available during memory processing, which explains why task difficulty might lead to the observed age-effects.

Research has shown that age effects can be reduced, for instance, when cues are given during test (i.e., environmental support hypothesis) (Craik, 1986) or when the elderly are encouraged to engage in higher-level encoding (i.e., production deficit hypothesis) (Kausler, 1970), resulting in deeper Levels of Processing and stronger memory formation (Craik, 2002; Craik \& Rose, 2012). Previous studies have shown that memory strength can effectively be manipulated as a function of repetition (Verde \& Rotello, 2007) or depth of processing, the so-called Levels of Processing (LOP) (Gardiner, 1988). Repetition is known to strengthen memory by increasing the subjective sense of familiarity resulting from the re-encoding of a particular memory trace (Hintzman \& 
Curran, 1997; Ranganath \& Rainer, 2003). The LOP theory predicts that deep (e.g., meaning-extraction, pattern recognition, activation of prior knowledge) and intermediate processing (e.g., phonetics) lead to superior and faster retrieval when compared to shallow processing (e.g., perceptual analyses, rehearsal) (Craik, 2002; Craik \& Lockhart, 1972; Craik \& Tulving, 1975; Newell \& Andrews, 2004).

In agreement with the above, the processing theory account of cognitive aging suggests that the memory problems of the elderly are due to difficulties with commencing mnemonic processing when no experimental instructions are provided and can be improved when such instructions are given (Craik, 2002). Mnemonics, such as verbal (e.g., rhyming), motor (e.g., drawing), or visual (e.g., imagining) techniques, strengthen memory storage and improve subsequent retrieval due to deeper elaborative processing (Hulstijn, 1997; Jones-Gotman, 1986; Paivio \& Desrochers, 1981; Solso, 1995). Mnemonics are useful because they involve the allocation of several cognitive domains (Hulstijn, 1997; Paivio \& Desrochers, 1981; Solso, 1995) from which the aged can particularly benefit (Fraundorf, Hourihan, Peters, \& Benjamin, 2019).

However, the pictures and words used in most experiments are rarely wholly novel in that they would not have an existing representation in memory (Fraundorf, Hourihan, Peters, \& Benjamin, 2019). As such, discrimination of such stimuli relies pre-dominantly on pre-experimental knowledge, which, especially in older people, entails a lifespan of experiences with similar items. Therefore, it is less surprising that the aged exhibit a deficit since their more extensive experiences might be a source of confusion as to whether a stimulus was memorized during or beforehand a study. Also, aged adults tend to use their existing semantic knowledge more efficiently, which, in fact, depending on the stimuli used, can sometimes improve their discrimination performance (Badham, Poirier, Gandhi, Hadjivassiliou, \& Maylor, 2016; de Chastelaine, Mattson, Wang, Donley, \& Rugg, 2017; Fraundorf, Hourihan, Peters, \& Benjamin, 2019). 
Pre-experimentally unfamiliar stimuli, such as abstract figures or nonwords, hardly rely on existing semantic knowledge, and therefore, can adequately control for confusion due to prior experience. For example, Smith, Park, Cherry, and Berkovsky (1990), similarly to Harker and Riege (1985), reported significant age differences during the recognition of meaningless abstract but not meaningful, concrete pictures. Another study by Badham and Maylor (2011) compared the discrimination performance of words and nonwords in healthy elderly and young participants using an associative recognition memory paradigm. Age differences in the discrimination of the non-words were comparable to the words, notwithstanding a lack of pre-experimentally existing memory for non-words.

In the present study, we aimed to examine age effects on the recognition performance as a function of memory strength involving meaningless abstract figures and non-words (Toth, Sambeth, \& Blokland, 2021). We chose to include both figurative and verbal stimuli since, on the one hand, processing of such stimuli can show differential age effects (Badham \& Maylor, 2011; Koutstaal, Reddy, Jackson, Prince, Cendan, \& Schacter, 2003). On the other hand, these stimuli are also known to be processed differently (Kim, Yoon, \& Park, 2004). To investigate this, we tested aged and young participants with a three-phase old/new memory paradigm. The stimuli were first familiarized via mnemonic encoding to prompt deep processing: the participants had to redraw the abstract figures and mention existing rhyming words for the non-words (semantic processing). Then, the stimuli in the second phase were processed shallowly via an instruction to remember as many stimuli as possible. Here, the stimuli from the first phase were shown repeatedly mixed with some new items. Finally, in the third phase, an old/new recognition test was used. Here, stimuli from the first and second phases were shown together with new items. Recognition accuracy and speed were assessed. 
Based on the presented evidence, we hypothesized that the overall recognition performance would show age effects. Specifically, we expected to see larger differences for to the studied and new items likely resulting from higher false alarm rates in the aged then the young group. In addition, no age effects were anticipated concerning the recognition of the deeply memorized items. Also, the older participants were expected to be slower in processing than the young. Overall, the correct identification of the new items as opposed to the studied was expected to be better (Toth, Sambeth, \& Blokland, 2021). Finally, recognition of the deeply vs. shallowly encoded items was expected to be more accurate independently of age.

\section{IV.3. Materials and Methods}

The Ethical Committee of the Faculty of Psychology and Neuroscience of Maastricht University granted ethical approval for this experiment. Each participant received monetary compensation or research participation credit points. On average, the experiment took 1.5 hour/test-session (Ethical Approval Code: ECP13_02_2012).

\section{IV.3.1. Participants}

An a priori statistical power analysis using $G^{*}$ power 3.1 showed that in order to detect significant effects using an ANOVA 15 participants were required for each age group, with an effects size of 0.4 and power of at least $80 \%$ at a significance level of 5\% (Faul, Erdfelder, Lang, \& Buchner, 2007). The main inclusion criteria were age (young: 18-30 years, aged: 60-80 years) and being fluent in the Dutch language. A total of 15 young healthy participants (5 males) with mean age of 23 years, and 15 healthy aged participants (8 males) with a mean age of 71 years were recruited by means of advertising. The aged participants 
were excluded if suffering from any objectified memory impairment or neurological/neuropsychiatric disorder. In order to exclude severe cognitive impairment, the Mini Mental State Examination (MMSE) was administered, and volunteers who scored $\geq 25$ indicating dementia, were excluded (Folstein, Folstein, \& McHugh, 1975).

\section{IV.3.2. Procedure}

The procedure and the stimuli were the same as applied in another study by our group (Toth, Sambeth, \& Blokland, 2021). Thus, after signing informed consent, participants were admitted to the study. Prior to starting the experiment, each participant filled in a demographic questionnaire, including information about sex, age, and handedness. During the test, stimuli were presented via a computer screen, and participants had to respond on two keys of a response pad. Recognition accuracies and reaction times were recorded.

A memory paradigm with abstract figures and non-words was applied in separate tests. See Figure 1 for an example of the stimuli used. Every participant performed each test phase first with the abstract figures and then with the non-words to minimize verbalization of the figurative stimuli. The experiment consisted of three phases (see Figure 2). In phase 1 (deep memorization leading to 'strong' memory), participants were familiarized with a series of 30 abstract figures or monosyllabic non-words in separate tests (list 1: L1). Participants were asked to manually redraw the abstract figures on an answer sheet in order to induce deep LOP. They had to mention existing English or Dutch rhyme words for each non-word to induce intermediate LOP. Stimuli were presented for $1 \mathrm{~s}$, and the participants were given $14 \mathrm{~s}$ to execute the deep encoding task. If they were ready earlier, they could press a button, and $2 \mathrm{~s}$ later, the next stimulus appeared. Stimuli were extracted from previous studies 
(Glosser, Friedman, Grugan, Lee, \& Grossman, 1998; Redoblado, Grayson, \& Miller, 2003; Seidenberg, Plaut, Petersen, McClelland, \& McRae, 1994).

\begin{tabular}{|l|l|l|l|}
\hline \multicolumn{3}{|c|}{ Abstract Figures } & \multicolumn{3}{c|}{ Non-Words } \\
\hline & & & \\
\hline
\end{tabular}

Figure 1: Examples of the stimuli used.

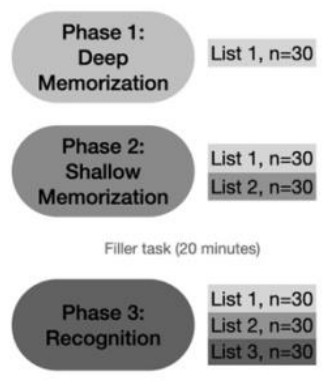

Figure 2. Schematic overview of the experimental design. Phase 1: deep memorization with the preexperimentally unfamiliar abstract figures and non-words in separate tests using a mnemonic encoding task (redrawing the abstract figures and mentioning rhyming words for the non-words). The 30 stimuli used here form List 1 (drawn/semantically processed stimuli). Phase 2: shallow memorization with the instruction to remember as many stimuli as possible. This phase contained items from List 1 and 30 new ones (List 2, studied stimuli). Phase 3: recognition of the stimuli including List 1, List 2, and 30 new (List 3).n: number of stimuli presented (Toth, Sambeth, \& Blokland, 2021). 
During phase 2 (shallow memorization leading to 'weak' memory), participants were instructed to remember as many stimuli as possible. In this phase, 60 stimuli (abstract figures or non-words) were used: 30 stimuli from L1 were randomly mixed with 30 new ones (L2). All stimuli were shown for $1 \mathrm{~s}$ with an inter-stimulus interval (ISI) of $2 \mathrm{~s}$.

During phase 3, participants were asked to decide if they had seen the presented stimulus in the previous series (L1 and L2) or whether the stimulus was new to them (L3: new, $n=30$ ). The 90 non-words or abstract figures were presented for a duration of $1 \mathrm{~s}$, or less in the case of faster button press; the ISI was $2.5 \mathrm{~s}$. Participants had to press the corresponding buttons ('old' for L1 and L2, or 'new' for L3 stimuli) on a response box as quickly and accurately as possible.

A filler paper-and-pencil task and another non-verbal task were given between phase 2 and 3 . The filler task consisted of the localization of number sequences, vertically or horizontally placed within a field of numbers (10 min). The other task consisted of watching a silent cartoon while auditory stimuli were presented (10 $\mathrm{min})$.

\section{IV.3.3. Data Analysis}

As reported in Toth, Sambeth, and Blokland (2021), before analysis, all data were evaluated for having normal distribution and homogeneity of variance. Additionally, raw data were checked for outliers. Outlier values were replaced with their regression estimates produced by the missing value analyses (IBM SPSS Statistics for Macintosh, Version 27.0. Armonk, NY: IBM Corporation). Additionally, due to technical issues, 1-2 responses per participant were missing (e.g., the button press was not recorded). In these cases, values were replaced with their regression estimates. Effect sizes are reported based on partial eta- 
squared ( $\eta p 2)$ data. Furthermore, Mauchly's test of sphericity was applied. In case the assumption of sphericity was violated, a Greenhouse-Geisser correction was used. In all cases, degrees of freedom of assumed sphericity were reported. Levene's test was used to assess the equality of variances. In case the assumption of equal variances was violated, median-based independent samples nonparametric tests were applied. Post-hoc comparisons and simple effects were investigated using paired-samples and independents samples t-tests, applying adjustments for multiple comparisons; the observed p-values were multiplied by the number of comparisons, which was tested against the set significance level of 0.05. Values of unequal variances are reported if the assumption of equal variances was violated.

For the behavioral data, Signal Detection Theory (SDT) was applied in order to investigate the discrimination performance (Benjamin \& Bawa, 2004; Benjamin, Diaz, \& Wee, 2009; Stanislaw \& Todorow, 1999; Verde \& Rotello, 2007). Discrimination accuracy was defined as the ability to distinguish the different types of stimuli (drawn/semantically processed, studied and new). Correct responses included an 'old' response to the drawn/semantically processed items and the studied stimuli, and a 'new' response to the new items. Incorrect responses involved a 'new' response to the drawn/semantically processed items and the studied stimuli and an 'old' response to the new stimuli. See Table 1 for an overview. 
Table 1. Overview of the different types of responses as a function of stimulus type.

\begin{tabular}{ccc}
\hline & Stimulus Type & Response \\
\hline Hit $(\mathrm{H})$ & $\begin{array}{c}\text { Drawn or semantically } \\
\text { processed/Studied }\end{array}$ & 'Old' \\
\hline Miss (M) & $\begin{array}{c}\text { Drawn or semantically } \\
\text { processed/Studied }\end{array}$ & 'New' \\
\hline Correct Rejection (CR) & New & 'New' \\
\hline False Alarm (FA) & New & H/(H + M) \\
\hline Hit Rate (HR) & Drawn or semantically \\
processed/Studied & $\mathrm{CR} /(\mathrm{CR}+\mathrm{FA})$ \\
\hline
\end{tabular}

Given the memory strength manipulation in the current design (deep memorization, shallow memorization, and recognition), the correct response rates, being hit rates (HR) for the drawn/semantically processed, and the studied items and correct rejection rates (CRR) for the new, were used to evaluate the discrimination accuracy. Furthermore, to investigate discriminability, nonparametric $\mathrm{A}^{\prime}$ statistics were computed for the drawn/semantically processed and the studied stimuli using Equations (1) or (2) (see below). $\mathrm{A}^{\prime}$ varies from 0 to 1 , with 0.5 indicating chance performance. Higher values are indicative of improved performance (Snodgrass \& Corwin, 1988; Stanislaw \& Todorow, 1999).

$$
\begin{aligned}
& \mathrm{A}^{\prime}=0.5+\frac{(H R-F A R)(1+H R-F A R)}{4 H R(1-F A R)}, \text { if } H R \geq F A R \\
& \mathrm{~A}^{\prime}=0.5-\frac{(H R-F A R)(1+H R-F A R)}{4 H R(1-F A R)}, \text { if } H R<F A R
\end{aligned}
$$

$\mathrm{A}^{\prime}$ : discriminability index, HR: hit rate, FAR: false alarm rate.

During recognition, the a priori probabilities of old and new items and the quality of the match between a test item and the memory for studied items can influence the bias parameter (Huang \& Ferreira, 2020; Stanislaw \& Todorow, 1999). Such a model does not fit the current paradigm due to the memory strength manipulation used and the equivalent proportion and intended comparison of the strong $(n=30)$, weak $(n=30)$, and new items $(n=30)$ [44]. After all, the final 
proportion of 'old' and 'new' responses was 2:1. Therefore, we calculated the total amount of 'old' (H+FA) and 'new' $(M+C R)$ responses given by the participants. This was done to examine whether there was a preference for either the 'old' or 'new' responses. Results were compared using paired samples t-tests with Bonferroni corrections.

RT data of the hits were evaluated, as well. To be able to use parametric tests, RT-s were transformed into $|\log (1 / \mathrm{RT})|$ to obtain a normal distribution of the data (Osborne, 2002). Moreover, the median RT data are reported as central tendency parameters, together with the corresponding first and third interquartile ranges (Ratcliff, 1993).

Statistical analysis was conducted using SPSS 27.0. A repeated measures analysis of variance (ANOVA) was used to investigate discrimination accuracy scores and RT-s for the different stimuli in the different categories as assessed in Phase 3. The within-subject variables were Stimulus Type (drawn/semantically processed, studied stimuli, and new) for the abstract figures and non-words analyzed in separate tests, and the between subject variable was Age (young and elderly).

\section{IV.4. Results}

Although there was an unequal number of old-responses over newresponses (2:1), we found that there was no response bias (see Table 2). The mean signal-detection parameter estimates are displayed in Table 3. 
Table 2. The overall number of old and new responses during the recognition phase Data represent the means (SEM) of the total 'old' and 'new' responses and the corresponding \% compared to the 90 items/stimulus category (abstract figures and non-words), and the t-statistics for the young and the elderly.

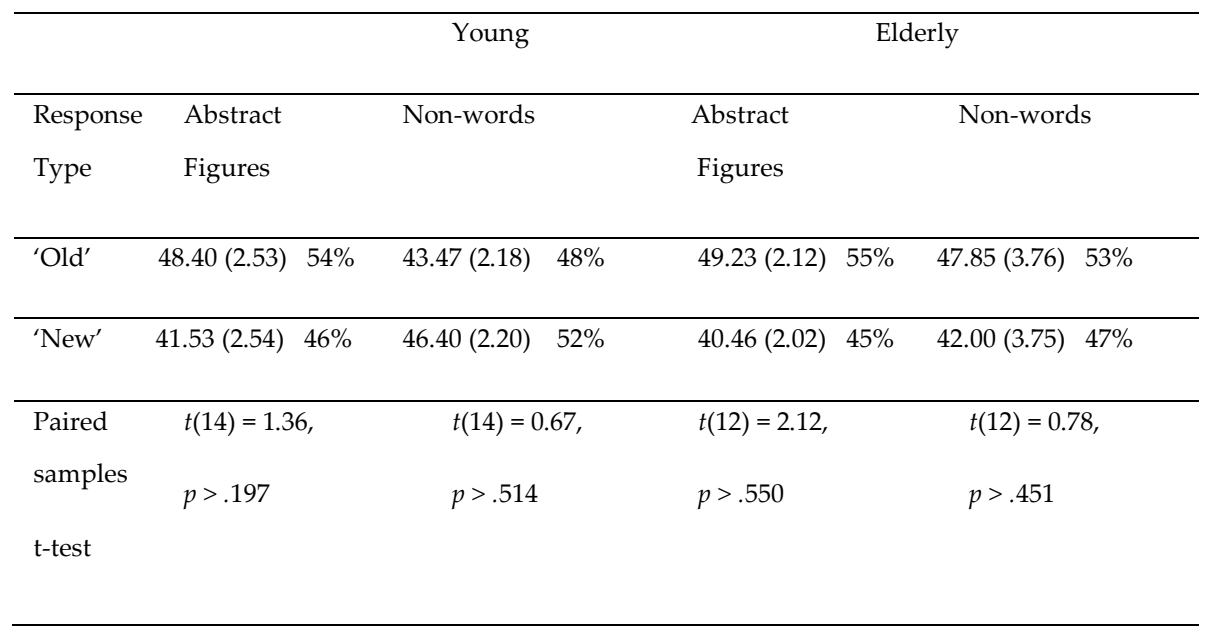

Table 3. Means (SEMs) of the signal-detection measures concerning the recognition performance with the abstract figures and non-words for the drawn/semantically processed, studied, and new stimuli according to age (young and elderly).

\begin{tabular}{|c|c|c|c|c|c|}
\hline \multirow[b]{2}{*}{ Stimulus } & \multirow[b]{2}{*}{ Parameters } & \multicolumn{2}{|c|}{ Young } & \multicolumn{2}{|c|}{ Elderly } \\
\hline & & Abstract & Non-words & Abstract & Non-words \\
\hline Type & & Figures & & Figures & \\
\hline \multirow{2}{*}{$\begin{array}{l}\text { Drawn/Se } \\
\text { mantically } \\
\text { processed }\end{array}$} & HR & $0.98(0.01)$ & $0.80(0.04)$ & $0.94(0.12)$ & $0.83(0.02)$ \\
\hline & $\mathrm{A}^{\prime}$ & $0.90(0.10)$ & $0.69(0.02)$ & $0.76(0.03)$ * & 0.62.(0.01) \\
\hline \multirow[t]{2}{*}{ Studied } & HR & $0.57(0.06)$ aa, bb & $0.50(0.04)^{a a, b b}$ & $0.52(0.05)^{a a, b b}$ & $0.57(0.05)$ aa, bb \\
\hline & $\mathrm{A}^{\prime}$ & $0.63(0.03)^{\text {aa }}$ & $0.56(0.01)^{\text {aa }}$ & $0.56(0.01)^{*}$, aa & $0.55(0.01)^{\text {aa }}$ \\
\hline New & CRR & $0.95(0.01)$ aa & $0.84(0.02)$ & $0.80(0.01)^{*}$, a & $0.70(0.02)$ * \\
\hline
\end{tabular}

HR: hit rate, CRR: correct rejection rate, $\mathrm{A}^{\prime}$ : discriminability index. Age effects: ${ }^{*}: p<.05$. Stimulus type effects: different from the drawn items: aa $p<.001$, different from the new items: $b b p<.001$. 


\section{IV.4.1. Abstract Figures}

When analyzing the accuracy performance in the session with the abstract figures the ANOVA revealed a significant age $x$ stimulus type interaction $\left[F(2,25)=4.00, \eta p^{2}=0.34, p<.031\right.$; see Fig. 3A and Table 3]. Levene's test indicated unequal variances for the new abstract figures $[F(1,26)=6.79, p<.015]$. Simple effects analyses revealed that the young compared to the elderly were more accurate in recognizing the new abstract figures $[t(12.38)=3.36, p<.015]$. No such differences were found for the drawn $[t(26)=2.52, p>.054]$ and the studied items $\left[t(26)=0.63, p>\right.$.999]. Also, the main effect of age $\left[F(1,26)=6.13, \eta p^{2}=0.19, p<\right.$ $.020]$ and stimulus type were significant $\left[F(2,52)=76.67, \eta p^{2}=0.75, p<.001\right.$; see Fig. 3A and Table 3]. Post-hoc tests of the stimulus type showed that overall recognition accuracy of the drawn stimuli was better compared to the studied ( $p$ $<.001)$ and new $(p<.001)$. Also, more new stimuli were endorsed correctly compared to the studied $(p<.001)$.

Figure 3: Recognition accuracy performance of the abstract figures (A) and the non-words (B) according to age (young and elderly) for the hit rates of the drawn/semantically processed and studied items, and the correct rejection rates in repose to the new items. The bars represent the means.

A

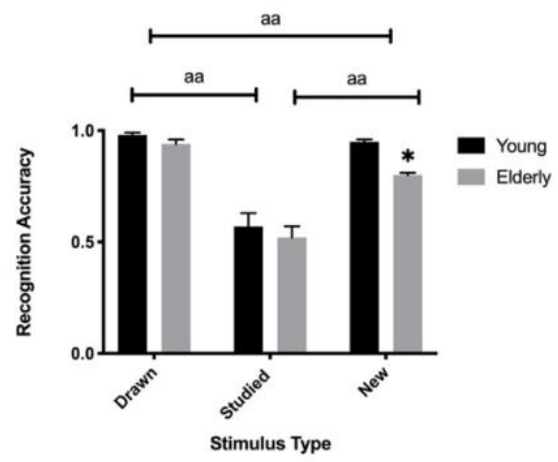

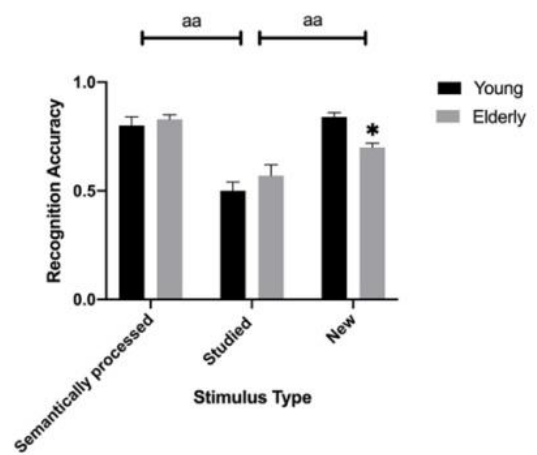

Age effects: ${ }^{*}: p<.05$. Stimulus type effects: aa: $p<.001$ 
The analyses performed on the $\mathrm{A}^{\prime}$ scores revealed a significant age $\mathrm{x}$ stimulus type interaction $\left[F(1,26)=4.84, \eta p^{2}=0.16, p<.037\right.$; see Table 3]. Levene's test indicated unequal variances for the drawn $[F(1,26)=5.83, p<.023]$ and the studied abstract figures $[F(1,26)=4.71, p<.039]$. Simple effects analyses revealed that the young compared to the elderly could discriminate the drawn $[t(14.82)=$ $4.77, p<.001]$ and the studied abstract figures better $[t(22.22)=2.40, p<.025]$. As for the stimulus type effect, the drawn abstract figures resulted in improved discriminability compared to the studied $\left[F(1.26)=203.47, \eta p^{2}=0.89, p<.001\right.$; see Table 3]. The main effect of age was also significant $\left[F(1.26)=203.47, \eta p^{2}=0.89, p\right.$ $<.001]$.

When analyzing the RT-s, the ANOVA confirmed a significant main effect of age $\left[F(1,27)=19.56, \eta p^{2}=0.43, p<.001\right.$; see Table 3], with the young participants being faster than the elderly. Another main effect of stimulus type was found $\left[F(2,25)=85.29, \eta p^{2}=0.87, p<.001\right.$; see Table 3]. Post-hoc tests showed that reactions were faster to the drawn compared to the studied and compared to the new stimuli $(p<.001)$ in both age groups. No such difference was found between the studied and new stimuli $(p>$.999). Finally, there was no interaction between age and stimulus type $\left[F(2,52)=1.20, \eta p^{2}=0.09, p>.319\right]$. 
Table 4. Median reaction times (middle 50\% range; in milliseconds in response to the drawn/semantically processed, studied, and new abstract figures and non-words) and their corresponding first and third interquartile ranges for the young and the elderly.

\begin{tabular}{lcccc}
\hline \multicolumn{2}{c}{ Young } & \multicolumn{2}{c}{ Elderly } \\
\hline Stimulus Type & Abstract Figures & Non-words & Abstract Figures & Non-words \\
\hline Drawn/Semantically & 661 & 648 & $911^{* *}$ & 659 \\
processed & $(613-742)$ & $(636-665)$ & $(781-1029)$ & $(627-790)$ \\
\hline Studied & $794^{\text {aa }}$ & 650 & $1099^{* *}, a a$ & 620 \\
& $(718-1011)$ & $(575-719)$ & $(938-1202)$ & $(578-651)$ \\
\hline New & $811^{\text {aa }}$ & 689 & $1149^{* *}, a a$ & $684^{* *}$ \\
& $(734-953)$ & $(637-734)$ & $(1034-1210)$ & $(495-782)$ \\
\hline
\end{tabular}

Age effects: ${ }^{* *} p<.001$. Stimulus type effects: different from the drawn/semantically processed: $a a p<$ .001

\section{IV.4.2. Non-Words}

The ANOVA analysis of the non-words revealed a significant age $x$ stimulus type interaction $\left[F(2,25)=4.57, \eta p^{2}=0.27, p<.020\right.$; see Fig. $3 \mathrm{~B}$ and Table 3]. Levene's test indicated unequal variances for the semantically processed nonwords $[F(1,26)=6.79, p<.015]$. Simple effects analyses showed that the young compared to the older participants were more accurate in rejecting the new nonwords correctly $[t(26)=4.42, p<.003]$. No such differences were found for the semantically processed $[t(23.21)=0.66, p>.999]$ and studied items $[t(26)=1.54, p$ $>$.936]. Moreover, the main effect of stimulus type was also statistically meaningful $\left[F(2,25)=47.83, \eta p^{2}=0.79, p<.001\right.$; see Fig. 3B and Table 3]. Post-hoc tests showed that the recognition accuracy of the semantically processed stimuli was better compared to the studied $(p<.001)$ but not compared to the new items $(p>$.340). Also, more new non-words were identified correctly compared to the 
studied $(p<.001)$. Age did not yield a significant main effect $\left[F(1,26)=0.20, \eta p^{2}=\right.$ $0.01, p>$.659].

The analyses of the $\mathrm{A}^{\prime}$ scores revealed a significant age $\mathrm{x}$ stimulus type interaction $\left[F(1,26)=4.89, \eta p^{2}=0.16, p<.036\right.$; see Table 3]. However, simple effects analyses did not reveal any differences for the semantically processed $[t(26)=$ $4.77, p>.060]$ or the studied non-words $[t(26)=4.77, p>.060]$. As for the stimulus type effect, the semantically processed non-words resulted in improved discriminability compared to the studied $\left[F(1.26)=75.76, \eta p^{2}=0.75, p<.001\right.$; see Table 3]. The main effect of age was not significant $\left[F(1.26)=203.47, \eta p^{2}=0.89, p\right.$ $>$.070].

The analyses of the RT-s did not yield an age $\left[F(1,26)=0.62, \eta p^{2}=0.02, p\right.$ $>$.440], or stimulus type effect $\left[F(2,25)=1.98, \eta p^{2}=0.14, p>.159\right]$. Finally, the stimulus type $\mathrm{x}$ age interaction was statistically also not meaningful $[F(2,25)=$ $\left.0.26, \eta p^{2}=0.02, p>.773\right]$. See Table 4 .

\section{IV.5. Discussion}

The current study investigated age effects on the recognition performance of abstract figures and non-words using a three-phase old/new memory paradigm. We were particularly interested to see how aging affects the recognition of previously seen old vs. previously not seen new items when memory strength is manipulated (i.e., deep encoding with repetition and shallow processing without repetition). Our results revealed age-related deficits in new item identification for both the abstract figures and the non-words. Moreover, as indicated by the discriminability indexes $\left(\mathrm{A}^{\prime}\right)$, the young were better in discriminating the drawn and studied abstract figures. In line with previous research, this was likely due to the elderly making more false alarms (Bowman \& Dennis, 2015; Fraundorf, Hourihan, Peters, \& Benjamin, 2019). Partly in 
contrast to our expectations, age-related slowing in processing speed was only evident with the abstract figures but not with the non-words.

In addition, our results revealed that recognition performance was mediated by memory strength in both age groups to a similar extent. Namely, new item identification was superior to the recognition of the studied items when abstract figures and non-words were encoded shallowly. In contrast, the recognition of the new items was comparable to the semantically processed nonwords but was worse for the drawn abstract figures. Finally, our overall results were in line with our age-independent expectation concerning the memory advantage of deeper LOP and repetition over shallow LOP without repetition (Cherry et al., 2008; Craik, 2002; Craik \& Lockhart, 1972; Craik \& Tulving, 1975; Newell \& Andrews, 2004).

\section{IV.5.1. Age effects on new item recognition}

The elderly in the current study could identify the new abstract figures and non-words as 'new' less accurately than the young. Accordingly, the elderly produced more false alarms, which explains the age-related deficits in the differentiation of these stimuli. Several accounts can be offered in explanation of these results, such as the misrecollection account of cognitive aging (Dodson, Bawa, \& Krueger, 2007), age-dependent impairment in novelty assessment (Bastin, Delhaye, Moulin, \& Barbeau, 2019; Daffner et al., 2011; de Chastelaine, Mattson, Wang, Donley, \& Rugg, 2017), and decreased sensitivity to novelty (Czigler, Pato, Poszet, \& Balazs, 2006).

First, it is well-documented that during discrimination elderly can have difficulties with correct identification of new items when the old items are perceived as insufficiently distinct perceptually or conceptually from the new ones (Dodson, Bawa, \& Krueger, 2007; Fraundorf, Hourihan, Peters, \& Benjamin, 2019; Gallo, Cotel, Moore, \& Schacter, 2007). As such, new items can prompt an 
increase in the number of false alarms (Gallo, Cotel, Moore, \& Schacter, 2007; Kroll, Knight, Metcalfe, Wolf, \& Tulving, 1996). False memories can reliably induce misrecollection, which the aged are specifically prone to (Dodson, Bawa, \& Krueger, 2007).

Second, one of the most prominent accounts of novelty processing is the comparator or match/mismatch model (Kumaran \& Maguire, 2007). According to this model, the input undergoes a preliminary evaluation followed by the initiation of match/mismatch signals. If the stimulus matches an existing representation, it is recognized as familiar. On the other hand, novelty is detected if there is no match or if a mismatch is found (Kafkas \& Montaldi, 2014; Kafkas \& Montaldi, 2018; Kumaran \& Maguire, 2007). This comparison is assumed to be carried out by the hippocampus (Kafkas \& Montaldi, 2014; Kafkas \& Montaldi, 2018; Kumaran \& Maguire, 2007), a structure that is known to deteriorate with age (Bowman \& Dennis, 2015; de Chastelaine, Mattson, Wang, Donley, \& Rugg, 2017; Grady, McIntosh, \& Craik, 2003). Therefore, it is less surprising that novelty assessment of pre-experimentally unfamiliar stimuli could be demanding for the elderly. In other words, a less operational hippocampus, the lack of semantic support, and pre-experimentally existing stimulus representation could conjointly contribute to the evidenced age effects. Also, considering the meaningless nature of the stimuli, it seems plausible that the aged could rely less on their accumulated semantic knowledge to support the generation of the mismatch signals (Fraundorf, Hourihan, Peters, \& Benjamin, 2019; Zacks \& Hasher, 2006).

Third, as a consequence of cognitive aging, sensitivity to novelty may decline (Czigler, Pato, Poszet, \& Balazs, 2006; Daffner et al., 2006; Daffner et al., 2011). For example, it has been shown by Daffner et al. (2006) that cognitively average performing elderly had shorter viewing time in response to the novel stimuli than the elderly with high cognitive performance. The authors attributed this to decreased novelty sensitivity. Similarly, Czigler, Pato, Poszet, and Balazs 
(2006) also reported decreased sensitivity to visual novelty and overall cognitive slowing in the aged compared to the young. Therefore, it could be that the results of the current experiment may also reflect an age-related decrease in sensitivity to novelty.

\section{IV.5.2. Age effects on old item recognition}

We anticipated detecting age effects concerning the correct recognition of the studied but not the drawn abstract figures and semantically processed nonwords. When looking at the pure hit rates, age effects were not apparent. However, clear age effects were found concerning the discriminability indexes $\left(\mathrm{A}^{\prime}\right)$ of these stimuli, which involve false alarms. Namely, it was more difficult for the elderly to differentiate the drawn and studied abstract figures. Interestingly, this was not found for the non-words. Different theories have been proposed to explain memory performance in aging. Here, we will relate our findings to the processing theory account of cognitive aging (Craik, 2002), the environmental support hypothesis (Craik, 1986). the impaired perceptual encoding hypothesis (Trahan, Larrabee, \& Levin, 1986), and the self-initiated processing theory (Bridger et al., 2017; Fraundorf, Hourihan, Peters, \& Benjamin, 2019; Naveh-Benjamin \& Mayr, 2018).

First, according to the processing theory account of cognitive aging, we should have found smaller or no age effects on the deeply encoded (drawn and semantically processed) than shallowly encoded (studied) items (Craik, 2002), which was not the case. Therefore, this theory cannot explain the age effects on the processing of abstract figures and non-words. Interestingly, we found age effects for both types of abstract figures but not the non-words. These findings align with previous research using similar items (Badham \& Maylor, 2011; Harker \& Riege, 1985; Smith, Park, Cherry, \& Berkovsky, 1990). At the same time, these results also support the environmental support hypothesis (Craik, 1986). 
Regarding the abstract figures, it seems likely that the simple line drawings could not provide enough perceptual details to act as environmental support for the elderly. This explains why age effects are not found with complex, rich but are detected with simple pictorial materials (Harker \& Riege, 1985; Smith, Park, Cherry, \& Berkovsky, 1990). In this case, the complexity of the stimulus can be seen as environmental support. Regarding the lack of age effects with the nonwords, it seems likely that the age-related impairment diminished due to the rhyming words acting as environmental support. Indeed, Craik and McDowd (1987) did not find age differences in the recognition accuracy of target words encoded with cue phrases. Thus, it can be seen that age effects differ for verbal and visual materials. It also seems likely that environmental support is more powerful than mnemonic instructions for reducing age effects.

Second, the detected age effects on the discriminability of the drawn and studied abstract figures in the current experiment align with the age-related impairment in the perceptual encoding hypothesis (Trahan, Larrabee, \& Levin, 1986). This hypothesis holds that whereas young people can efficiently encode and retrieve rich perceptual information of both concrete and abstract figurative stimuli, the aged experience difficulties regardless of the nature of the stimuli. This is not surprising considering that visual processing declines with age (Bowman \& Dennis, 2015; Polidori, Zen, Zaccheo, \& Amenta, 1993). Thus, it seems that these factors can account for the age differences of abstract figurative memory. In agreement with previous research, it is also plausible that these results are due to the higher false alarm rates, and thus, some confusion evident with the elderly (Bowman \& Dennis, 2015; Fraundorf, Hourihan, Peters, \& Benjamin, 2019).

Third, we argue that the self-initiated processing theory account of cognitive aging cannot explain age-related deficits related to abstract figurative memory considering the marked difference in the A' scores (Bridger et al., 2017; Fraundorf, Hourihan, Peters, \& Benjamin, 2019; Naveh-Benjamin \& Mayr, 2018). 
As such, our results are agreeable with those by Fraundorf, Hourihan, Peters, and Benjamin (2019). This meta-analysis found larger age differences for tasks that required the participants to use deep, semantic encoding, implying preexperimentally familiar stimuli. Thus, it seems that aging negatively affects the memory of pre-experimentally familiar as well as pre-experimentally unfamiliar figurative items.

Finally, the setback in processing speed with the abstract figures complies with well-documented age-dependent cognitive slowing (Levin, Eisenberg, \& Benton, 1992; van Hooren et al., 2007). Interestingly, both the aged and the young reacted at a comparable pace to the non-words. A possible explanation could be that pictures are represented as integrated patterns, whereas verbal stimuli are not (Rajaram, 1996). Moreover, Noldy, Stelmack, and Campbell (1990) suggested that the processing of verbal stimuli is automatic and fast. In contrast, picture processing requires additional allocation of attentional resources, which can slow down reactions. Automated memory processes in healthy aging are preserved and comparable to that in young (Titov \& Knight, 1997). Consequentially, this can explain why the processing speed of the abstract figures did show age effects, and that of the non-words did not. Since cognitive slowing was found to be differential depending on the pre-experimentally unfamiliar stimulus being figurative or verbal, it seems plausible that age differences do not stem from a single global deficit (Benjamin, 2010; Fraundorf, Hourihan, Peters, \& Benjamin, 2019). 
IV.5.3. No age effects on the recognition performance as a function of memory strength

As expected, we found that stronger memories achieved by deeper LOP and repetition improved recognition accuracy and decreased RT-s of the drawn/semantically processed items as opposed to the studied ones in both age groups. These findings are in line with the notion that deeper LOP improves recognition and discrimination performance (Hulstijn, 1997; Paivio \& Desrochers, 1981; Solso, 1995). Furthermore, correct identification of the new items was superior when contrasted with items relying on weak visual and verbal (shallow encoding). As such, the current data show that it was likely more difficult to correctly recognize the shallowly encoded (studied) items as old. Apparently, the studied items were more likely perceived as 'new' than 'old'. Indeed, the RT-s of the studied and new stimuli were not significantly different. Thus, it seems that recognition of items without recent memory (new stimuli) is more effective compared to the recognition of stimuli relying on weak memories for both the young and elderly.

In contrast, recognition memory of the drawn figures was superior to the new ones. Also, the semantically processed non-words were recognized as 'old' just as well as the new ones were recognized as 'new'. The differential effect of LOP and repetition on the discrimination performance indicates a mediating effect of memory strength, which seems to withstand cognitive aging. One plausible explanation for the slightly different results on the drawn abstract figures and semantically processed non-words vs. new items could be the dualcoding theory of the images (Paivio, 1971, 2007). However, considering the meaningless nature of the abstract figures, it is unsure to what extent verbal codes could be created (Voss \& Paller, 2009). Therefore, task-induced differences in LOP could provide an alternative explanation. Previous research has demonstrated that drawing compared to writing (Wammes, Meade, \& Fernandes, 2016) or phonological encoding produces deeper processing, and establishes stronger 
memories (Matlin, 2005). This is due to the fact that the processing of the images requires pattern recognition (Craik, 2002), an ability that is also known to be preserved in the elderly (Titov \& Knight, 1997).

In conclusion, the current results demonstrate that aging impairs correct recognition of the new abstract figures and non-words. This was due to the elderly being prone to making more false alarms. Our findings further showed age-related impairments in recognition of the shallowly encoded abstract figures. No age differences were found for the deeply encoded abstract figures and nonwords. Thus, the current study further supports the notion that recognition performance in aging is only impaired under certain conditions (Fraundorf, Hourihan, Peters, \& Benjamin, 2019). 
V. Chapter:

The antimuscarinic Agent, Biperiden selectively impairs Memory of the Abstract Figures without affecting the Processing of Non-Words

Toth, M., Sambeth, A., Blokland, A. (2021). The antimuscarinic agent biperiden selectively impairs recognition of abstract figures without affecting the processing of non-words. Human Psychopharmacology: Clinical and Experimental, e2819. https://doi.org/10.1002/hup.2819
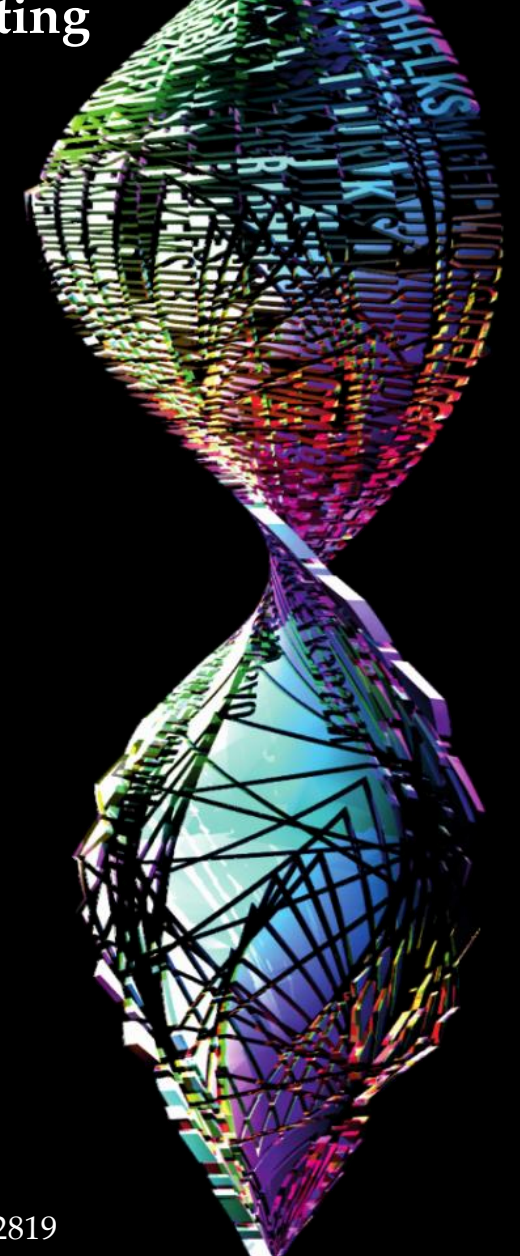


\section{V.1. Abstract}

\section{Objectives}

The present study investigated the effects of biperiden, a muscarinic type 1 antagonist, on the recognition performance of pre-experimentally unfamiliar abstract figures and non-words in healthy young volunteers. The aim was to examine whether $4 \mathrm{mg}$ biperiden could model the recognition memory impairment seen in healthy aging.

\section{Methods}

A double-blind, placebo-controlled, 2-way cross-over study was conducted. We used a three-phase (deep memorization, shallow memorization, and recognition) old/new discrimination paradigm in which memory strength was manipulated. Strong memories were induced by deep encoding and repetition. Deep encoding was encouraged by redrawing the abstract figures and mentioning existing rhyme words for the non-words (semantic processing). Weak memories were created by merely instructing the participants to study the stimuli (shallow memorization).

\section{Results}

Biperiden impaired recognition accuracy and prolonged reaction times of the drawn and the studied abstract figures. However, participants were biased towards 'old' responses in the placebo condition. The recognition of the new abstract figures was unaffected by the drug. Biperiden did not affect the recognition of the nonwords.

\section{Conclusions}

Although biperiden may model age-related deficits in episodic memory, the current findings indicate that biperiden does not mimic age-related deficits in recognition performance. 
Keywords: biperiden; recognition memory; abstract pictures; non-words; healthy aging; cognitive aging models 


\section{V.2. Introduction}

It is well established that healthy aging is associated with memory impairments. However, the effect of aging on memory seems to depend on which memory functions are being investigated. For example, aging seems to impair episodic memory most consistently, whereas semantic memory, working memory, and procedural memory remain to a great extent intact in healthy elderly (Nilsson, 2003). Furthermore, age-related impairments are typically found in recognition memory tests (Fraundorf, Hourihan, Peters, \& Benjamin, 2019; Rhodes, Greene, \& Naveh-Benjamin, 2019). In recognition memory paradigms, participants must recognize previously studied stimuli as 'old' correctly and identify not presented ones as 'new' (Malmberg, 2008).

However, the aging effect on recognition memory seems to depend on the stimulus's nature (i.e., identifying a stimulus as 'old' or 'new'). Age appears to decrease stimulus discriminability (Fraundorf, Hourihan, Peters, \& Benjamin, 2019; Wolk et al., 2009), which is typically related to a tendency to judge presented stimuli as 'old' despite them being new (Gallo, Cotel, Moore, \& Schacter, 2007; Kroll, Knight, Metcalfe, Wolf, \& Tulving, 1996). It seems likely that these performance differences are at least partly due to an impairment in sensitivity to novelty (Czigler, Pato, Poszet, \& Balazs, 2006; Daffner et al., 2006; Daffner et al., 2011). Another factor could be the limited availability of processing resources in older age (Park \& Festini, 2017). A final factor could be the agereaéted slowing in processing speed (Levin, Eisenberg, \& Benton, 1992; van Hooren et al., 2007). Salthouse (1996) proposed that this reduction in processing speed contributes to delayed cognitive process execution and the loss of information processed at earlier stages.

In addition to novelty processing, the level of processing (LOP) also seems to affect recognition performance in aged people (Fraundorf, Hourihan, Peters, \& Benjamin, 2019). The LOP theory predicts that deep (e.g., via 
mnemonics, meaning-extraction, pattern recognition, activation of prior knowledge) and intermediate processing (e.g., phonetics) lead to superior and faster retrieval when compared to shallow processing (e.g., perceptual analyses, rehearsal) (Craik, 2002; Craik \& Lockhart, 1972; Craik \& Tulving, 1975; Newell \& Andrews, 2004). Fraundorf, Hourihan, Peters, and Benjamin (2019) reported that age differences were larger when deep semantic encoding was applied compared to shallow processing. This may be related to age-related difficulties with selfinitiation of deep encoding strategies. Thus, when such strategies are provided age differences were not found (Craik \& Rose, 2012; Froger, Taconnat, Landre, Beigneux, \& Isingrini, 2009; Logan, Sanders, Snyder, Morris, \& Buckner, 2002).

In previous studies, it has been shown that selective blocking of the muscarinic type 1 (M1) receptors specifically impairs episodic memory (Borghans, Sambeth, \& Blokland, 2020; Borghans, Blokland, \& Sambeth, 2017; Sambeth, Riedel, Klinkenberg, Kahkonen, \& Blokland, 2015; Vingerhoets et al., 2017; Wezenberg, Verkes, Sabbe, Ruigt, \& Hulstijn, 2005). In these studies, it was found that the M1 antagonist biperiden (BIP) impaired the performance in the verbal learning task (VLT) but did not affect working memory, as measured by the n-back task. These effects appeared to be selective memory impairments since BIP treatment did not affect the performance in attention tasks. These results suggest that BIP treatment could be a suitable pharmacological model of agerelated episodic memory impairment.

Characterizing BIP's effects can aid a better understanding of which neurotransmitter systems may underlie the age-related memory deficits. This is relevant from a scientific viewpoint, and it may be relevant for the development of treatments for age-related memory deficit. This could be an M1 agonist such as BIP. To further investigate the validity of BIP as a pharmacological model of age-related memory impairment, we examined the effect of BIP on old/new discrimination performance using pre-experimentally unfamiliar stimuli in a sample of healthy young participants. We applied a three-phase old/new 
discrimination memory paradigm with abstract figures and non-words (Toth, Sambeth, \& Blokland, 2021). Memory strength was manipulated as a function of LOP (Craik, 2002; Craik \& Lockhart, 1972; Craik \& Tulving, 1975; Newell \& Andrews, 2004) and repetition (Hintzman \& Curran, 1997; Ranganath \& Rainer, 2003). Repetition is known to strengthen memory by increasing the subjective sense of familiarity resulting from the re-encoding of a particular memory trace (Hintzman \& Curran, 1997; Ranganath \& Rainer, 2003). In the current experiment, we first familiarized the stimuli using mnemonics to induce deep processing (deep memorization): the participants were asked to redraw the abstract figures and to mention existing rhyming words for the non-words (semantic processing). In the second phase, participants were asked to merely study the stimuli (shallow memorization). Here, the previously deeply encoded items were shown again in combination with some new items. Finally, an old/new recognition test was applied in which stimuli from the first and second phases were intermixed with new ones. Both recognition accuracy and speed were assessed.

Based on previous studies in healthy aging, we did not anticipate detecting drug effects on the overall correct old item recognition (drawn/semantically encoded and studied items). However, we anticipated lower discriminability indexes due to higher false alarm rates (incorrectly identifying new items as 'old'), and slower reaction times as a consequence of drug treatment. Furthermore, we anticipated that BIP would decrease the number of correctly rejected new items. Also, we expected that BIP would increase the false alarm rates in response to the new stimuli presented only during the recognition phase. Finally, we hypothesized that in the BIP as well as the placebo (PLA) sessions, deep memorization and repetition would prompt better recognition than shallow memorization without repetition. In other words, items relying on strong memory would be better recognized than those relying on weak memory. 


\section{V.3. Methods}

\section{V.3.1. Participants}

Based on previous studies using the current paradigm, an a priori statistical power analysis using $G^{*}$ power 3.1 showed that in order to detect significant behavioral effects using an ANOVA, 19 participants were required with an effect size of 0.4 and power of at least $90 \%$ at a significance level of $5 \%$ (Faul, Erdfelder, Lang, \& Buchner, 2007). Therefore, 21 healthy volunteers between the age of 18 and 35 years were recruited. One participant terminated the study due to personal reasons, and thus, was excluded from further analyses. The final dataset contains 20 participants (five males, with a mean age of 23 years) who were students from Maastricht University, with the highest education level being pre-university education or bachelor's degree. Inclusion was based on medical screening, which involved filling in a medical questionnaire followed by a detailed examination by a physician. Blood and urine tests were taken to confirm the participants' health condition and to rule out the apparent use of psychoactive drugs (e.g., cannabinoids, methylphenidate, cocaine, amphetamine, antidepressants, etc.), pregnancy or lactation. Furthermore, participants were included if their body mass index fell within the range of $18.5-30 \mathrm{~kg} / \mathrm{m} 2$.

Participants were excluded in case of hypersensitivity to any component of the formulation of BIP or related compounds. Further exclusion criteria comprised smoking, excessive drinking (> 20 glasses of alcohol-containing beverages a weak), use of medication other than oral contraceptives, and any sensory or motor deficits, which could have affected test performance. Participants with neurologic, cardiovascular, pulmonary, hepatic, renal, metabolic, gastrointestinal, or endocrine diseases were also ruled out. Additionally, participants were excluded if they had a history of psychiatric 
conditions, such as ADHD, schizophrenia, different forms of depression, anxiety, mood and personality disorders, or addiction.

This study was conducted according to the codes of ethics on human experimentation established by the declaration of Helsinki (1964) and amended in Fortaleza (2013), and in accordance with the Medical Research Involving Human Subjects Act (WMO). The Medical Ethics Committee approved the study of the University Hospital Maastricht and Maastricht University. Medical Ethical Approval Code: EPU-P95A NL58970.068.16. Each participant received monetary compensation or research participation credit points.

\section{V.3.2. Study Design and Medication}

A randomized, double-blind placebo (PLA) controlled 2-way crossover design was applied with a counterbalancing of orders over the two sessions. This means that each participant was tested two times on two separate occasions, once receiving $4 \mathrm{mg}$ BIP (Akineton ${ }^{\circledR}$ ) and once PLA. The order of treatment (PLA-BIP and BIP-PLA) was balanced in the sample. The washout period was 7-14 days. The order of the medications was blinded. Treatment was applied in accordance with previous results showing that peak plasma levels of BIP are reached 60-90 min after intake of a single dose (Sudo et al., 1998).

\section{V.3.3. Procedure}

Volunteers signed an informed consent before the medical examination. Hereafter, they received training to be familiarized with the test procedures. A test battery was used during this training session, which contained a different set of stimuli from those used during the actual test days. This was done to avoid learning effects. Hereafter, the test days were scheduled within a maximum of 
seven days after the training session. The two testing days were scheduled at the exact same time of the day to reduce diurnal effects.

Before and after the testing sessions, participants filled in questionnaires assessing their general well-being status and possible complaints (e.g., headache, drowsiness, sweating, sleepiness). Participants had to indicate whether they experienced any of the 33 possible complaints on a four-point scale. For example, a score of zero stood for "I do not experience this complaint at all," and a three stood for "I am experiencing this complaint strongly". If the participants experienced any complaints not listed on the questionnaire, they were asked to mention them on the questionnaire form in writing. Scores were compared between the different time points to examine treatment-induced side effects. Adverse events were monitored using printed forms.

Subsequently, 90 min before the behavioral testing, medication (BIP or PLA) was administered. The participants were asked to refrain from alcohol, smoking, and caffeine $12 \mathrm{~h}$ before testing and not to use drugs throughout the study.

A memory paradigm with abstract figures and non-words was applied in separate tests (Toth, Sambeth, \& Blokland, 2021). See Fig. 1 for an example of the stimuli used. Every participant performed each test phase first with the abstract figures and then with the non-words to minimize the verbalization of the figurative stimuli. The experiment consisted of three phases (see Fig. 2). In phase 1 (deep memorization leading to 'strong' memory), participants were familiarized with a series of 15 monosyllabic abstract figures or non-words in separate tests (list 1: L1). Participants were asked to manually redraw the abstract figures on an answer sheet to induce deep LOP. They had to mention existing English or Dutch rhyming words for each non-word to induce intermediate LOP. Stimuli were presented for $1 \mathrm{~s}$, and the participants were given $14 \mathrm{~s}$ to execute the mnemonic encoding task. If they were ready earlier, they could press a button, and $2 \mathrm{~s}$ later, the next stimulus appeared. Stimuli were extracted from previous 
studies (Glosser, Friedman, Grugan, Lee, \& Grossman, 1998; Redoblado, Grayson, \& Miller, 2003; Seidenberg, Plaut, Petersen, McClelland, \& McRae, 1994).

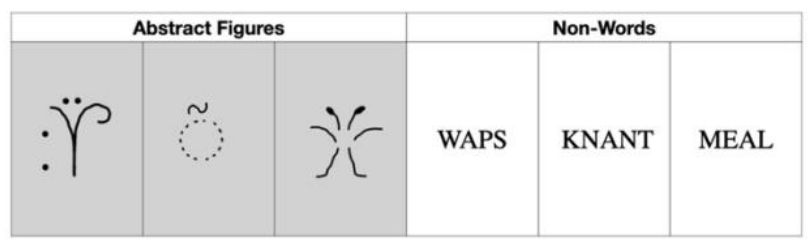

Fig. 1: Examples of the stimuli used.

During phase 2 (shallow memorization leading to 'weak' memory), participants were instructed to remember as many stimuli as possible. In this phase, 30 stimuli (abstract figures or non-words) were used: 15 stimuli from L1 were randomly mixed with 15 new ones (L2). All stimuli were shown for $1 \mathrm{~s}$ with an inter stimulus interval (ISI) of $2 \mathrm{~s}$. 


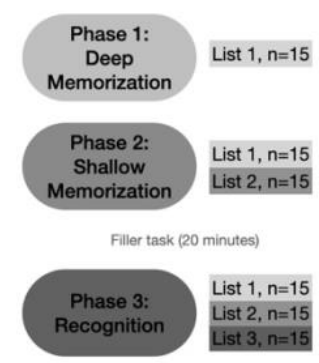

Fig. 2: Schematic overview of the experimental design. Phase 1: deep memorization with the preexperimentally unfamiliar abstract figures and non-words in separate tests using a mnemonic encoding task (redrawing the abstract figures and mentioning rhyming words for the non-words). The 15 stimuli used here form List 1 (drawn/semantically processed stimuli). Phase 2: shallow memorization with the instruction to remember as many stimuli as possible. This phase contained items from List 1 and 15 new ones (List 2, studied stimuli). Phase 3: recognition of the stimuli including List 1, List 2, and 15 new (List 3). n: number of stimuli presented.

During phase 3, participants were asked to decide if they had seen the presented stimulus in the previous series (L1 and L2) or whether the stimulus was new to them (L3: new, $\eta=15$ ). The 45 non-words or abstract figures were presented for a duration of $1 \mathrm{~s}$, or less in case of faster button press; the ISI was 2.5 s. Participants had to press the corresponding buttons ('old' for L1 and L2, or 'new' for L3 stimuli) on a response box as quickly and accurately as possible.

The Attention Network Test was administered between phase 2 and 3 as a filler task lasting 20 minutes (Togo, Lange, Natelson, \& Quigley, 2015). 


\section{V.3.4. Data Analysis}

Before analysis, all data were evaluated for having normal distribution and homogeneity of variance. Also, raw data were checked for outliers. Outlier values were replaced with their regression estimates produced by the Missing Value Analyses (IBM SPSS Statistics for Macintosh, Version 27.0. Armonk, NY: IBM Corporation). Additionally, due to technical issues, 1-2 responses per participant were missing (e.g., the button press was not recorded). In these cases, values were replaced with their regression estimates. Effect sizes are reported based on partial eta-squared $\left(\eta p^{2}\right)$ data. Furthermore, Mauchly's Test of Sphericity was applied. In case the assumption of sphericity was violated, a Greenhouse-Geisser correction was used. In all cases, degrees of freedom of assumed sphericity are reported. Post-hoc comparisons and simple effects were investigated using paired-samples t-tests, applying adjustments for multiple comparisons; the observed p-values were multiplied by the number of comparisons, which was tested against the set significance level of 0.05 .

For the behavioral data, Signal Detection Theory (SDT) was applied in order to investigate the discrimination performance (Benjamin \& Bawa, 2004; Benjamin, Diaz, \& Wee, 2009; Stanislaw \& Todorow, 1999; Verde \& Rotello, 2007). Discrimination accuracy was defined as the ability to distinguish the different types of stimuli (drawn/semantically processed, studied, and new). Correct responses included an 'old' response to the drawn/semantically processed items, and the studied stimuli, and a 'new' response to the new items. Incorrect responses involved a 'new' response to the drawn/semantically processed items and the studied stimuli and an 'old' response to the new stimuli. See Table 1 for an overview. 
Table 1: Overview of the different types of responses as a function of stimulus type.

\begin{tabular}{ccc}
\hline & Stimulus Type & Response \\
\hline Hit $(\mathrm{H})$ & $\begin{array}{c}\text { Drawn or semantically } \\
\text { processed/Studied }\end{array}$ & 'Old' \\
\hline Miss (M) & $\begin{array}{c}\text { Drawn or semantically } \\
\text { processed/Studied }\end{array}$ & 'New' \\
\hline Correct Rejection (CR) & New & 'New' \\
\hline False Alarm (FA) & New \\
\hline Hit Rate (HR) & Drawn or semantically & H/(H + M) \\
\hline porrect Rejection Rate (CRR) & New & $\mathrm{CR} /(\mathrm{CR}+\mathrm{FA})$ \\
\hline
\end{tabular}

Given the memory strength manipulation in the current design (deep memorization, shallow memorization and recognition), the correct response rates, being hit rates (HR) for the drawn/semantically processed and the studied items and correct rejection rates (CRR) for the new, were used to evaluate the discrimination accuracy. Furthermore, in order to investigate discriminability, non-parametric $\mathrm{A}^{\prime}$ statistics were computed for the drawn/semantically processed and the studied stimuli using Equations (1) or (2) (Snodgrass \& Corwin, 1988; Stanislaw \& Todorow, 1999). A'varies from 0 to 1 with 0.5 indicating chance performance. Higher values are indicative of improved performance (Snodgrass \& Corwin, 1988; Stanislaw \& Todorow, 1999).

$$
\begin{aligned}
& \mathrm{A}^{\prime}=0.5+\frac{(H R-F A R)(1+H R-F A R)}{4 H R(1-F A R)}, \text { if } H R \geq F A R \\
& \mathrm{~A}^{\prime}=0.5-\frac{(H R-F A R)(1+H R-F A R)}{4 H R(1-F A R)}, \text { if } H R<F A R
\end{aligned}
$$

$\mathrm{A}^{\prime}$ : discriminability index, HR: hit rate, FAR: false alarm rate.

During recognition, the a priori probabilities of old and new items and the quality of the match between a test item and the memory for studied items can influence the bias parameter (Huang \& Ferreira, 2020; Stanislaw \& Todorow, 
1999). Such a model does not fit the current paradigm due to the memory strength manipulation used and the equivalent proportion and intended comparison of the drawn/semantically processed $(\eta=15)$, studied $(\eta=15)$, and new items $(\eta=$ 15) (Benjamin \& Bawa, 2004). After all, the final proportion of 'old' and 'new' responses was 2:1. Therefore, we calculated the total amount of 'old' (H+FA) and 'new' $(\mathrm{M}+\mathrm{CR})$ responses given by the participants. This was done to examine whether there was a preference for either the 'old' or 'new' responses. Results were compared using paired samples t-tests with Bonferroni corrections.

RT data of the hits were evaluated, as well. To be able to use parametric tests, RT-s were transformed into $|\log (1 / \mathrm{RT})|$ to obtain a normal distribution of the data (Osborne, 2002). Moreover, the median RT data are reported as central tendency parameters, together with the corresponding first and third interquartile ranges (Ratcliff, 1993).

Statistical analysis was conducted using SPSS 27.0. A repeated measures analysis of variance (ANOVA) was used to investigate the recognition accuracy scores and RT-s for the different treatments and types of stimuli in the different categories as assessed in Phase 3. Thus, the within-subject variables for the abstract figures and the non-words were treatment (BIP and PLA), and stimulus type (drawn/ semantically processed, studied, and new items). Finally, treatment effects per stimulus type were evaluated using individual t-tests, which were corrected for multiple comparisons.

\section{V.4. Results}

Although there was an unequal number of old-responses over newresponses (2:1), we found that there was no response bias towards old-responses. However, the participants made more old-responses and less new-responses in case of the abstract figures in the PLA sessions $(p<.001$; see Table 2). 
Additionally, there were hardly any missing responses in the BIP (abstract figures: $3.6 \%$, non-words: $1.56 \%$ ) and in the PLA (abstract figures: $0.4 \%$, nonwords: $1.4 \%)$ session.

Table 2: The total number of old and new responses during the recognition test. Data represent the means and the standard deviations of the total old- and new responses and the corresponding \% compared to the 90 items/stimulus category (abstract figures and non-words), and the t-statistics.

\begin{tabular}{lcccc}
\hline \multicolumn{2}{c}{ Placebo } & \multicolumn{2}{c}{ Biperiden } \\
\hline & Abstract Figures & Non-words & Abstract Figures & Non- words \\
& & & \\
\hline Old & $27.60(3.21)$ & $23.25(6.13)$ & $21.95(5.91)$ & $23.20(3.55)$ \\
\hline New & $17.20(3.17)$ & $21.10(5.91)$ & $21.45(4.99)$ & $21.10(4.12)$ \\
\hline T-test & $t(19)=7.32$, & $t(19)=0.22$, & $t(19)=2.15$, & $t(19)=2.10$, \\
& $p<.001$ & $p<.830$ & $p>.431$ & $p>.224$ \\
& & & & \\
\hline
\end{tabular}

\section{V.4.1. Abstract Figures}

When analyzing the accuracy scores (HR and CRR) in the session with the abstract figures the ANOVA revealed a main effect of treatment $[F(1,19)=$ 7.44, $\left.\eta p^{2}=0.28, p<.013\right]$ and stimulus type $\left[F(2,38)=66.02, \eta p^{2}=0.78, p<.001\right.$; see Fig. 3]. Moreover, the interaction term treatment $\mathrm{x}$ stimulus type was also significant $\left[F(2,38)=10.20, \eta p^{2}=0.35, p<.003\right.$; see Fig. 3]. Simple effects analyses revealed that BIP compared to the PLA impaired correct recognition of the drawn $[t(19)=3.26, p<.012]$ and studied $[t(26)=3.24, p<.012]$ but not the new abstract figures $[t(19)=1.91, p>.210]$. 
A

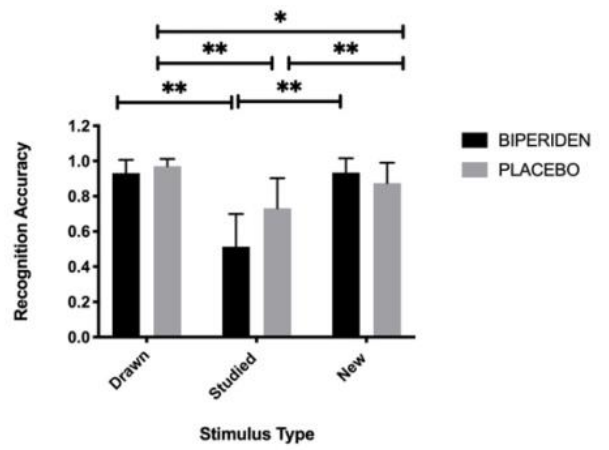

B

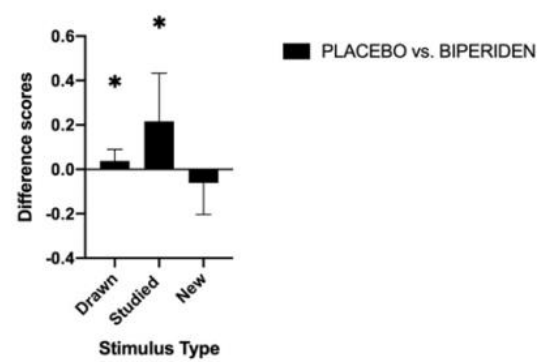

Fig. 3: Recognition accuracy of the abstract figures according to treatment and stimulus type. The bars represent the means with the standard deviations.

A) Stimulus type effects shown as the proportion of the correct responses: hit rates for the drawn and the studied, and correct rejection rates for the new abstract figures after placebo and biperiden treatment.

B) Treatment effects depicted as the difference scores per stimulus type (drawn, studied, and new).

${ }^{* *} p<.001,{ }^{*} p<.05$

The analyses with respect to stimulus type showed that in the sessions with BIP, participants could more accurately identify the drawn than the studied $[t(19)=7.70, p<.001$; see Table 3], and the new than the studied items $[t(19)=6.28$, $p<.001$; see Table 3]. No such difference was detected between the drawn and new abstract figures $[t(19)=0.14, p>.999$; see Table 3]. The same analyses in the session with PLA revealed that participants could more accurately recognize the drawn stimuli compared to the weak $[t(19)=6.45, p<.001$; see Table 3] and compared to the new items $[t(19)=3.87, p<.003$; see Table 3]. Also, more new stimuli were correctly endorsed compared to the studied items $[t(19)=3.35, p<$ .009]. 
Table 3: Means and standard deviations of the signal-detection measures during the recognition of the abstract figures (drawn, studied, and new) after placebo and biperiden.

\begin{tabular}{clll}
\hline \multicolumn{3}{c}{ Abstract Figures } \\
\hline Stimulus Type & Parameters & \multicolumn{1}{c}{ Placebo } & \multicolumn{1}{c}{ Biperiden } \\
\hline Drawn & HR & $0.97(0.04)^{\text {aa }}$ & $0.93(0.08)^{\text {aa }}$ \\
\cline { 2 - 4 } & $\mathrm{A}^{\prime}$ & $0.82(0.09)^{\text {aa }}$ & $0.87(0.19)^{\text {aa }}$ \\
\hline Studied & HR & $0.73(0.17)$ & $0.51(0.19)^{*}$ \\
\cline { 2 - 4 } & $\mathrm{A}^{\prime}$ & $0.67(0.10)$ & $0.62(0.12)$ \\
\hline New & CRR & $0.87(0.12)^{\text {aa }}$ & $0.93(0.08)^{\text {a }}$ \\
\hline
\end{tabular}

HR: hit rate, CRR: correct rejection rate, $\mathrm{A}^{\prime}$ : discriminability index

Treatment effects ${ }^{*}: p<.05$; Different from studied stimuli: aa: $p<.001$, a: $p<0.05$; Different from new stimuli:

b: $p<.05$

The analyses performed on the $\mathrm{A}^{\prime}$ scores of the abstract figures resulted in a significant main effect of stimulus type $\left[F(1,19)=112.14, \eta p^{2}=0.86, p<.001\right.$; see Table 3]. Post-hoc tests showed that the participants could discriminate the drawn items more easily than the studied $(p<.001)$. Moreover, the treatment $\mathrm{x}$ stimulus type interaction was found to be significant $\left[F(1,19)=8.93, \eta p^{2}=0.032\right.$, $p<.008$; see Table 3]. Simple effects analyses revealed no treatments effects [ $t(19)$ $=1.81, p>.172 ; t(19)=1.67, p>.218$, respectively; see Table 3]. However, in both the PLA and the BIP session it was easier to discriminate the drawn than the studied items $[t(19)=6.58, p<.001 ; t(19)=9.35, p<.001$, respectively; see Table 3]. No main effect of treatment was found $\left[F(1,19)=0.02, \eta p^{2}=0.01, p>.893\right]$.

Concerning the reaction times, the ANOVA confirmed a significant main effect of treatment $\left[F(1,19)=9.11, \eta p^{2}=0.32, p<.007\right]$ and stimulus type $[F(2,18)=$ 68.69, $\left.\eta \mathrm{p}^{2}=0.88, p<.001\right]$. There was a treatment $\mathrm{x}$ stimulus type interaction detected $\left[F(2,18)=6.36, \eta p^{2}=0.41, p>.008\right.$; see Table 4]. Simple effects analyses revealed that BIP compared to the PLA slowed the reactions in response to the drawn $[t(19)=3.78, p<.003]$ and the studied $[t(19)=2.90, p<.027]$ but not to the new abstract figures $[t(19)=0.29, p>$.999]. Simple effects analyses with respect to stimulus type showed that in the sessions with BIP participants reacted faster to the drawn than the studied $[t(19)=8.23, p<.001]$, the new than the studied items 
$[t(19)=3.47, p<.009]$ and the drawn compared to the new abstract figures $[t(19)$ $=3.49, p<.009]$. Similarly, the same analyses in the session with PLA revealed that participants reacted faster to the drawn than the studied $[t(19)=8.99, p<.001]$ and new abstract figures $[t(19)=7.49, p<.001]$. No such difference was found between the studied and new items $[t(19)=0.60, p>.999]$.

Table 4: Median reaction times (middle 50\% range), and their corresponding first and third interquartile ranges (IQ) in milliseconds in response to the abstract figures (drawn, studied, and new) after placebo and biperiden treatment.

\begin{tabular}{ccc}
\hline & Abstract Figures Median (1-3 IQ) \\
\hline Stimulus Type & Placebo & Biperiden \\
\hline Drawn & $589(527-628)^{\text {ab }}$ & $633(609-751)^{a b *}$ \\
\hline Weak & $724(637-793)$ & $794(741-886)^{*}$ \\
\hline New & $718(649-760)$ & $733(636-800)^{a}$
\end{tabular}

Treatment effects: ${ }^{*}: p<.05$; Different from studied stimuli: aa: $p<.001$, a: $p<.05$; Different from new stimuli: b: $p<.05$.

\section{V.4.2. Non-Words}

The ANOVA analysis for the non-words revealed a main effect of stimulus type $\left[F(2,18)=32.51, \eta p^{2}=0.78, p<.001\right.$; see Fig. 4]. Post-hoc tests showed that the semantically processed stimuli were recognized better than the studied $(p<.001)$. Also, more new stimuli were endorsed correctly compared to the studied items $(p<.001)$. No such difference was found between the semantically processed and the new stimuli $(p>.780)$. Moreover, neither treatment $[F(1,19)=$ $\left.0.02, \eta p^{2}=0.01, p>.964\right]$ nor the interaction term treatment $\mathrm{x}$ stimulus type was statistically meaningful. $\left[F(2,38)=0.07, \eta p^{2}=0.01, p<.934\right]$. 

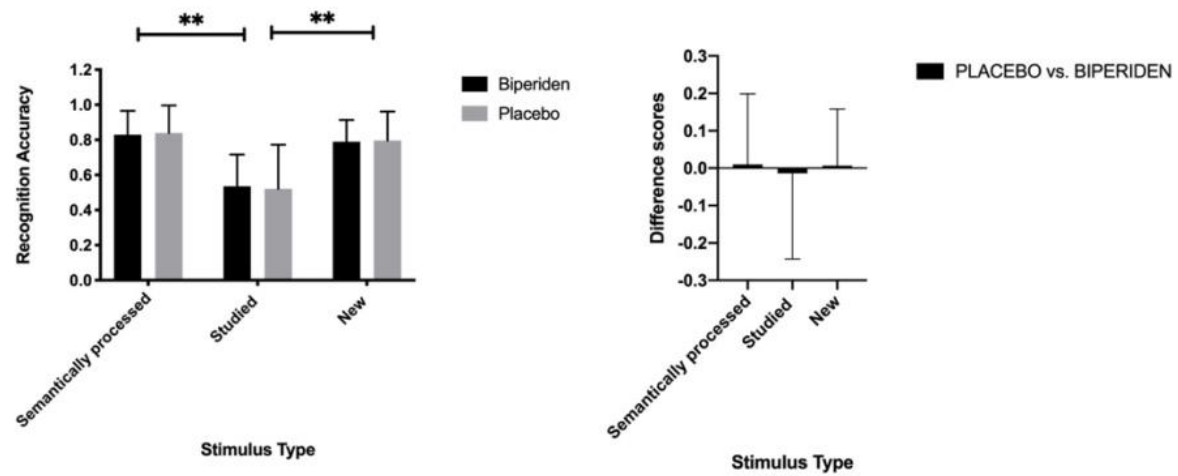

Fig. 4: Recognition accuracy of the non-words according to treatment and stimulus type. The bars represent the means with the standard deviations.

A) Stimulus type effects shown as the proportion of the correct responses: hit rates for the semantically processed and the studied, and correct rejection rates for the new abstract figures after placebo and biperiden treatment.

B) Treatment effects depicted as the difference scores per stimulus type (semantically processed, studied, and new).

** $p<.001$

The analyses of the signal detection derived measures of the non-word stimuli are presented in Table 5.

Table 5: Means and standard deviations of the signal-detection measures during the recognition performance of the non-words (semantically processed, studied, and new) after placebo and biperiden.

\begin{tabular}{clll}
\hline \multicolumn{2}{c}{ Non-Words } & \\
\hline Stimulus Type & Parameters & Placebo & Biperiden \\
\hline Semantically processed & HR & $0.84(0.16)^{\text {aa }}$ & $0.83(0.14)^{\text {aa }}$ \\
\cline { 2 - 4 } & $\mathrm{A}^{\prime}$ & $0.68(0.11)^{\text {aa }}$ & $0.68(0.11)^{\text {aa }}$ \\
\hline Weak & HR & $0.52(0.25)$ & $0.54(0.18)$ \\
\cline { 2 - 4 } & $\mathrm{A}^{\prime}$ & $0.57(0.17)$ & $0.56(0.07)$ \\
\hline New & CRR & $0.80(0.17)^{\text {aa }}$ & $0.79(0.12)^{\text {aa }}$
\end{tabular}

HR: hit rate, CRR: correct rejection rate, $\mathrm{A}^{\prime}$ : discriminability index

Different from studied stimuli: aa: $p<.001$ 
The analyses performed on the $\mathrm{A}^{\prime}$ scores resulted in a significant main effect of stimulus type $\left[F(1,19)=41.19, \eta p^{2}=0.68, p<.001\right.$; see Table 5]. Post-hoc tests showed that the participants could discriminate the semantically processed items more easily than the studied $(p<.001)$. Finally, neither the treatment $x$ stimulus type interaction $\left[F(1,19)=0.04, \eta p^{2}=0.02, p>.842\right.$; see Table 5] nor treatment was found to be significant $\left[F(1,19)=0.02, \eta p^{2}=0.01, p>.903\right]$.

The analyses of the reaction times yielded a main effect of stimulus type $\left[F(2,18)=4.45, \eta p^{2}=0.33, p<.027\right.$; see Table 6]. Post-hoc tests revealed that reactions to the semantically processed items were faster compared to the new ones $(p<.045)$. Finally, neither treatment $\left[F(1,19)=2.64, \eta p^{2}=0.12, p>.121\right]$ nor the interaction term treatment $\mathrm{x}$ stimulus type was statistically meaningful $\left[F(2,18)=0.35, \eta p^{2}=0.01, p>.966\right]$.

Table 6: Median reaction times (middle 50\% range; in milliseconds), and their corresponding first and third interquartile ranges in response to non-words (semantically processed, studied and new) after placebo and biperiden treatment.

\begin{tabular}{ccc}
\hline & Non-Words & \\
\hline Stimulus Type & Placebo & Biperiden \\
\hline Semantically processed & $628(592-682)^{\mathrm{b}}$ & $624(587-648)^{\mathrm{b}}$ \\
\hline Studied & $650(552-713)$ & $634(596-685)$ \\
\hline New & $698(627-762)$ & $669(589-727)$ \\
\hline
\end{tabular}

Different from the new stimuli: $\mathrm{b}: p<.05$

\section{V.4.3. Complaints and POMS}

The analyses did not result in any significant treatment effects for the neurovegetative complaints and the POMS [all associated $t$-values $<1.37, p>.330$; $t$-values $<1.61, p>.123$, respectively; see Table 7]. Also, no further complaints other than listed in the questionnaire were mentioned. There were no adverse events found. 
Table 7: Mean difference scores as change from baseline (standard deviations) for the questionnaire data. Negative numbers indicate a decrease and positive numbers indicate an increase in the subjective feeling.

\begin{tabular}{lcc}
\hline & Biperiden & Placebo \\
\hline Profile of mood states & $3.78(6.67)$ & $1.83(6.37)$ \\
\hline Depression & $0.9(2.63)$ & $-0.65(3.22)$ \\
\hline Tension & $-0.15(2.03)$ & $-0.10(1.40)$ \\
\hline Aggression & $0.75(2.53)$ & $0.95(2.69)$ \\
\hline Fatigue & $4.85(7.21)$ & $3.35(5.59)$ \\
\hline Vigor & & $0.10(0.55)$ \\
\hline Neurovegetative effects & $0.25(0.55)$ & $0.45(1.00)$ \\
\hline Headache & $0.60(1.14)$ & $0.50(1.00)$ \\
\hline Sleepiness & $0.50(1.00)$ & $0.10(0.31)$ \\
\hline Dizziness & $0.10(0.31)$ & $0.60(1.05)$ \\
\hline Nausea & $0.30(1.17)$ & $0.50(0.69)$ \\
\hline Dry mouth & $0.55(0.76)$ & $0.75)$ \\
\hline Fatigue & & \\
\hline Dlurred vision & & \\
\hline
\end{tabular}

\section{V.5. Discussion and Conclusions}

The present study aimed to examine whether BIP could model the recognition memory impairment as seen in healthy aging using an old/new recognition paradigm with abstract figures and non-words. The current results show that BIP impaired the correct recognition and slowed the abstract figures' reaction times. Interestingly, BIP only impaired the recognition of the drawn (deeply memorized and repeated items relying on strong memory) and studied (shallowly memorized and not repeated items relying on weak memory) figures 
but not the correct identification of the new abstract figures. Furthermore, the processing of the non-words was not affected by BIP treatment.

Based on the aging literature, we expected that BIP treatment would not affect the overall recognition performance of the drawn and studied (old) stimuli. However, the current data showed that the studied abstract figures were less well recognized after BIP treatment. However, since the drug only affected the processing of the abstract figures, it is possible that the effects were related to a response bias. Namely, we detected an 'old' response bias in the PLA session but not in the BIP session. Therefore, the response bias may underlie the observed drug effects on recognition memory. Further, although it was expected that BIP would decrease the discriminability index $\left(\mathrm{A}^{\prime}\right)$ of the drawn/semantically processed and studied items, the current data did not show this impairment for either the abstract figures or the non-words. However, as expected, BIP prolonged the reaction times when responding to the drawn and the studied abstract figures. Taken together, the effects of BIP did not fully model the typical age-related deficits in recognition performance.

The finding that BIP did not affect the recognition performance of the non-words is somewhat unexpected. The treatment effects were dependent on the type of stimulus used. It could be argued that the recognition performance of the abstract pictures was better than the non-words and that the high performance is more sensitive to treatment effects. However, the performance of the non-words was about $80 \%$ correct, which can also be considered relatively high. Moreover, the strongest treatment effects for the abstract figures were found for the studied stimuli. Here, the recognition performance was about $70 \%$ correct. Therefore, the lack of treatment effects for the non-words cannot be attributed to recognition performance level.

The lack of effect for the non-words may also be explained based on an age-related difference in the use of pre-existing semantic knowledge (Badham, Poirier, Gandhi, Hadjivassiliou, \& Maylor, 2016; de Chastelaine, Mattson, Wang, 
Donley, \& Rugg, 2017; Fraundorf, Hourihan, Peters, \& Benjamin, 2019). Belleville, Menard, and Lepage (2011) tested the discrimination performance of existing words vs. pseudo-words. They applied a two-phase study-recognition memory paradigm in young, healthy elderly, and MCI patients. Their results showed that the healthy elderly were impaired in recognizing existing words but that the performance on the pseudo-words was not affected. If aging does not affect the recognition of non-words, the current data may not dismiss the notion that BIP could be a model for recognition deficits in aging. It should be noted that in the study of Belleville et al., pseudo-words were only presented once, and no deep processing took place. In the current study, the non-words were deeply processed and repeated, which would make them more familiar than the pseudo words in the Belleville et al. study. Finally, it should be mentioned that BIP (2 mg) decreased correct recognition of words in the verbal learning task (Wezenberg, Verkes, Sabbe, Ruigt, \& Hulstijn, 2005). Although this is another recognition task using existing words, these data suggest that BIP could impair word recognition as seen in aging. Further studies are indicated in which the effects of BIP on the familiarity of words are tested.

The elderly often have difficulties identifying new items correctly when the old items are perceived as insufficiently distinct (Dodson, Bawa, \& Krueger, 2007; Fraundorf, Hourihan, Peters, \& Benjamin, 2019; Gallo, Cotel, Moore, \& Schacter, 2007). Consequently, new items are identified as 'old' in recognition tasks (i.e., more false alarms) (Gallo, Cotel, Moore, \& Schacter, 2007; Kroll, Knight, Metcalfe, Wolf, \& Tulving, 1996). The stimuli in the current experiment were pre-experimentally unfamiliar, which theoretically could make their discrimination more difficult than the pre-experimentally known items. Indeed, several empirical studies have shown that memory is worse for preexperimentally unknown vs. known items, such as unfamiliar vs. familiar symbols (Cycowicz \& Friedman, 2007), words vs. non-words (Belleville, Menard, \& Lepage, 2011; Gardiner \& Java, 1990). In agreement with these findings, BIP 
should decrease the number of correctly recognized new stimuli (abstract pictures and non-words). However, this was not observed in the current study, which further undermines the notion that BIP models recognition deficits in aging.

The drug-induced impairment in reaction times to the abstract figures complies with the well-documented age-dependent cognitive slowing (Levin, Eisenberg, \& Benton, 1992; Salthouse, 1996; van Hooren et al., 2007). A decrease in response times after BIP has also been observed in other tasks in previous results (Sambeth, Riedel, Klinkenberg, Kahkonen, \& Blokland, 2015; Silver \& Geraisy, 1995; Wezenberg, Verkes, Sabbe, Ruigt, \& Hulstijn, 2005). In addition, pictures are represented as integrated patterns (Rajaram, 1996), and their processing requires additional allocation of attentional resources, which can slow down reactions (Noldy, Stelmack, \& Campbell, 1990). If this was true, then BIP might have affected attention. However, this seems unlikely considering that participants did not report sedation in the present study.

Furthermore, our findings align with previous research. Firstly, the memory strength manipulation showed a clear difference between the deeply and shallowly processed stimuli (Hulstijn, 1997; Paivio \& Desrochers, 1981; Solso, 1995). Secondly, in the PLA condition, previous behavioral findings using this paradigm were replicated (Toth, Sambeth, \& Blokland, 2021). Namely, the correct identification of the new abstract figures and non-words was superior to old item recognition when they were merely studied and not repeated, but not when they were drawn or semantically processed. Finally, as in previous studies, $4 \mathrm{mg}$ BIP did not cause any adverse effects as measured by the POMS.

In closing, although BIP has been found to mimic an episodic memory impairment in young, healthy volunteers, the current data do not indicate that BIP can adequately model typical age-related deficits in recognition performance of abstract figures and non-words. 
As described in Chapter 1, it is not well understood which type of information, old or new, provides processing advantages. In particular, it is not known which factors might influence old/new recognition and how age might impact such processing. Our understanding of the processing of pre-experimentally unfamiliar stimuli, such as abstract figures and non-words, is even more limited. Such stimuli hardly rely on semantic knowledge, making it possible to isolate pure learning and memory effects. Thus, knowing how the brain processes and discriminates such stimuli can significantly improve our understanding of visual, lexical, and orthographic memory processing and contribute to establishing appropriate aging models.

This dissertation's overarching aim was to investigate how the brain processes and discriminates pre-experimentally unfamiliar stimuli and which factors affect such processing. One possible factor, the role of memory strength, was investigated. Furthermore, the impact of aging on recognition performance was examined. Additionally, a pharmacological recognition memory deficit model was investigated. This aim was addressed through specific research questions that were answered in the different experimental chapters. The first study examined whether pre-experimental familiarity affects recognition performance using pre-experimentally familiar (well-known figures and words) and pre-experimentally unfamiliar stimuli (abstract figures and non-words). Here, a paradigm was used in which memory strength was manipulated to test whether this factor affects recognition performance (Chapter II). Next, the underlying brain processes were investigated using the previously characterized behavioral paradigm combined with EEG measurements (Chapter III). Subsequently, the impact of age was tested using the paradigm mentioned above (Chapter IV). The final study investigated whether the cholinergic muscarinic type 1 receptor antagonist biperiden (BIP) can be used to model age-related memory impairment pharmacologically (Chapter $V$ ). In the present chapter, the 
overall findings of the studies presented in this thesis are summarized and discussed. Lastly, some directions for future research are highlighted.

\section{VI.1. Overview of the main Findings}

Chapter II showed that new item identification is superior to old item recognition when pre-experimentally familiar and pre-experimentally unfamiliar materials are shallowly encoded, but not when they are first encoded deeply and then re-encoded. Thus, such findings indicate that stimulus novelty (i.e., no existing memory representation) can provide processing benefits when contrasted with items relying on weak memory. However, when old item memory is strongly embedded, this processing advantage dissipates. In Chapter III, it was found that successful new item identification was marked by a combination of the absence of familiarity (N400) and recollection (P600) for the studied figures. For both the abstract figures and the non-words, the parietal P600 was found to differentiate between the old and new items (late old/new effects). In Chapter IV, it was found that older adults relative to the young showed an impairment in the correct identification of new items. As indicated by the lower discriminability indexes, the elderly also had difficulties discriminating the strongly (drawn) and the weakly (studied) embedded abstract figures but not the non-words. Age-related differences in speed of processing were also only evident with the abstract figures. Additionally, it was found that discrimination performance was affected by memory strength in the young and the elderly. The presented findings agree with previous research on age-related impairment in new item recognition, which can be attributed to misrecollection, decreased sensitivity to novelty, and less accurate novelty assessment in the elderly than the young. The detected age effects on the discriminability of the drawn and studied abstract figures agree with the age-related impairment in the perceptual 
encoding hypothesis and support the notion related to the need for environmental support to reduce age effects. Thus, the results further support the notion that recognition performance in aging is only impaired under certain conditions and extend the understanding of the mechanisms underlying agerelated changes on the memory of pre-experimentally unfamiliar items. Chapter $V$ showed that BIP impaired the correct recognition and prolonged the abstract figures' reaction times. Interestingly, BIP only impaired the recognition of the drawn (deeply memorized and repeated items relying on strong memory) and studied (shallowly memorized and not repeated items relying on weak memory) figures but not the correct identification of the new abstract figures. Furthermore, the processing of the non-words was not affected by BIP treatment. These druginduced effects were, to a great extent, the opposite of the pattern seen in healthy aging. Therefore, BIP cannot be considered a valid model of healthy aging for this old/new recognition memory paradigm. The results of these experiments extend our understanding of the mechanisms underlying the processing of preexperimentally unfamiliar stimuli and age-related changes during processing.

\section{VI.2. The current Paradigm in light of well-known Memory Phenomena and Aging}

In line with previous research involving pre-experimentally familiar materials, the paradigm has reliably and consistently shown throughout all presented experiments the benefits of deeper Levels of Processing (LOP) and repetition over shallow encoding without repetition (Craik \& Lockhart, 1972; Craik \& Tulving, 1975; Hintzman \& Curran, 1997; Newell \& Andrews, 2004). Furthermore, such findings were independent of pre-experimental familiarity (see Chapter II), age (see Chapter IV), and the pharmacological treatment with BIP (see Chapter V). 
As previously described, deeper LOP and repetition are known to overall improve memory and discrimination performance (Craik \& Lockhart, 1972; Craik \& Tulving, 1975; Hintzman \& Curran, 1997; Newell \& Andrews, 2004). Indeed, the results presented in this dissertation were consistent. Thus, deep encoding (drawing and semantic processing) and repetition enhanced recognition accuracy and induced faster reaction times (RT) compared to shallow encoding (studying) when the visual and verbal items were pre-experimentally familiar (see Chapter II) and pre-experimentally unfamiliar in the young (see Chapter II and III) and the elderly (see Chapter IV) and under the influence of BIP (see Chapter V). Accordingly, it was also easier to discriminate the drawn/semantically processed than the studied items. These factors denote stronger memories (Marzi \& Viggiano, 2010; Wixted \& Stretch, 2004; Yonelinas, 2002). In contrast, the shallowly encoded items resulted in lower discriminability index scores. These factors designate weaker memories (Hirshman, 1995).

The EEG findings indicated differences in the processing of the abstract figures and non-words during recognition (see Chapter III). In agreement with the literature, the studied abstract figures compared to the drawn evoked more negative familiarity-based N400 and less positive recollection-based P600 effects (Friedman \& Johnson, 2000; Marzi \& Viggiano, 2010; Rugg \& Allan, 2000). However, the processing of the non-words was different. Namely, instead of affecting the N400 and P600 components, the processing of the semantically processed and studied non-words showed larger amplitudes and prolonged latencies only concerning the P600 component. The lack of effects on the early N400 substantiates that recognition of the non-words does not rely on semantic processing (Brouwer, Fitz, \& Hoeks, 2012; MacKenzie \& Donaldson, 2009; Voss \& Paller, 2009; Wang et al., 2017). Altogether, such findings implicate that while the abstract figures and non-words are processed differently, their recognition likely involves familiarity- and recollection-based processing, similar to 
meaningful figures and words (Gardiner \& Java, 1990; Wixted, 2007; Yonelinas, 2002). However, further studies are required to substantiate this claim.

The data reported in this dissertation showed detectable age differences concerning the discriminability of the drawn and studied visual but not verbal items (see Chapter IV). In agreement with previous research, this was likely due to the elderly making more false alarms than the young participants (Fraundorf, Hourihan, Peters, \& Benjamin, 2019; Norman \& Schacter, 1997). Thus, our findings can be reconciled with the environmental support hypothesis (Craik, 1986) and the age-related impairment in the perceptual encoding hypothesis (Trahan, Larrabee, \& Levin, 1986). However, they cannot be explained by ceiling effects (Uttl, Henry, \& Baltimore, 2007), or the processing theory account of cognitive aging (Craik, 2002).

It has been proposed that ceiling effects for young participants (i.g., too easy tasks ) can mask any age-related differences in recognition memory (Uttl, Henry, \& Baltimore, 2007). However, it seems unlikely that the tasks in the current paradigm were too easy for the young. Namely, the stimuli were preexperimentally unfamiliar, the interstimulus interval (ISI) was relatively short, and the recognition phase involved 90 items. Also, the studied items' hit rates were low, and the miss rates were high, indicating that the task should not have been easy for the young. Furthermore, there was no detectable age difference concerning the hit rates. Thus, our findings corroborate those of Danckert and Craik (2013). Danckert and Craik (2013) attempted to control for ceiling effects when testing the recognition memory of the elderly relative to the young. They used a paradigm similar to ours, involving a deep encoding task (pleasantness ratings) and a shallow memorization task during learning. In addition, they controlled for ceiling effects by applying recall and recognition tests in close succession, matching high and low-performing young and elderly participants, and using a long item set in their Experiment 1 and a shorter one in Experiment 
2. The outcome indicated that while age differences were more considerable on the recall task, there were no ceiling effects on the recognition test. Thus, the authors concluded that ceiling effects could not account for age-related differences in recognition memory.

If the processing theory account of cognitive aging applied to abstract figures and non-words, there should have been no age effects found for the discriminability for the drawn and semantically processed items (Craik, 2002). Yet, age effects were apparent for the drawn items despite their mnemonic processing. Interestingly and in agreement with previous research using similar stimuli, age affected the discriminability of both types of abstract figures but not the non-words (Badham \& Maylor, 2011; Harker \& Riege, 1985; Smith, Park, Cherry, \& Berkovsky, 1990). Thus, the environmental support hypothesis seems to provide a more plausible explanation (Craik, 1986). In other words, the complexity of the simple line drawings in the present paradigm likely could not provide sufficient perceptual support that could have compensated the memory of the elderly (Harker \& Riege, 1985; Smith, Park, Cherry, \& Berkovsky, 1990). In contrast, concerning the non-words, it seems likely that the age-related impairment was absent since the rhyming words provided sufficient environmental support. Similar findings were reported by Craik and McDowd (1987). Thus, environmental support seems to be more appropriate than mnemonic instructions for reducing age effects. However, this notion needs further investigation.

\section{VI.3. The Impact of Memory Strength and Age on the Recognition Performance}

The behavioral results of the experiments reported in this dissertation have consistently shown that memory strength affects recognition performance. This finding was independent of pre-experimental familiarity and age. Namely, 
the recognition of the deeply encoded and repeated (drawn) figures was comparable or even exceeded the correct identification of the new ones. Accordingly, as anticipated and in line with previous research (Delhaye, Bastin, Moulin, Besson, \& Barbeau, 2017; Kafkas \& Montaldi, 2014; Kafkas \& Montaldi, 2018), reactions were faster to the drawn than the new figures. In contrast, it has been found that correct new item identification was superior to recognition memory when pre-experimentally unfamiliar stimuli were shallowly encoded. The results were slightly different for the verbal items since the semantically processed stimuli were recognized as 'old' just as accurately as the new ones were identified as 'new'. The visual and verbal items' old/new recognition performance was likely different because of the differences in LOP of the deep versus the intermediate encoding task used with abstract figures and non-words, respectively. Previous research has demonstrated that drawing compared to writing (Wammes, Meade, \& Fernandes, 2016) or phonological encoding reaches deeper during processing and establishes stronger memories (Matlin, 2005). Consequently, the drawing task must have increased the experimentally induced familiarity of the figures to a greater extent than the rhyming word task could achieve with the verbal stimuli.

Accordingly, the electrophysiological data of the abstract figures and non-words showed the late old/new effect in that the processing of the drawn stimuli compared to the new ones evoked larger P600 amplitudes and prompted longer latency. The parietal late old/new effect entails larger amplitudes for the pre-exposed than not pre-exposed items arising from the involvement of recollection type memory (Curran \& Hancock, 2007; Friedman \& Johnson, 2000; Otten, Sveen, \& Quayle, 2007; Rugg \& Curran, 2007; Ziegler, Besson, Jacobs, \& Nazir, 1995). The P600's modulation is thought to index the reactivation and reanalysis of existing memory representations, including the stimulus and its contextual information (Friedman \& Johnson, 2000; Rugg \& Curran, 2007; 
Yonelinas, 2002). Thus, the current findings show that the drawn figures and the semantically processed non-words were well-recognized and could be correctly discriminated against the new items.

For both the visual and verbal items, new item identification was consistently more accurate than the correct recognition of items relying on weak memory representations (studied items). As such, the current data show that it was likely more difficult to discriminate the shallowly encoded items from the new ones correctly. In other words, the studied stimuli were likely perceived as more 'new' than 'old'. Accordingly, the RT-s of the studied and new stimuli were not significantly different. This differential effect of deep and shallow LOP and repetition on the recognition performance indicates a clear effect of memory strength. Prior research on memory performance involving pre-experimentally familiar stimuli has typically shown that stimulus novelty provides processing advantages over familiarity (Donaldson, 1992; Kafkas \& Montaldi, 2014; Kafkas \& Montaldi, 2018; Kumaran \& Maguire, 2007). However, when preexperimentally unfamiliar items are memorized and then their recognition is tested, it seems that experimentally induced familiarity can support discrimination comparably to new item identification. It should be noted that the discrimination performance of the new and familiar items was already at a high level, which could account for the lack of detectable differences. Nevertheless, a ceiling effect seems less likely, as described above.

Improved new item identification of the abstract figures was marked by the early old/new effect (FN400), which, as discussed above, was not detected with the non-words. The frontocentral FN400 is postulated to represent the effective use of familiarity during old/new discrimination (Curran \& Hancock, 2007; Friedman \& Johnson, 2000; Rugg \& Curran, 2007). As such, early old/new effects are seen as more negative amplitudes for new stimuli that were correctly endorsed as 'new' compared to previously seen old stimuli that were recognized 
as 'old' (Curran \& Hancock, 2007; Friedman \& Johnson, 2000; Rugg \& Curran, 2007). Thus, the current findings support the notion that the FN400 reflects familiarity-based discrimination and correct identification of new items (Curran \& Hancock, 2007). Namely, similarly to the results of Rugg and Curran (2007), the FN400 peaks related to the processing of the studied and new figures were comparable over the posterior cluster. However, the peaks related to the processing of the drawn figures were less negative than those of the new, which agrees with the notion that deep encoding facilitates recognition performance (Friedman \& Johnson, 2000).

One of the most prominent discrimination performance theories is the comparator or match/mismatch model (Kumaran \& Maguire, 2007). According to this model, the input undergoes a preliminary evaluation followed by match/mismatch signal initiation. If the stimulus matches an existing representation, it is recognized as familiar. If there is no match or a mismatch is found, novelty is detected. This comparison is assumed to be carried out dually by the hippocampus, a structure known to be crucial for memory and novelty processing (Kafkas \& Montaldi, 2014; Kafkas \& Montaldi, 2018; Kumaran \& Maguire, 2007). Such claims are supported by neuroimaging studies that have identified two separate segments, such as the anterior and posterior hippocampus, that serve different functions during old/new recognition (Kafkas \& Montaldi, 2018; Kumaran \& Maguire, 2006; Poppenk \& Moscovitch, 2011).

For example, Kumaran and Maguire (2006) showed increased activation in the anterior hippocampus in response to novelty. Moreover, Kafkas and Montaldi (2018) proposed that besides active novelty detection, the anterior hippocampus's role is efficient communication of stimulus significance to the striatum and the dopaminergic midbrain, where novelty salience is further appraised. Poppenk and Moscovitch (2011) showed that recollective memory relies on the posterior but not anterior hippocampus in a population of healthy 
young volunteers. Moreover, they proposed that the hippocampus' functional connectivity to neocortical regions and other midbrain structures mediates the posterior hippocampus' role in memory. Thus, if both the anterior and the posterior hippocampal segments and their connections to other cortical areas are functional, stimulus novelty and familiarity can theoretically aid recognition memory with comparable efficacy. Indeed, new item identification in all experiments was comparable to the correct recognition of the deeply processed and repeated materials that, as discussed above, likely involved a solid match to existing templates. Such logic also implies that if the match is weak, new item identification can be more accurate than old item recognition, which was found consistently.

Furthermore, the elderly compared to the young showed a marked decline in correct new item identification. Also, new items prompted an increase in the number of false alarms, which is often seen in older adults (Gallo, Cotel, Moore, \& Schacter, 2007; Kroll, Knight, Metcalfe, Wolf, \& Tulving, 1996). These findings were associated to theories such as the misrecollection account of cognitive aging that is based on false memories (Dodson, Bawa, \& Krueger, 2007), an age-related decreased sensitivity to novelty (Czigler, Pato, Poszet, \& Balazs, 2006), and the age-dependent impairment in novelty assessment (Bastin, Delhaye, Moulin, \& Barbeau, 2019; Daffner et al., 2011; de Chastelaine, Mattson, Wang, Donley, \& Rugg, 2017) (see Chapter IV). Here, the latter is further discussed in alignment with the comparator model (Kumaran \& Maguire, 2006).

The volume and connectivity of the hippocampus are known to deteriorate with age (Blum, Habeck, Steffener, Razlighi, \& Stern, 2014; Bowman \& Dennis, 2015; de Chastelaine, Mattson, Wang, Donley, \& Rugg, 2017; Grady, McIntosh, \& Craik, 2003). However, the anterior hippocampus degenerates before the posterior part in aging (Blum, Habeck, Steffener, Razlighi, \& Stern, 2014). Also, Blum, Habeck, Steffener, Razlighi, and Stern (2014) found an overall 
depreciated functional connectivity of the hippocampus to cortical areas in the elderly compared to the young, but there was a relative increase in posterior hippocampal connectivity. Hence, as suggested by the comparator model and neuroimaging studies, this could account for decreased accuracy performance concerning the correct rejection of the new items. In other words, worse novelty assessment, and thus less accurate new item identification, in the elderly could result from decreased connectivity and a less operational anterior hippocampus. Conversely, the relatively preserved posterior hippocampal function could explain why the memorized pre-experimentally unfamiliar stimuli did not show prominent age effects on the hit rates. This bears future investigation. Nevertheless, the presented findings support the notion that cognitive aging concerns domain-specific insufficiencies rather than a single global cognitive deficit (Benjamin, 2010; Fraundorf, Hourihan, Peters, \& Benjamin, 2019).

\section{VI.4. The pharmacological Model of healthy Aging}

For BIP to be an adequate pharmacological model of healthy aging, the drug should have impaired correct new item rejection while leaving the hits rates of the drawn/semantically processed and studied abstract figures and non-words unaffected. Also, drug effects should have been found for the discriminability indexes $\left(\mathrm{A}^{\prime}\right)$. Neither of these effects were detected. In contrast to our expectations, the studied abstract figures were less well recognized after the drug treatment, and the rest of the mentioned effects were not detected. However, considering that BIP only affected the processing of the abstract figures, it cannot be ruled out that our findings were related to a response bias in the PLA conditions, as discussed in Chapter $\boldsymbol{V}$. However, as anticipated, the drug prolonged the reaction times of the drawn and the studied abstract figures. Thus, even though the BIP-induced effects were different for the abstract figures and 
the non-words, they did not fully model the typical age-related deficits in recognition performance.

BIP impaired the recognition accuracy of the drawn and studied figures, which is in agreement with previous research on BIP's memory impairing effect in healthy volunteers (Sambeth, Riedel, Klinkenberg, Kahkonen, \& Blokland, 2015; Silver \& Geraisy, 1995) but does not comply with the findings on the agerelated impairment in recognition memory performance described in Chapter IV. Therefore, it could be that the M1 receptors and the cholinergic transmission are less affected by healthy cognitive aging than dementia or AD. These findings corroborate those reported by Muller, Stoll, Schubert, and Gelbmann (1991). This review article revealed that, unlike $\mathrm{AD}$, healthy aging does not involve degenerations of the cholinergic innervation of the cortex and the hippocampus but might negatively affect acetylcholine $(\mathrm{ACh})$ release and muscarinic receptor responsiveness. However, the authors argued that age-associated decrements in learning and memory could not be solely attributed to deficits in the cholinergic system's functioning and proposed that other neurotransmitter systems could also play a role. Nevertheless, similarly to healthy aging, BIP increased the processing time of the abstract figures without affecting the non-words, which, however, does not substantiate BIP's ability to model cognitive aging in the present paradigm.

In support of this notion, BIP also did not impair the correct identification of the new items, which is surprising considering that BIP is a cholinergic M1 receptor antagonist (Blokland, Sambeth, Prickaerts, \& Riedel, 2016; Kostelnik, Cegan, \& Pohanka, 2017). In general, new stimuli are assumed to initiate a cascade of neural events, including activation of the cholinergic, noradrenergic, and the dopaminergic systems (Caldenhove, Borghans, Blokland, \& Sambeth, 2017; Kafkas \& Montaldi, 2018; Rangel-Gomez \& Meeter, 2016; Schomaker \& Meeter, 2015; Wittmann, Bunzeck, Dolan, \& Duzel, 2007). Thus, the 
lack of drug effects on new item identification indicates that their discrimination likely involved noradrenergic or dopaminergic neurotransmission rather than cholinergic. Such an idea, while being speculative, since we did not specifically investigate the effects of a norepinephrine (NE) or dopamine (DA) antagonist to test this hypothesis, is nevertheless possible for two reasons.

First, most of the evidence on ACh's involvement in novelty detection stems from odd-ball tasks (Caldenhove, Borghans, Blokland, \& Sambeth, 2017; Kafkas \& Montaldi, 2015; Rangel-Gomez \& Meeter, 2016). In these paradigms, the deviation of an irrelevant or unexpected visual or auditory stimulus yields a preattentive capture of attention reflected by changes in EEG and fMRI (Amenedo \& Diaz, 1998; Friedman \& Simpson, 1994; Parmentier \& Andrés, 2010; Pato \& Czigler, 2011; Weisz \& Czigler, 2006). Accordingly, improved detection of novelty in such paradigms has been attributed to violation of expectations (Vachon, Hughes, \& Jones, 2012). However, detecting a highly deviant stimulus (e.g., a red circle among blue squares) does not thoroughly compare to identifying a new stimulus in an old/new recognition task in which the items are relatively similar and are consistently compared to existing memory templates.

Second, according to evidence from recognition memory tasks, new items can initiate a hippocampal novelty signal mediated by DA. This signal is assumed to activate the substantia nigra/ventral tegmental area and trigger improved functional connectivity of this region to the hippocampus via DA (Kafkas \& Montaldi, 2015; Lisman \& Grace, 2005). Moreover, the anticipation of novelty has been shown to activate the reward system and, specifically, the hippocampus during processing (Bunzeck \& Duzel, 2006; Schomaker \& Meeter, 2015; Wittmann, Bunzeck, Dolan, \& Duzel, 2007). Altogether, such evidence suggests that novelty can provide mnemonic benefits via its reward characteristics, which explains the lack of BIP-induced impairment in new item identification. When combined with the current findings, this research line shows 
that novelty can provide processing benefits overall by stimulating the participants' DA-driven reward system. However, novel stimuli are likely not per se preferentially processed by the memory system, which accommodates familiar information comparably unless it relies on strong memories.

\section{VI.5. Implications and Future Directions}

Altogether, it can be said that the benefits of deeper LOP and repetition over shallow encoding (Craik \& Lockhart, 1972; Craik \& Tulving, 1975; Hintzman \& Curran, 1997; Newell \& Andrews, 2004) apply to pre-experimentally unfamiliar old item recognition. Moreover, it seems that aging affects the ability to discriminate pre-experimentally unfamiliar visual but not verbal items, indicating domain-specific deficits (Fraundorf, Hourihan, Peters, \& Benjamin, 2019), which may be reduced with sufficient environmental support (Craik, 1986) rather than with mnemonic instructions (Bridger et al., 2017; Fraundorf, Hourihan, Peters, \& Benjamin, 2019; Naveh-Benjamin \& Mayr, 2018). Additionally, such deficits cannot be achieved by cholinergic modulation using the current paradigm.

Moreover, as discussed in this dissertation, the models of recognition memory and their neural bases indicate that recognition performance depends on the memory strength in young adults, the elderly, and when cholinergic modulation is used. Electrophysiologically, accurate memory performance of the abstract figures in the current paradigm can be explained by a combination of effective use of familiarity (FN400) and recollection (P600) type memory for items relying on strong memories. In contrast, the poorer performance involving the items relying on weak memory can be explained by a combination of ineffective use of familiarity (FN400) and a lack of recollection type memory (P600). Accordingly, these findings on the early and late old/new effects during the 
processing of the abstract figures point towards dual-route processing (Mandler, 2008; Rugg \& Curran, 2007; Yonelinas, 2002). However, further studies are required in which aspects of recollection and familiarity are directly investigated with EEG and an extended version of the current paradigm.

Furthermore, Kumaran and Maguire (2007) argued that novelty detection plays an essential role in the continuous updating of our representations of the world (i.e., unsupervised learning). Moreover, exploration is proposed to continue unless novel information has been integrated into memory representations (e.g., cognitive map) (O'Keefe \& Nadel, 1978). Based on the presented findings, it seems that new items are preferentially processed when they are contrasted with items having fragile memories. However, the advantage of novelty dissipates when the judgment involves familiar items relying on strong memories. Thus, both novelty and familiarity can support correct recognition comparably unless familiarity relies on strongly embedded memories. Such findings suggest that novelty may not merely be 'not familiar'. Rather, novelty and familiarity might be two independent functions that offer distinct contributions to discrimination decisions that show domain-specific impairments in cognitive aging and likely rely on other than the cholinergic neurotransmitter system considering the involvement of pre-experimentally unfamiliar materials.

To further investigate and support the claims presented in this work, future research could extend the present paradigm with confidence judgments and questionnaires that could reveal which specific strategies the participants used during memorization. An intriguing follow-up study could examine the relation between recognition and recall using an extended version of the current paradigm. It would also be of interest to combine behavioral investigations with other neuroimaging methods to further examine age-related recognition performance. Additional investigations of age-related compensation, executive 
function, and hippocampal activity/connectivity could help understand the nature of the conditions that underly the processing of pre-experimentally unfamiliar items. Moreover, considering the cholinergic modulation's ineffectiveness in mimicking age-related cognitive impairment, it is suggested to investigate the effects of a DA- or a NE-based pharmacological model. Such an approach would enable the establishment of links between the distinct neuromodulatory mechanisms and behavioral effects of novelty and familiarity processing also involved in aging. 
152 


\section{VI.6. References}

Ally, B. A. (2012). Using pictures and words to understand recognition memory deterioration in amnestic mild cognitive impairment and Alzheimer's disease: a review. Curr Neurol Neurosci Rep, 12(6), 687-694. doi:10.1007/s11910-012-0310-7

Ally, B. A., Waring, J. D., Beth, E. H., McKeever, J. D., Milberg, W. P., \& Budson, A. E. (2008). Aging memory for pictures: using high-density eventrelated potentials to understand the effect of aging on the picture superiority effect. Neuropsychologia, 46(2), 679-689. doi:10.1016/j.neuropsychologia.2007.09.011

Amenedo, E., \& Diaz, F. (1998). Aging-related changes in processing of nontarget and target stimuli during an auditory oddball task. Biological Psychology, 48(3), 235-267.

Anderson, J. R. (1983). The architecture of cognition. Hillsdale: NJ: Erlbaum.

Badham, S. P., \& Maylor, E. A. (2011). Age-related associative deficits are absent with nonwords. Psychol Aging, 26(3), 689-694. doi:10.1037/a0022205

Badham, S. P., Poirier, M., Gandhi, N., Hadjivassiliou, A., \& Maylor, E. A. (2016). Aging and memory as discrimination: Influences of encoding specificity, cue overload, and prior knowledge. Psychol Aging, 31(7), 758-770. doi:10.1037/pag0000126

Bastin, C., Delhaye, E., Moulin, C., \& Barbeau, E. J. (2019). Novelty processing and memory impairment in Alzheimer's disease: A review. Neurosci Biobehav Rev, 100, 237-249. doi:10.1016/j.neubiorev.2019.02.021

Beisteiner, R., Huter, D., Edward, V., Koch, G., Franzen, P., Egkher, A., . . Lang, W. (1996). Brain potentials with old/new distinction of non-words and geometric figures. Electroencephalography and Clinical Neurophysiology, 99, 517-526

Belleville, S., Menard, M. C., \& Lepage, E. (2011). Impact of novelty and type of material on recognition in healthy older adults and persons with mild cognitive impairment. Neuropsychologia, 49(10), 2856-2865.

doi:10.1016/j.neuropsychologia.2011.06.011

Bellhouse-King, M. W., \& Standing, L. G. (2007). Recognition memory for concrete, regular abstract, and diverse abstract pictures. Perceptual and Motor Skills, 104, 758-762.

Benjamin, A. S. (2010). Representational explanations of "process" dissociations in recognition: the DRYAD theory of aging and memory judgments. Psychol Rev, 117(4), 1055-1079. doi:10.1037/a0020810

Benjamin, A. S., \& Bawa, S. (2004). Distractor plausibility and criterion placement in recognition. Journal of Memory and Language, 51(2), 159172. doi:10.1016/j.jml.2004.04.001 
Benjamin, A. S., Diaz, M., \& Wee, S. (2009). Signal detection with criterion noise: applications to recognition memory. Psychol Rev, 116(1), 84-115. doi:10.1037/a0014351

Blokland, A., Sambeth, A., Prickaerts, J., \& Riedel, W. J. (2016). Why an M1 Antagonist Could Be a More Selective Model for Memory Impairment than Scopolamine. Front Neurol, 7, 167. doi:10.3389/fneur.2016.00167

Blum, S., Habeck, C., Steffener, J., Razlighi, Q., \& Stern, Y. (2014). Functional connectivity of the posterior hippocampus is more dominant as we age. Cogn Neurosci, 5(3-4), 150-159. doi:10.1080/17588928.2014.975680

Borghans, L., Sambeth, A., \& Blokland, A. (2020). Biperiden Selectively Impairs Verbal Episodic Memory in a Dose- and Time-Dependent Manner in Healthy Subjects. J Clin Psychopharmacol, 40(1), 30-37. doi:10.1097/JCP.0000000000001157

Borghans, L. G., Blokland, A., \& Sambeth, A. (2017). Effects of biperiden and acute tryptophan depletion and their combination on verbal word memory and EEG. Psychopharmacology (Berl), 234(7), 1135-1143. doi:10.1007/s00213-017-4549-1

Borghans, L. G., Sambeth, A., Prickaerts, J., Ramaekers, J. G., \& Blokland, A. (2018). The effects of the soluble guanylate cyclase stimulator riociguat on memory performance in healthy volunteers with a biperideninduced memory impairment. Psychopharmacology, 235(8), 2407-2416.

Boucher, O., Chouinard-Leclaire, C., Muckle, G., Westerlund, A., Burden, M. J., Jacobson, S. W., \& Jacobson, J. L. (2016). An ERP study of recognition memory for concrete and abstract pictures in school-aged children. Int J Psychophysiol, 106, 106-114. doi:10.1016/j.ijpsycho.2016.06.009

Bowman, C. R., \& Dennis, N. A. (2015). Age differences in the neural correlates of novelty processing: The effects of item-relatedness. Brain Research, 1612, 2-15. doi:10.1016/j.brainres.2014.08.006

Bridger, E. K., Kursawe, A. L., Bader, R., Tibon, R., Gronau, N., Levy, D. A., \& Mecklinger, A. (2017). Age effects on associative memory for novel picture pairings. Brain Res, 1664, 102-115. doi:10.1016/j.brainres.2017.03.031

Brouwer, H., Fitz, H., \& Hoeks, J. (2012). Getting real about semantic illusions: rethinking the functional role of the P600 in language comprehension. Brain Res, 1446, 127-143. doi:10.1016/j.brainres.2012.01.055

Brown, S., \& Steyvers, M. (2005). The dynamics of experimentally induced criterion shifts. J Exp Psychol Learn Mem Cogn, 31(4), 587-599. doi:10.1037/0278-7393.31.4.587

Bunzeck, N., \& Duzel, E. (2006). Absolute coding of stimulus novelty in the human substantia nigra/VTA. Neuron, 51(3), 369-379. doi:10.1016/j.neuron.2006.06.021 
Caldenhove, S., Borghans, L. G. J. M., Blokland, A., \& Sambeth, A. (2017). Role of acetylcholine and serotonin in novelty processing using an oddball paradigm. Behavioural Brain Research, 331, 199-204. doi:10.1016/j.bbr.2017.05.031

Cherry, K. E., Hawley, K. S., Jackson, E. M., Volaufova, J., Su, L. J., \& Jazwinski, S. M. (2008). Pictorial superiority effects in oldest-old adults. Memory, 16(7), 728-741. doi:10.1080/09658210802215534

Craik, F. I. (1986). A functional account of age differences in memory. In F. Klix $\mathcal{E} H$. Hagendorf (Eds.), Human memory and cognitive capabilities: Mechanisms and performances. North Holland, the Netherlands: Elsevier.

Craik, F. I. (2002). Levels of processing: past, present and future? Memory, 10(56), 305-318. doi:10.1080/09658210244000135

Craik, F. I., \& Byrd, M. (1982). Aging and cognitive deficits: The role of attentional resources. In F. I. Craik $\mathcal{E} S$. Trehub (Eds.), Aging and cognitive processes. New York: NY- Plenum.

Craik, F. I., \& Lockhart, R. S. (1972). Levels of processing: A framework for memory research. Journal of Verbal Learning and Verbal Behaviour, 11, 671-684.

Craik, F. I., \& McDowd, J. M. (1987). Age differences in recall and recognition. Journal of Experimental Psychology: Learning, Memory, and Cognition, 13(3), 474-479. doi:10.1037/0278-7393.13.3.474

Craik, F. I., \& Rose, N. S. (2012). Memory encoding and aging: a neurocognitive perspective. Neurosci Biobehav Rev, 36(7), 1729-1739. doi:10.1016/j.neubiorev.2011.11.007

Craik, F. I., \& Tulving, E. (1975). Depth of processing and the retention of words in episodic memory. Journal of Experimental Psychology: General, 104, 268-294.

Curran, T., \& Hancock, J. (2007). The FN400 indexes familiarity-based recognition of faces. Neuroimage, 36(2), 464-471. doi:10.1016/j.neuroimage.2006.12.016

Cycowicz, Y. M., \& Friedman, D. (2007). Visual novel stimuli in an ERP novelty oddball paradigm: effects of familiarity on repetition and recognition memory. Psychophysiology, 44(1), 11-29. doi:10.1111/j.14698986.2006.00481.x

Czigler, I., Pato, L., Poszet, E., \& Balazs, L. (2006). Age and novelty: eventrelated potentials to visual stimuli within an auditory oddball--visual detection task. Int J Psychophysiol, 62(2), 290-299. doi:10.1016/j.ijpsycho.2006.05.008

Daffner, K. R., Mesulam, M. M., Scinto, L. F., Calvo, V., Faust, R., \& Holcomb, P. J. (2000). An electrophysiological index of stimulus unfamiliarity. Psychophysiology 37, 737-747. 
Daffner, K. R., Ryan, K. K., Williams, D. M., Budson, A. E., Rentz, D. M., Wolk, D. A., \& Holcomb, P. J. (2006). Increased responsiveness to novelty is associated with successful cognitive aging.J Cog Neurosci, 2006.pdf > . Journal of Cognitive Neuroscience, 18(10), 1759-1773.

Daffner, K. R., Scinto, L. F. M., Weitzman, A. M., Faust, R., Rentz, D. M., \& Budson, A. E. (2003). Frontal and parietal components of a cerebral network mediating voluntary attention to novel events. Journal of Cognitive Neuroscience 15(2), 294-313.

Daffner, K. R., Sun, X., Tarbi, E. C., Rentz, D. M., Holcomb, P. J., \& Riis, J. L. (2011). Does compensatory neural activity survive old-old age? Neuroimage, 54(1), 427-438. doi:10.1016/j.neuroimage.2010.08.006

Dalla Barba, G. (1997). Recognition Memory and Recollective Experience in Alzheimer's Disease. Memory, 5(6), 657-672.

Danckert, S. L., \& Craik, F. I. (2013). Does aging affect recall more than recognition memory? Psychol Aging, 28(4), 902-909. doi:10.1037/a0033263

de Chastelaine, M., Mattson, J. T., Wang, T. H., Donley, B. E., \& Rugg, M. D. (2017). Independent contributions of fMRI familiarity and novelty effects to recognition memory and their stability across the adult lifespan. Neuroimage, 156, 340-351. doi:10.1016/j.neuroimage.2017.05.039

Delhaye, E., Bastin, C., Moulin, C. J. A., Besson, G., \& Barbeau, E. J. (2017). Bridging novelty and familiarity-based recognition memory: A matter of timing. Visual Cognition, 25(9-10), 949-955. doi:10.1080/13506285.2017.1362090

Dijkstra, K., Farquhar, J., \& Desain, P. (2019). Electrophysiological responses of relatedness to consecutive word stimuli in relation to an actively recollected target word. Sci Rep, 9(1), 14514. doi:10.1038/s41598-01951011-4

Dobbins, I. G., Kroll, N. E. A., Yonelinas, A. P., \& Liu, Q. (1998). Distinctiveness in Recognition and Free Recall: The Role of Recollection in the Rejection of the Familiar. Journal of Memory and Language, 38, 381-400.

Dodson, C. S., Bawa, S., \& Krueger, L. E. (2007). Aging, metamemory, and highconfidence errors: a misrecollection account. Psychol Aging, 22(1), 122133. doi:10.1037/0882-7974.22.1.122

Donaldson, W. (1992). Measuring Recognition Memory. Journal of Experimental Psychology: General, 121(3), 275-277.

Dopkins, S., Sargent, J., \& Ngo, C. T. (2010). The bias for a recognition judgement depends on the response emitted in a prior recognition judgement. Memory, 18(3), 272-283. 
Ekuni, R., Vaz, L. J., \& Bueno, O. F. A. (2011). Levels of processing: the evolution of a framework. Psychology and Neuroscience, 4(3), 333-339. doi:10.3922/j.psns.2011.3.006

Estes, W. K. (2002). Traps in the route to models of memory and decision. Psychonomic Bulletin and Review, 9, 3-25.

Estes, W. K., \& Maddox, W. T. (1995). Interactions of stimulus attributes, base rates, and feedback in recognition Journal of Experimental Psychology: Learning, Memory, and Cognition, 31, 1075-1095.

Eysenck, M. W. (1978a). Levels of processing: A critique. British Journal of Psychology, 69, 157-169.

Eysenck, M. W. (1978b). Levels of processing: A reply to Lockhart and Craik. British Journal of Psychology, 69, 177-178.

Fandakova, Y., Lindenberger, U., \& Shing, Y. L. (2014). Deficits in processspecific prefrontal and hippocampal activations contribute to adult age differences in episodic memory interference. Cereb Cortex, 24(7), 18321844. doi:10.1093/cercor/bht034

Faul, F., Erdfelder, E., Lang, A. G., \& Buchner, A. (2007). G*Power 3: a flexible statistical power analysis program for the social, behavioral, and biomedical sciences. Behav Res Methods, 39(2), 175-191.

Ferrari, V., Bradley, M. M., Codispoti, M., \& Lang, P. J. (2010). Detecting novelty and significance.pdf $>$. Journal of Cognitive Neuroscience, 22(2), 404-411.

Finnigan, S., Humphreys, M. S., Dennis, S., \& Geffen, G. (2002). ERP ‘old/new' effects: memory strength and decisional factor(s). Neuropsychologica, 40, 2288-2304.

Fjell, A. M., Walhovd, L. B., \& Reinvang, I. (2005). Age-Differences in Verbal Recognition Memory Revealed by ERP. Clinical EEG and Neuroscience, 36(3), 176-187.

Folstein, M. F., Folstein, S. E., \& McHugh, P. R. (1975). Mini-mental state: a practical method for grading the cognitive state of patients for the clinician. Journal of psychiatric research, 12(3), 189-198.

Fraundorf, S. H., Hourihan, K. L., Peters, R. A., \& Benjamin, A. S. (2019). Aging and recognition memory: A meta-analysis. Psychol Bull, 145(4), 339-371. doi:10.1037/bul0000185

Friedman, D., \& Johnson, J. R. (2000). Event-Related Potential (ERP) Studies of Memory Encoding and Retrieval: A Selective Review. Microscopy Reasearch and Technique 51(6), 6-28.

Friedman, D., \& Simpson, G. V. (1994). ERP amplitude and scal $p$ distribution to target and novel events: effects of temporal order in young, middleaged and older adults.pdf $>$. Cognitive Brain Research, 2, 49-63.

Froger, C., Taconnat, L., Landre, L., Beigneux, K., \& Isingrini, M. (2009). Effects of level of processing at encoding and types of retrieval task in mild 
cognitive impairment and normal aging. J Clin Exp Neuropsychol, 31(3), 312-321. doi:10.1080/13803390802112554

Gallo, D. A., Cotel, S. C., Moore, C. D., \& Schacter, D. L. (2007). Aging can spare recollection-based retrieval monitoring: the importance of event distinctiveness. Psychol Aging, 22(1), 209-213. doi:10.1037/08827974.22.1.209

Gardiner, J. M. (1988). Functional aspects of recollective experience. Memory and Cognition, 16, 309-313.

Gardiner, J. M., \& Java, R. I. (1990). Recollective experience in word and nonword recognition Memory and Cognition, 18(23), 23-30.

Glosser, G., Friedman, R. B., Grugan, P. K., Lee, J. H., \& Grossman, M. (1998). Lexical semantic and associative priming in Alzheimer's disease. Neuropsychology, 12(2), 218-224.

Grady, C. L., McIntosh, A. R., \& Craik, F. I. (2003). Age-related differences in the functional connectivity of the hippocampus during memory encoding. Hippocampus, 13(5), 572-586. doi:10.1002/hipo.10114

Habib, R., McIntosh, A. R., Wheeler, M. A., \& Tulving, E. (2003). Memory encoding and hippocampally-based novelty/familiarity discrimination networks. Neuropsychologia, 41, 271-279.

Harker, J. O., \& Riege, W. H. (1985). Aging and delay effects on recognition of words and designs. Journal of Gerontology 40, 601-604.

Harris, J. D., Cutmore, T. R. H., O'Gorman, J., Finnigan, S., \& Shum, D. H. K. (2013). Electrophysiological Correlates of Perceptual Auditory Priming Without Explicit Recognition Memory. Journal of Psychophysiology, 27(4), 185-195. doi:10.1027/0269-8803/a000104

Hasher, L., Zacks, R., \& May, C. (1999). Inhibitory control, circadian arousal and age (Vol. XVII ). Cambridge: MA- MIT Press.

Hasselmo, M. E. (2006). The role of acetylcholine in learning and memory. Curr Opin Neurobiol, 16(6), 710-715. doi:10.1016/j.conb.2006.09.002

Hasselmo, M. E., Wyble, B. P., \& Wallenstein, G. V. (1996). Encoding and Retrieval of Episodic Memories: Role of Cholinergic and GABAergic Modulation in the Hippocampus. Hippocampus 6, 693-708

Hintzman, D. L., Caulton, D. A., \& Curran, T. (1999). Retrieval constraints and the mirror effect Journal of Experimental Psychology: Learning, Memory and Cognition 20, 275-289.

Hintzman, D. L., \& Curran, T. (1997). Comparing retrieval dynamics in recognition memory and lexical decision. Journal of Experimental Psychology: General, 126, 228-247.

Hirshman, E. (1995). Decision processes in recognition memory: Criterion shifts and the list-strength paradigm. Journal of Experimental Psychology: General Learning, Memory, E Cognition, 21, 302-313. 
Horn, M., Jardri, R., D'Hondt, F., Vaiva, G., Thomas, P., \& Pins, D. (2016). The multiple neural networks of familiarity: A meta-analysis of functional imaging studies. Cogn Affect Behav Neurosci, 16(1), 176-190. doi:10.3758/s13415-015-0392-1

Huang, Y., \& Ferreira, F. (2020). The Application of Signal Detection Theory to Acceptability Judgments. Front Psychol, 11, 73. doi:10.3389/fpsyg.2020.00073

Hulstijn, H. J. (1997). Mnemonic methods in foreign language vocabulary learning- Theoretical considerations and pedagogical implications. In J. Coady \& T. Huckin (Eds.), Second language vocabulary acquisition (pp. 203-224): Cambridge University Press.

Izawa, C. (1980). Proactive versus Retroactive Interference in Recogntion Memory. The Journal of General Psychology, 102, 53-73.

Jones-Gotman, M. (1986). Right hippocampal excision impairs learning and recall of a list of abstract designs. Neuropsychologia, 24(5), 659-670.

Kafkas, A., \& Montaldi, D. (2014). Two separate, but interacting neural systems for familiarity and novelty detection: A dual-route mechanism. Hippocampus, 24(5), 516-527. doi:10.1002/hipo.22241

Kafkas, A., \& Montaldi, D. (2015). Striatal and midbrain connectivity with the hippocampus selectively boosts memory for contextual novelty. Hippocampus, 25(11), 1262-1273. doi:10.1002/hipo.22434

Kafkas, A., \& Montaldi, D. (2018). How do memory systems detect and respond to novelty? Neuroscience Letters, 680, 60-68. doi:10.1016/j.neulet.2018.01.053

Kausler, D. H. (1970). Retention-forgetting as a nomological network for developmental research. In L. R. Goulet E P. B. Baltes (Eds.), Life-span developmental psychology Research and theory. New York: NY Academic Press.

Khateb, A., Pegna, A. J., Michel, C. M., Landis, T., \& Annoni, J.-M. (2002). Dynamics of brain activation during an explicit word and image recognition task: an electrophysiological study. Brain Topography 14, 97213.

Kim, K., \& Glanzer, M. (1993). Speed versus accuracy instructions, study time, and the mirror effect. Journal of Experimental Psychology: Learning, Memory, and Cognition, 19(3), 638-652.

Kim, K. H., Yoon, H. W., \& Park, H. W. (2004). Spatiotemporal brain activation pattern during word/picture perception by native Koreans. Cognitive Neuroscience and Neuropsychology, 15(7), 1099-1103. doi:10.1097/01.wnr.0000126623.64967.38 
Kormi-Nouri, R., Nilsson, L. G., \& Ohta, N. (2005). The novelty effect: support for the Novelty-Encoding Hypothesis. Scandinavian Journal of Psychology, 46(2), 133-143. doi:10.1111/j.1467-9450.2005.00443.x

Kostelnik, A., Cegan, A., \& Pohanka, M. (2017). Anti-Parkinson Drug Biperiden Inhibits Enzyme Acetylcholinesterase. Biomed Res Int, 2017, 2532764. doi:10.1155/2017/2532764

Koutstaal, L., Reddy, C., Jackson, E. M., Prince, S., Cendan, D. L., \& Schachter, D. (2003). False recognition of abstract versus common objects in older and younger adults: testing the semantic categorization account. Journal of Experimental Psychology: Leaning, Memory and Cognition 29, 455-510.

Koutstaal, W., Reddy, C., Jackson, E. M., Prince, S., Cendan, D. L., \& Schacter, D. L. (2003). False recognition of abstract versus common objects in older and younger adults: Testing the semantic categorization account. . Journal of Experimental Psychology: Learning, Memory, and Cognition, 29, 499-510.

Kroll, N. E. A., Knight, R. T., Metcalfe, J., Wolf, E. S., \& Tulving, E. (1996). Cohesion failure as a source of memory illusions. Journal of Memory and Language, 35, 176-196.

Kumaran, D., \& Maguire, E. A. (2006). An unexpected sequence of events: mismatch detection in the human hippocampus. PLoS Biol, 4(12), e424. doi:10.1371/journal.pbio.0040424

Kumaran, D., \& Maguire, E. A. (2007). Which computational mechanisms operate in the hippocampus during novelty detection? Hippocampus, 17(9), 735-748. doi:10.1002/hipo

Lamont, A. C., Stewart-Williams, S., \& Podd, J. L. (2005). Face recognition and aging: Effects of target age and memory load Memory and Cognition, 33(6), 1017-1024.

Levin, H. S., Eisenberg, H. M., \& Benton, A. L. E. (1992). Frontal lobe function and dysfunction. London: Oxford University Press.

Lisman, J. E., \& Grace, A. A. (2005). The hippocampal-VTA loop: controlling the entry of information into long-term memory. Neuron, 46(5), 703-713. doi:10.1016/j.neuron.2005.05.002

Lockhart, R. S., \& Craik, F. I. M. (1978). Levels of processing: A reply to Eysenck. British Journal of Psychology, 69(2), 171-175. doi:10.1111/j.20448295.1978.tb01644.x

Logan, J. M., Sanders, A. L., Snyder, A. Z., Morris, J. C., \& Buckner, R. L. (2002). Under-recruitment and nonselective recruitment: dissociable neural mechanisms associated with aging. Neuron, 33, 827-840.

Luck, S. J. (2005). An Introduction to the Event-Related Potential Technique: Cambridge: MIT. 
MacKenzie, G., \& Donaldson, D. I. (2009). Examining the neural basis of episodic memory: ERP evidence that faces are recollected differently from names. Neuropsychologia, 47(13), 2756-2765.

doi:10.1016/j.neuropsychologia.2009.05.025

Malmberg, K. J. (2008). Recognition memory: a review of the critical findings and an integrated theory for relating them. Cogn Psychol, 57(4), 335-384. doi:10.1016/j.cogpsych.2008.02.004

Mandler, G. (2008). Familiarity Breeds Attempts: A Critical Review of DualProcess Theories of Recognition. Perspectives on Psychological Science, 3(5), 390-399.

Marcel, S., \& Milla'n, J. d. R. (2007). Person authentication using brainwaves (eeg) and maximum a posteriori model adaptation. IEEE Transactions on Pattern Analysis and Machine Intelligence, 29, 743-752.

Marzi, T., \& Viggiano, M. P. (2010). Deep and shallow encoding effects on face recognition: an ERP study. Int J Psychophysiol, 78(3), 239-250. doi:10.1016/j.ijpsycho.2010.08.005

Matlin, M. W. (2005). Cognition (6th ed). Hoboken, Chapter 5 \& Chapter 6 (6 ed. Vol. 6th ed). New Jersey: Wiley.

Muller, W. E., Stoll, L., Schubert, T., \& Gelbmann, C. M. (1991). Central cholinergic functioning and aging. Acta Psychiatr Scand Suppl, 366, 3439. doi:10.1111/j.1600-0447.1991.tb03107.x

Naveh-Benjamin, M., \& Mayr, U. (2018). Age-related differences in associative memory: Empirical evidence and theoretical perspectives. Psychol Aging, 33(1), 1-6. doi:10.1037/pag0000235

Newell, B. R., \& Andrews, S. (2004). Levels of processing effects on implicit and explicit memory tasks: using question position to investigate the lexical-processing hypothesis. Experimental Psychology, 51(2), 132-144. doi:10.1027/1618-3169.51.2.132

Nilsson, L. G. (2003). Memory function in normal aging. Acta Neurologica Scandinavia 7-13.

Noldy, N. E., Stelmack, R. M., \& Campbell, K. B. (1990). Event-related potentials and recognition memory for pictures and words: The effects of intentional and incidental learning. Psychophysiology, 27(4), 417-428.

Norman, K. A., \& Schacter, D. L. (1997). False recognition in younger and older adults: Exploring the characteristics of illusory memories. Memory and Cognition, 25, 838-848.

Norman, K. A., Tepe, K., Nyhus, E., \& Curran, T. (2008). Event-related potential correlates of interference effects on recognition memory. Psychon Bull Rev, 15(1), 36-43. doi:10.3758/pbr.15.1.36

Nyberg, L. (2002). Levels of processing: a view from functional brain imaging. Memory, 10(5-6), 345-348. doi:10.1080/09658210244000171 
Nyberg, L. (2005). Any novelty in hippocampal formation and memory? Curr Opin Neurol, 18(4), 424-428.

Nyhus, E., \& Curran, T. (2009). Semantic and perceptual effects on recognition memory: evidence from ERP. Brain Res, 1283, 102-114. doi:10.1016/j.brainres.2009.05.091

O'Hare, L., \& Goodwin, P. (2018). ERP responses to images of abstract artworks, photographs of natural scenes, and artificially created uncomfortable images. Journal of Cognitive Psychology, 30(5-6), 627-641. doi:10.1080/20445911.2018.1499657

O'Keefe, J., \& Nadel, L. (1978). The Hippocampus as a Cognitive Map. Oxford: Oxford University Press.

Osborne, J. (2002). Notes on the use of data transformations. Practical Assessment8(6). Retrieved from https://scholarworks.umass.edu/cgi/viewcontent.cgi?article=1115\&cont ext=pare website: doi:10.7275/4vng-5608

Otten, L. J., Sveen, J., \& Quayle, A. H. (2007). Distinct Patterns of Neural Activity during Memory Formation of Nonwords versus Words. Journal of Cognitive Neuroscience, 19(11), 1776-1789. doi:10.1162/jocn.2007.19.11.1776

Paivio, A. (1971). Imagery and verbal processes. New York: Holt, Rinehart \& Winston.

Paivio, A. (2007). Mind and its evolution: A dual coding theoretical approach. Mahwah, NJ: Erlbaum.

Paivio, A., \& Desrochers, A. (1981). Mnemonic Techniques in Second-Language Learning. Journal of Educational Psychology, 73(6), 780-795.

Park, D. C., \& Festini, S. B. (2017). Theories of Memory and Aging: A Look at the Past and a Glimpse of the Future. J Gerontol B Psychol Sci Soc Sci, 72(1), 82-90. doi:10.1093/geronb/gbw066

Parmentier, F. B. R., \& Andrés, P. (2010). The Involuntary Capture of Attention by Sound. Experimental Psychology, 57(1), 68-76. doi:10.1027/16183169/a000009

Paszkiel, S. (2017). Characteristics of Question of Blind Source Separation Using Moore-Penrose Pseudoinversion for Reconstruction of EEG Signal. In Automation 2017 (R. Szewczyk, C. Zieliński, \& M. Kaliczyńska Eds. Vol. 550). Cham, Switzerland: Springer International Publishing.

Paszkiel, S. (2020). Using the Raspberry PI2 module and the brain-computer technology for controlling a mobile vehicle. In Automation 2019 (R. Szewczyk, C. Zielin'ski, \& M. Kaliczyn'ska Eds. Vol. 920). Cham, Switzerland: Springer International Publishing. 
Paszkiel, S., \& Szpulak, P. (2018). Methods of Acquisition, Archiving and Biomedical Data Analysis of Brain Functioning. In Proceedings of the 3rd International Scientific Conference on Brain-Computer Interfaces. Opole, Poland: 13-14

Patel, S. H., \& Azzam, P. N. (2005). Characterization of N200 and P300: Selected Studies of the Event-Related Potential. International Journal of Medical Sciences, 2(4), 147-154.

Pato, L., \& Czigler, I. (2011). Effects of novelty on event-related potentials: aging and stimulus replacement. Gerontology, 57(4), 364-374. doi:10.1159/000314159

Perea, M., Marcet, A., Vergara-Martinez, M., \& Gomez, P. (2016). On the Dissociation of Word/Nonword Repetition Effects in Lexical Decision: An Evidence Accumulation Account. Frontiers in Psychology, 7, 215. doi:10.3389/fpsyg.2016.00215

Pezdek, K. (1987). Memory for Pictures: A Life-Span Study of the Role of Visual Detail. Child Development 58(3), 807-815.

Polich, J. (2007). Updating P300: an integrative theory of P3a and P3b. Clinical Neuropsychology, 118(10), 2128-2148. doi:10.1016/j.clinph.2007.04.019

Polidori, C., Zen, Y.-C., Zaccheo, D., \& Amenta, F. (1993). Age-related changes in the visual cortex: a review. Archives of Gerontology and Geriatrics, 17(3), 154-164.

Poll, S., Mittag, M., Musacchio, F., Justus, L. C., Giovannetti, E. A., Steffen, J., . . . Fuhrmann, M. (2020). Memory trace interference impairs recall in a mouse model of Alzheimer's disease. Nat Neurosci, 23(8), 952-958. doi:10.1038/s41593-020-0652-4

Poppenk, J., Kohler, S., \& Moscovitch, M. (2010). Revisiting the novelty effect: when familiarity, not novelty, enhances memory. Journal of Experimental Psychology: Learning, Memory, and Cognition, 36(5), 1321-1330. doi:10.1037/a0019900

Poppenk, J., McIntosh, A. R., Craik, F. I., \& Moscovitch, M. (2010). Past experience modulates the neural mechanisms of episodic memory formation. Journal of Neuroscience, 30(13), 4707-4716. doi:10.1523/JNEUROSCI.5466-09.2010

Poppenk, J., \& Moscovitch, M. (2011). A hippocampal marker of recollection memory ability among healthy young adults: contributions of posterior and anterior segments. Neuron, 72(6), 931-937. doi:10.1016/j.neuron.2011.10.014

Rajaram, S. (1996). Perceptual effects on remembering: recollective processes in picture recognition memory. Journal of Experimental Psychology: Learning, Memory, and Cognition, 22(2), 365-377. 
Ramon, M., \& Gobbini, M. I. (2017). Familiarity matters: A review on prioritized processing of personally familiar faces. Visual Cognition, 26(3), 179-195. doi:10.1080/13506285.2017.1405134

Ranganath, C., \& Rainer, G. (2003). Neural mechanisms for detecting and remembering novel events. Nature Reviews Neuroscience, 4(3), 193-202. doi:10.1038/nrn1052

Rangel-Gomez, M., \& Meeter, M. (2016). Neurotransmitters and Novelty: A Systematic Review. J Psychopharmacol, 30(1), 3-12. doi:10.1177/0269881115612238

Ratcliff, R. (1993). Methods of dealing with reaction time outliers. Psychological Bulletin, 114, 510-532.

Ratcliff, R., Clark, S., \& Shiffrin, R. (1990). The list strength effect: I. Data and discussion. Journal of Experimental Psychology: Learning, Memory, and Cognition, 16, 163-178.

Reder, L. M., Nhouyvanisvong, A., Schunn, C. D., Ayers, M. S., Angstadt, P., \& Hiraki, K. (2000). A mechanistic account of the mirror effect for word frequency: a computational model of remember-know judgments in a continuous recognition paradigm. J Exp Psychol Learn Mem Cogn, 26(2), 294-320. doi:10.1037//0278-7393.26.2.294

Redoblado, M. A., Grayson, S. J., \& Miller, L. A. (2003). Lateralized-temporallobe-lesion effects on learning and memory: examining the contributions of stimulus novelty and presentation mode. Journal of Clinical and Experimental Neuropsychology, 25(1), 36-48. doi:10.1076/jcen.25.1.36.13625

Reynolds, G. D., Guy, M. W., \& Zhang, D. (2011). Neural Correlates of Individual Differences in Infant Visual Attention and Recognition Memory. Infancy, 16(4), 368-391. doi:10.1111/j.1532-7078.2010.00060.x

Rhodes, S., Greene, N. R., \& Naveh-Benjamin, M. (2019). Age-related differences in recall and recognition: a meta-analysis. Psychon Bull Rev, 26(5), 15291547. doi:10.3758/s13423-019-01649-y

Rugg, M. D., \& Allan, K. (2000). Event-related potential studies of memory. In E. Tulving \& F. I. M. Craik (Eds.), The Oxford handbook of memory (pp. 521537): Oxford University Press.

Rugg, M. D., \& Curran, T. (2007). Event-related potentials and recognition memory. Trends in Cognitive Sciences, 11(6), 251-257. doi:10.1016/j.tics.2007.04.004

Salthouse, T. A. (1996). The Processing-Speed Theory of Adult Age Differences in Cognition. Psychological Review, 103(3), 403-428.

Sambeth, A., Riedel, W. J., Klinkenberg, I., Kahkonen, S., \& Blokland, A. (2015). Biperiden selectively induces memory impairment in healthy 
volunteers: no interaction with citalopram. Psychopharmacology (Berl), 232(11), 1887-1897. doi:10.1007/s00213-014-3822-9

Schacter, D. L., \& Dodson, C. S. (2001). Misattribution, false recognition and the sins of memory. Philos Trans R Soc Lond B Biol Sci, 356(1413), 1385-1393. doi:10.1098/rstb.2001.0938

Schomaker, J., \& Meeter, M. (2015). Short- and long-lasting consequences of novelty, deviance and surprise on brain and cognition. Neurosci Biobehav Rev, 55, 268-279. doi:10.1016/j.neubiorev.2015.05.002

Seidenberg, M. S., Plaut, D. C., Petersen, A. S., McClelland, J. L., \& McRae, K. (1994). Nonword pronunciation and models of word recognition. Journal of Experimental Psychology: Human Perception and Performance, 20(6), 1177-1196.

Silver, H., \& Geraisy, N. (1995). Effects of Biperiden and Amantadine on Memory in Medicated Chronic Schizophrenic Patients A Double-blind Cross-over Study. British Journal of Psychiatry, 166, 241-243.

Smith, A. D., Park, D. C., Cherry, K., \& Berkovsky, K. (1990). Age differences in memory for concrete and abstract pictures 45, 205-209.

Smith, M. E., \& Guster, K. (1993). Decomposition of recognition memory eventrelated potentials yields target, repetition, and retrieval effects. Electroencephalography and clinicalNeurophysiology, 86, 335-343.

Snodgrass, J. G., \& Corwin, J. (1988). Pragmatics of measuring recognition memory: Applications to dementia and amnesia. Human experimental psychology, 117, 34-50.

Solso, R. L. (1995). Cognitive Psychology (4 ed.). Boston Allyn and Bacon.

Stanislaw, H., \& Todorow, N. (1999). Calculation of signal detection theory measures. Behavior Research Methods, Instruments, \& Computers 3, 37-149.

Sudo, Y., Suhara, T., Suzuki, K., Okubo, Y., Yoshikawa, K., Uchida, S., . . . Matsushita, M. (1998). Muscrinic receptor occupacy by biperiden inliving human brain Life Sciences, 64(8), 99-104.

Titov, N., \& Knight, R. G. (1997). Adult Age Differences in Controlled and Automatic Memory Processing. Psychol and Aging, 12(4), 565-573.

Togo, F., Lange, G., Natelson, B. H., \& Quigley, K. S. (2015). Attention network test: assessment of cognitive function in chronic fatigue syndrome. $J$ Neuropsychol, 9(1), 1-9. doi:10.1111/jnp.12030

Toth, M., Sambeth, A., \& Blokland, A. (2021). EEG Correlates of Old/New Discrimination Performance Involving Abstract Figures and NonWords. Brain Sci, 11(6). doi:10.3390/brainsci11060719

Trahan, D. E., Larrabee, G. J., \& Levin, H. S. (1986). Age-related differences in recognition memory for pictures. Experimental Aging Research, 12, 147150. 
Tulving, E., \& Kroll, N. (1995). Novelty assessment in the brain and long-term memory encoding. Psychonomic Bulletin and Review, 2(3), 387-390. doi:10.3758/BF03210977

Tulving, E., Markowitsch, H. J., Craik, F. E., Habib, R., \& Houle, S. (1996). Novelty and familiarity activations in PET studies of memory encoding and retrieval. Cerebral Cortex, 6(1), 71-79.

Tulving, E., Markowitsch, H. J., Shitij, K., Habib, R., \& Sylvain, H. (1994). Novelty encoding networks in the human brain: positron emission tomography data. NeuroReport, 5, 2525-2528.

Tulving, E., \& Schacter, D. L. (1990). Priming and human memory systems. Science, 247(4940), 301-306. doi:10.1126/science.2296719

Umanath, S., \& Marsh, E. J. (2014). Understanding How Prior Knowledge Influences Memory in Older Adults. Perspect Psychol Sci, 9(4), 408-426. doi:10.1177/1745691614535933

Uttl, B., Henry, M., \& Baltimore, K. (2007). Are smaller age differences on old/new recognition versus free recall tests artifacts of easy memory tests? . Canadian Journal of Experimental Psychology, 61, 374.

Vachon, F., Hughes, R. W., \& Jones, D. M. (2012). Broken expectations: Violation of Expectancies, Not Novelty, Captures Auditory Attention. Journal of Experimental Psychology: Learning, Memory, and Cognition 38(1), 164-177. doi:0.1037/a0025054

van Dinteren, R., Arns, M., Jongsma, M. L., \& Kessels, R. P. (2014). P300 development across the lifespan: a systematic review and metaanalysis. Plos One, 9(2), e87347. doi:10.1371/journal.pone.0087347

van Hooren, S. A., Valentijn, A. M., Bosma, H., Ponds, R. W., van Boxtel, M. P., \& Jolles, J. (2007). Cognitive functioning in healthy older adults aged 64-81: a cohort study into the effects of age, sex, and education. Neuropsychol Dev Cogn B Aging Neuropsychol Cogn, 14(1), 40-54. doi:10.1080/138255890969483

Van Petten, C., \& Senkfor, A. J. (1995). Memory for words and novel visual patterns: Repetition, recognition, and encoding effects in the eventrelated brain potential. Psychophysiology, 33(5), 491-506.

Verde, M. F., \& Rotello, C. M. (2007). Memory strength and the decision process in recognition memory. Memory and Cognition, 35(2), 254-262.

Vingerhoets, C., Bakker, G., van Dijk, J., Bloemen, O. J. N., Wang, Y., Chan, R. C. K., . . van Amelsvoort, T. (2017). The effect of the muscarinic M1 receptor antagonist biperiden on cognition in medication free subjects with psychosis. Eur Neuropsychopharmacol, 27(9), 854-864. doi:10.1016/j.euroneuro.2017.06.014 
Voss, J. L., \& Paller, K. A. (2009). Remembering and knowing: electrophysiological distinctions at encoding but not retrieval. Neuroimage, 46(1), 280-289. doi:10.1016/j.neuroimage.2009.01.048

Wammes, J. D., Meade, M. E., \& Fernandes, M. A. (2016). The drawing effect: Evidence for reliable and robust memory benefits in free recall. The Quarterly Journal of Experimental Psychology, 69(9), 1752-1776. doi:10.1080/17470218.2015.1094494

Wang, E., Zhou, G., Huang, H., Min, Q., Chao, W., \& Wang, Y. (2017). N400 and P600 Effect of Chinese Words Recognition. NeuroQuantology, 15(4). doi:10.14704/nq.2017.15.4.1172

Weisz, J., \& Czigler, I. (2006). Age and novelty: Event-related brain potentials and autonomic activity. Psychophysiology, 43(3), 261-271. doi:10.1111/j.1469-8986.2006.00395.x

Wezenberg, E., Verkes, R. J., Sabbe, B. G., Ruigt, G. S., \& Hulstijn, W. (2005). Modulation of memory and visuospatial processes by biperiden and rivastigmine in elderly healthy subjects. Psychopharmacology (Berl), 181(3), 582-594. doi:10.1007/s00213-005-0083-7

Wittmann, B. C., Bunzeck, N., Dolan, R. J., \& Duzel, E. (2007). Anticipation of novelty recruits reward system and hippocampus while promoting recollection. Neuroimage, 38(1), 194-202. doi:10.1016/j.neuroimage.2007.06.038

Wixted, J. T. (2007). Dual-process theory and signal-detection theory of recognition memory. Psychol Rev, 114(1), 152-176. doi:10.1037/0033295X.114.1.152

Wixted, J. T., \& Stretch, V. (2004). In defense of the signal detection interpretation of remember/know judgments. . Psychon. Bull. Rev., 11, 616-641.

Wolk, D. A., Sen, N. M., Chong, H., Riis, J. L., McGinnis, S. M., Holcomb, P. J., \& Daffner, K. R. (2009). ERP correlates of item recognition memory: effects of age and performance. Brain Res, 1250, 218-231. doi:10.1016/j.brainres.2008.11.014

Xie, Q., Pan, J., Chen, Y., He, Y., Ni, X., Zhang, J., . . Y Yu, R. (2018). A gazeindependent audiovisual brain-computer Interface for detecting awareness of patients with disorders of consciousness. BMC Neurol, 18(1), 144. doi:10.1186/s12883-018-1144-y

Yonelinas, A. P. (2002). The Nature of Recollection and Familiarity: A Review of 30 Years of Research. Journal of Memory and Language, 46(3), 441-517. doi:10.1006/jmla.2002.2864

Yonelinas, A. P., Kroll, N. E. A., Dobbins, I., Lazzara, M. M., \& Knight, R. T. (1998). Recollection and familiarity deficits in amnesia: Convergence of remember-know, process dissociation. Neuropsychology, 12(3), 323-339. 
Zacks, R. T., \& Hasher, L. (2006). Aging and long-term memory: Deficits are not inevitable. In E. Bialystok \& F. I. M. Craik (Eds.), Lifespan cognition: Mechanisms of change (pp. 162-177). New York: NY: Oxford University Press. doi:10.1093/acprof:oso/9780195169539

Ziegler, J. C., Besson, M., Jacobs, A. M., \& Nazir, T. A. (1995). Word Pseudoword and Nonword Processing A Multitask Comparison Using Event-Related Brain Potentials. Journal of Cognitive Neuroscience, 9(6), 758-775. 


\section{VI.7. Summary}

Recognition memory is a form of episodic memory that promotes the discrimination of new items or experiences from previously encountered, familiar ones. Therefore, it has an essential role in record-keeping and updating the information we store about the environment. As such, it can effectively guide our behavior. Nevertheless, there is considerable debate in recent theories of memory about how the brain processes and stores new and familiar information. Some researchers argue for processing benefits of the former, while others argue for the mnemonic benefits of the latter. These mixed results can be attributed to differences in the experimental designs and the type of stimuli used. Therefore, this dissertation aimed to investigate further the processes underlying old/new recognition, such as pre-experimental stimulus familiarity, memory strength, and age. Throughout this dissertation, we used a three-phase old/new recognition memory paradigm (deep memorization, shallow memorization, and recognition) to test how the brain processes and responds to pre-experimentally unfamiliar abstract figures and non-words. Memory strength was manipulated as a function of the Levels of Processing. Strong memories were induced with deep encoding and repetition, while weak memories were achieved via shallow encoding without repetition. The included experiments' results were anticipated to reveal the factors that might influence effective recognition performance of stimuli without a pre-experimentally existing memory or meaning. Knowing how the brain processes these items can significantly improve our understanding of visual, lexical, and orthographic memory processing and contribute to designing relevant aging models.

In Chapter II, the role of memory strength and pre-experimental familiarity was tested using the above-described paradigm. To account for the effects of pre-experimental familiarity, we used pre-experimentally familiar and 
unfamiliar visual and verbal materials (figures and words vs. abstract figures and non-words). The findings revealed that recognition performance primarily depends on experimentally induced memory strength as no differences were found between pre-experimental familiar and unfamiliar old item recognition accuracy. However, the new, unfamiliar figures were recognized better than the familiar ones, probably due to the more profound distinctiveness of the former. Regarding memory strength, we found that, independently of the stimulus being pre-experimentally familiar or unfamiliar, new item identification exceeded the correct old item recognition when the stimulus memory was weakly embedded but not when it was strongly embedded. Thus, this study indicates that while pre-experimental familiarity has a differential impact on visual and verbal materials, this is not the case for memory strength.

Chapter III reveals the underlying brain responses during old/new recognition of abstract figures and non-words using EEG. The results showed that successful new item identification was marked by a combination of the absence of familiarity (N400) and recollection (P600). For both the abstract figures and the non-words, the parietal P600 differentiated between the old and new items (late old/new effects). This study extends current knowledge on the processing of pre-experimentally unfamiliar visual and verbal items by showing that their discrimination depends on experimentally induced memory strength and that the underlying brain processes differ. Nevertheless, the P600, similarly to pre-experimentally familiar figures and words, likely reflects improved recognition memory of meaningless visual and verbal items.

In Chapter IV, the above-described paradigm was tested in healthy young and elderly volunteers. It was found that older adults relative to the young showed impairment in the correct identification of new items. As indicated by the lower discriminability indexes, the elderly also had difficulties discriminating the strongly (drawn/semantically processed) and the weakly (studied) embedded 
abstract figures but not the non-words. Age-related differences in reaction times were also only evident with the abstract figures. Finally, our results revealed that the recognition performance was equally affected by memory strength in both age groups. The current findings agree with previous research on age-related impairment in new item recognition, which can be attributed to misrecollection, decreased sensitivity to novelty, and less accurate novelty assessment in the elderly than the young. The detected age effects on the discriminability of the drawn and studied abstract figures agree with the age-related impairment in the perceptual encoding hypothesis and support the notion related to the need for environmental support to reduce age effects. The lack of age effects during the processing of the non-words indicates that age effects on discriminability appear to be stimulus-dependent. Thus, the current results support the notion that recognition memory in aging is only impaired under certain conditions and likely depends on the stimuli used.

The study in Chapter $V$ investigated the effects of the muscarinic type 1 antagonist, biperiden, using the paradigm mentioned above with the aim to reveal whether this drug can be effective in modeling age-related memory impairments. The results of a double-blind, placebo-controlled, 2-way cross-over study are presented. It was found that $4 \mathrm{mg}$ biperiden impaired recognition accuracy and prolonged reaction times of the drawn and the studied abstract figures. However, participants were biased towards 'old' responses in the placebo condition. The recognition of the new abstract figures was unaffected by the drug. Biperiden did not affect the recognition of the non-words. It was concluded that although biperiden may model age-related deficits in episodic memory, the current findings indicate that biperiden cannot mimic age-related deficits in recognition performance involving abstract figures and non-words. 
Chapter VI includes the general discussion and reflections on the main findings of this dissertation, including the implications and directions for future research. Based on consistent findings presented in this dissertation, it can be said that the paradigm has reliably and consistently shown throughout all presented experiments the benefits of deeper Levels of Processing and repetition over shallow encoding without repetition. Additionally, experimentally induced memory strength has been found to affect old/new recognition. Namely, items with weak memory were less well recognized than the new ones. In contrast, items having strong memory representations were recognized better or just as well as the new ones. Furthermore, these findings were independent of preexperimental familiarity, age, and pharmacological treatment with BIP, which suggests that experimentally induced memory strength is a stable and vital factor influencing old/new recognition. Regarding the underlying brain processes, the impact of age, and the blocking of the antimuscarinic receptors, it seems that there is a marked difference in the processing of pre-experimentally unfamiliar visual and verbal items. Therefore, further studies might benefit from including stimuli with both modalities to account for more accurate findings, especially considering the aging brain. 


\section{VI.8. Impact Paragraph}

The ability to respond to distinct, unusual, or new stimuli is an instinct that drives the unconscious sensory-based and continuous exploration. Thus, from an evolutionary perspective identifying something as new is essential for learning, adapting to changes, and survival. According to research, newborns show an orienting response, as they automatically direct their attention to stimulus novelty. However, evidence also shows that infants do not always favor new over old things. Such old-new shifts remain as we get older and wiser. For example, we are naturally drawn to anything outside the status quo. Also, we often fancy the new at the expense of the old. Other times, we stick to old run-in routines, which offer convenience and stability. Despite decades of research, it remains unknown and highly debated which type of information, old or new, is preferentially processed by the brain. This is especially true for stimuli that have likely not been seen outside an experimental context.

Considering the above, the main objective in the current work was to investigate the behavioral and electrophysiological processes underlying old/new recognition of pre-experimentally unfamiliar abstract figures and nonwords in a set of five experiments. Specifically, the goal was to examine the role of memory strength and age in such processing. Memory and novelty detection have both previously been shown to rely on the cholinergic system of the brain. The cholinergic system comprises nerve cells, such as muscarinic and nicotinic receptors, that use the neurotransmitter acetylcholine when communicating with each other. Aging is associated with deficits in cholinergic neurotransmission. Thus, we also tested whether cholinergic modulation with the muscarinic type 1 receptor antagonist drug, biperiden, could pharmacologically model the memory impairment of healthy elderly. To find out the answers to these questions, we used a memory paradigm with abstract figures and non-words. First, the 
participants were asked to redraw the abstract figures and mention existing rhyming words for the non-words. Afterward, they had to memorize the presented items without any additional tasks. Here, the items from the first phase were repeated and mixed with some new ones. Finally, they were shown the before seen, old items mixed with some previously not presented ones. Their task was to decide if the presented stimulus was familiar (old) or not (new). Repetition and the drawing/rhyming task created strong memories, while simply memorizing the items led to weak memory formation. This way, we could manipulate the strength of the different item memories. Both healthy young and old adults performed the tests.

Every experiment has shown that people were better at recognizing something as 'new' than 'old'. However, this was only true when the familiar items relied on weak memories. In contrast, when the familiar stimuli were memorized via drawing or rhyming words, they could be recognized as 'old' just as good as the new items were identified as 'new'. These findings were consistent in the young, elderly, and when we used a pharmacological manipulation. It also did not matter whether the items were pre-experimentally familiar words and figures or were utterly new and likely never before seen abstract figures and nonwords. Therefore, we concluded that a particular memory's strength plays a vital role in how well one can distinguish new from old. Furthermore, the behavior and the brain activity were different for the different stimuli. For example, when the brain distinguishes between the new and the weakly embedded abstract figures, the event-related potentials associated with familiarity (FN400) and recollection (P600) are missing for the weak memories. On the other hand, when the brain accurately recognized the strongly embedded non-words, there was an increase in the memory-related P600 component compared to the studied and new items. 
Concerning the impact of healthy aging, we found that the seniors were impaired in identifying the new stimuli correctly and had difficulties with discriminating the old abstract figures. However, age did not affect the processing of the non-words. Thus, these findings indicate that processing problems in aging can differ for visual and verbal stimuli. In contrast to the expectations, the biperiden-induced effects showed the opposite pattern. Namely, the drug impaired old item recognition while leaving correct identification of the new stimuli unaffected. Thus, it can be said that biperiden could not adequately mimic the memory deficits of the healthy elderly.

The presented results clarify the dispute on how the brain discriminates old information from new and how aging affects such processing. These findings extend current knowledge on how we process and respond to pre-experimentally unfamiliar abstract figures and non-words. Abstract figures and non-words hardly involve semantics, thus verbalizing what we see or experience. Therefore, knowing how the brain processes these items can improve our understanding of visual, lexical, and orthographic memory processing and contribute to establishing relevant aging models. Furthermore, the current findings are useful for addressing how novel and familiar information can be effectively presented in recognition memory paradigms. The presented findings can also explain why, in some situations, old information is less accurately processed than new. The stakeholders for this aspect are a broad spectrum of clinical and non-clinical psychologists.

Moreover, the effects of aging on new item identification show a possible direction for developing new drugs that could improve somebody's cognitive performance. It seems wise to consider other drugs that, for instance, target the brain's dopamine system to improve our ability to react to new information. Such assumptions make sense in light of findings showing that stimulus novelty engages the brain's dopamine-mediated reward centers. Nevertheless, this bears 
future research. It could also benefit patients with brain disorders such as schizophrenia, dementia, or Alzheimer's disease. Namely, in these conditions, next to the fact that memory is impaired, both the cholinergic and the dopaminergic brain systems play an essential role. Thus, the results could shed better light on the cognitive impairments these patients face, which could ultimately provide improved means for adequate diagnosis and treatment options. It is noteworthy mentioning that life quality is a crucial factor in general and psychological well-being, especially with increasing age. Also, the number of demented patients is expected to increase substantially in the coming decades, putting much pressure on the healthcare system and the caregivers. 


\section{VI.9. Acknowledgements}

Becoming a PhD was a rockier road than anticipated, with many ups and downs. Therefore, I am incredibly thankful to my supervisors, Prof. dr. Arjan Blokland and Dr. Anke Sambeth and my colleague, Nadia Hutten. Their contribution, support, academic guidance, friendship, encouragement, and competence concerning this dissertation's content and the included research are highly appreciated. I also wish to thank the students who contributed to the projects in this dissertation, including Gayel Duran, Sandra Casanova Gallinato, and Joris van Bohemen. Furthermore, I wish to thank the members of the Assessment Committee, Prof. dr. Wim Riedel, Prof. dr. Martijn Meeter, Prof. dr. Leon Kenemans and Dr. Conny Quaedflieg. Their constructive feedback and critical approach have undoubtedly contributed to my professional development. Finally, I am very grateful to my parents, Dr. Mária Tóth and Dr. László Tóth, my partner Joeri van Tongeren, my friend Balázs Borsodi, who have supported me through these challenging times. 


\section{VI.10. Curriculum Vitae}

Mónika Tóth was born on November 25th, 1979, in Beregovo (Soviet Union). She completed her secondary education at the Zalka Máté Gimnázium, in Fehérgyarmat (Hungary). In 1998, she moved to Budapest (Hungary) to study Economics at the Budapest University of Business Administration and Management. She obtained her Master's degree in Business and Administration with a specialization in Marketing and Communication, in 2003. Afterward, she worked as an economist and international business specialist in the healthcare and pharma industries. In 2012, she moved to the Netherlands, Maastricht.

In 2013, she decided to follow her dreams and started her Bachelor's studies in Psychology at Maastricht University. She became a research assistant to Dr. Anke Sambeth in 2015. In 2016, after graduating as a psychologist, she started the Research Master program in Cognitive and Clinical Neuroscience with the specialization in Drug Development and Neurohealth. As part of the Master's internship, she conducted her behavioral and electrophysiological research on the effects of the antimuscarinic agent, biperiden, on the processing of abstract figures and non-words under the supervision of Dr. Sambeth and Prof. dr. Arjan Blokland.

After graduation in 2018, she started her PhD studies under the supervision of Dr. Sambeth and Prof. dr. Blokland at the department of Neuropsychology and Psychopharmacology of Maastricht University. During her PhD trajectory, she performed and designed different experiments to investigate the processing of novelty and familiarity in the aging brain. Aside from research activities, she also taught numerous Bachelor's and Master's courses at Maastricht University. 
